\title{
Linear Failure Criteria with Three Principal Stresses
}

\author{
A THESIS \\ SUBMITTED TO THE FACULTY OF THE GRADUATE SCHOOL \\ OF THE UNIVERSITY OF MINNESOTA \\ BY
}

\section{James Patrick Meyer}
IN PARTIAL FULFILLMENT OF THE REQUIREMENTS FOR THE DEGREE OF MASTER OF SCIENCE

Professor Joseph F. Labuz

March 2012 
(C) Copyright by James Patrick Meyer, 2012 


\section{Abstract}

Any failure criterion can be represented as a surface in principal stress space, and the shape of the surface depends on the functional form of the criterion. For isotropic materials that exhibit a pressure dependence on strength, the simplest failure criterion is a linear function, and the failure surface is a hexagonal pyramid with a common vertex $V_{o}$ on the tension side of the hydrostatic axis. An example of a pyramidal failure surface for rock is the popular Mohr-Coulomb criterion, which is independent of the intermediate principal stress and thus contains two material parameters.

A linear failure criteria in three principal stress is formulated with three material constants: internal friction angles for (i) compression $\phi_{c}$ and (ii) extension $\phi_{e}$, and (iii) a common vertex $V_{o}$. Nonlinearity on the failure surface can be approximated by additional planes with appropriate material parameters (i), (ii), and (iii). To demonstrate the utility of the linear failure criterion, a series of conventional triaxial compression $\left(\sigma_{\|}=\sigma_{\| I}\right)$ and extension $\left(\sigma_{\|}=\sigma_{\|}\right)$experiments were performed on an isotropic rock. The results were processed using the developed data fitting techniques, and the material parameters for the six-sided pyramidal failure surface were determined. Multi-axial $\left(\sigma_{\mid} \neq \sigma_{\|} \neq \sigma_{\| I}\right)$ experiments were also performed to evaluate the nonlinearity, and a twelve-sided pyramid was constructed and the appropriate equations were derived. 


\section{Table of Contents}

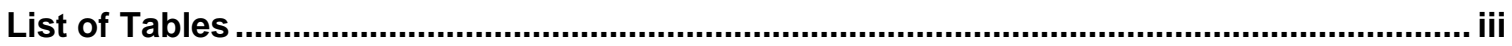

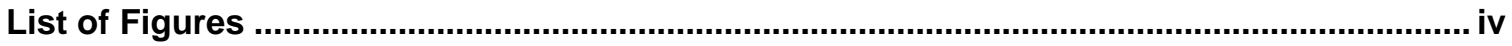

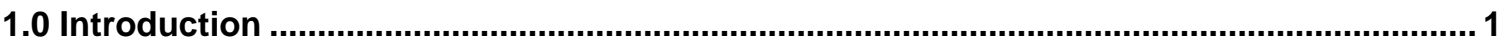

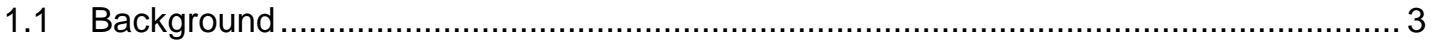

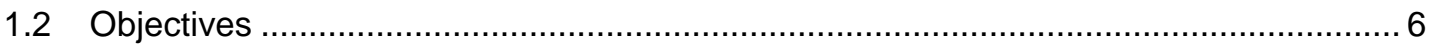

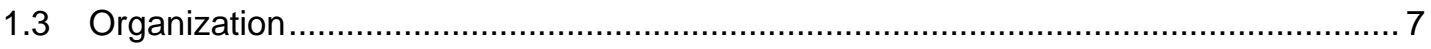

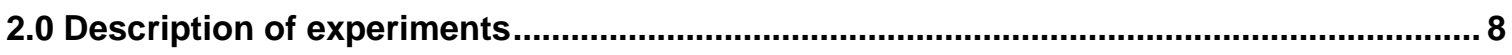

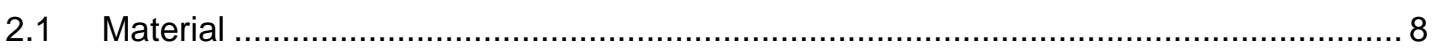

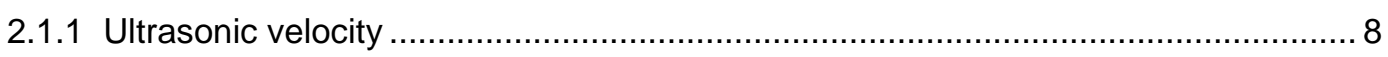

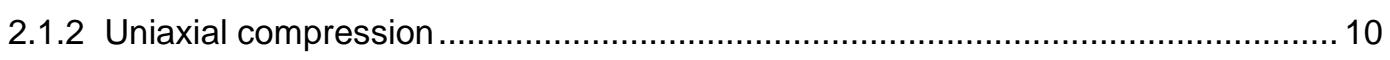

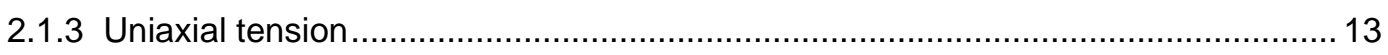

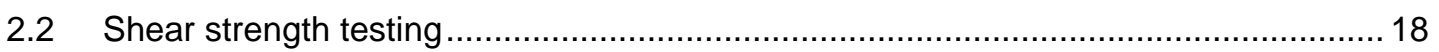

2.2.1 Conventional triaxial compression and extension.................................................... 18

2.2.2 Plane-strain compression .......................................................................... 20

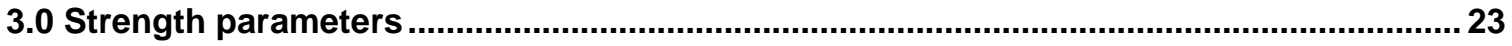

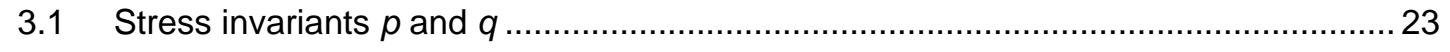

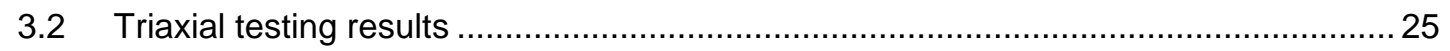

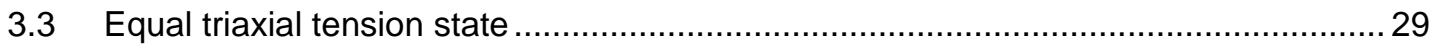

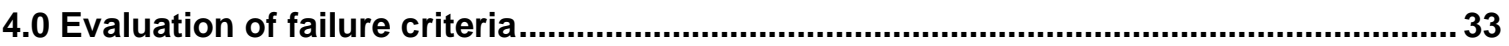

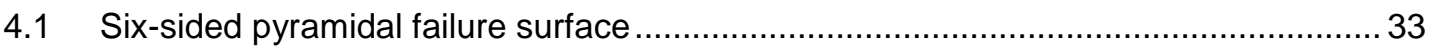

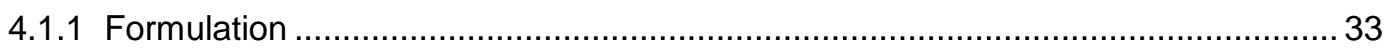

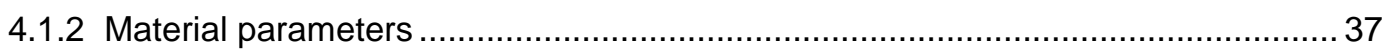

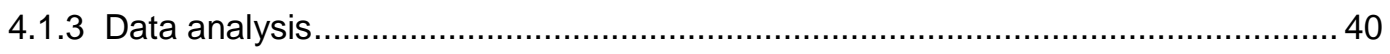

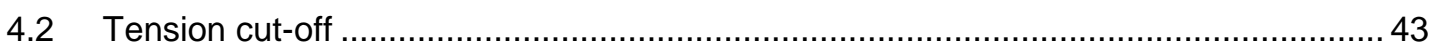

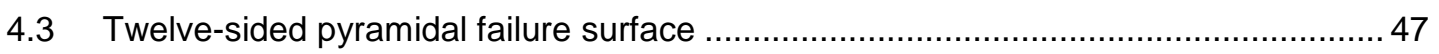

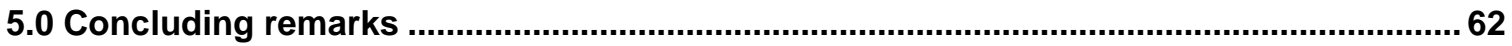

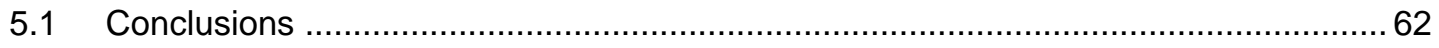

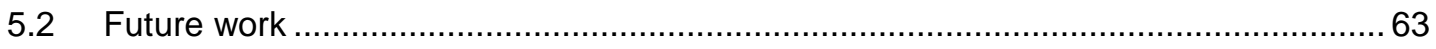

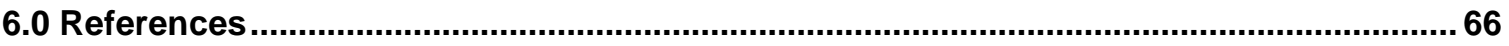




\section{List of Tables}

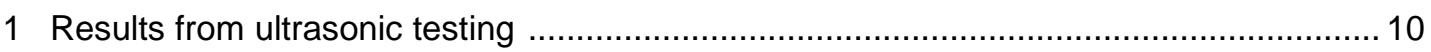

2 Uniaxial compression results of cylindrical Calcarenite specimens ................................. 11

3 Comparison of ultrasonic and uniaxial compression testing results ............................... 13

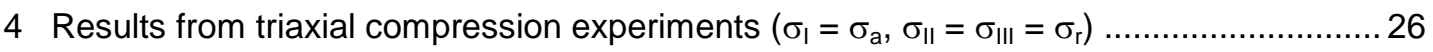

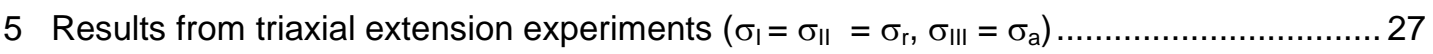

6 Strength parameters with common intercept $V_{o}$ (adjusted) and raw (unadjusted) data .. 32

7 Triaxial compression and extension results in terms of principal stresses $\sigma_{1}, \sigma_{2}$, and $\sigma_{3}$

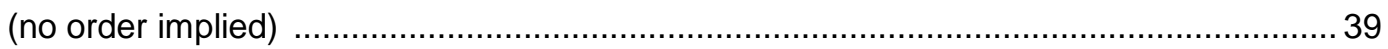

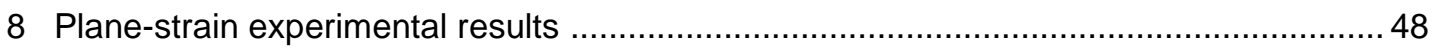

9 Material parameters determined from three different pyramidal failure surfaces ............61 


\section{List of Figures}

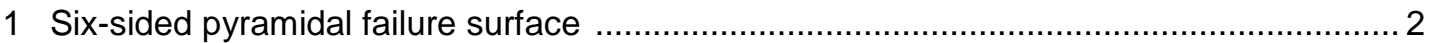

2 Piecewise linear surface approximately describing the actual response .......................... 3

3 Mohr-Coulomb failure envelope on a Mohr diagram .................................................. 4

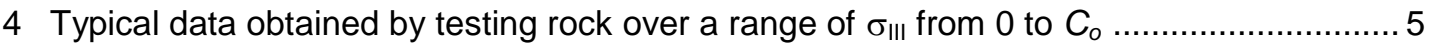

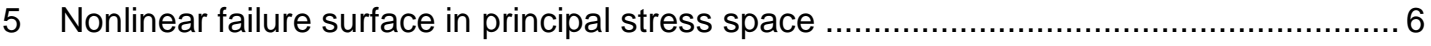

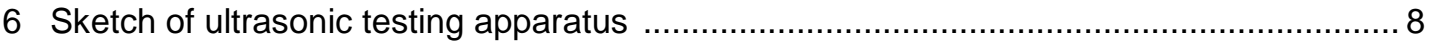

7 Mechanical response from a uniaxial compression test performed on the

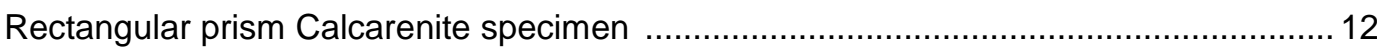

8 Deformation response from a uniaxial compression test performed on the rectangular prism Calcarenite specimen ................................................................ 13

9 Dimensions of Calcarenite specimen for uniaxial tension testing ..................................... 14

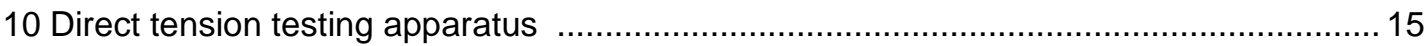

11 Axial stress - axial strain from two strain gages attached on two sides of the Calcarenite specimen located in the reduced cross-section ....................................................... 17

12 Direct tension specimen (a) before testing; (b) after testing ......................................... 18

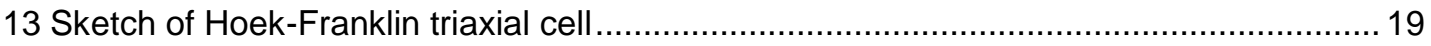

14 Sketch of UMN Plane-Strain Apparatus. (a) Elevation view. (b) Plan view ......................2 21

15 Biaxial test specimen with large brass tab exposed and AE sensor attached .................. 22

16 Failure surface described by compression and extension results ................................... 24

17 (a) Specimen influenced by a weak plane; (b) Typical compression failure plane ........... 26

18 (a) Specimen influenced by a weak plane; (b) Typical extension failure plane ................ 27

19 Triaxial compression and extension results presented in $p-q$ form ................................ 28

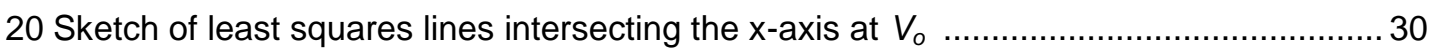

21 Triaxial compression and extension data with a common vertex .................................... 31

22 Cross-section of failure surface along hydrostatic axis corresponding to $\sigma_{2}=\sigma_{3} \ldots \ldots \ldots \ldots . . .34$

23 (a) $\pi$-plane cross-section at $\sigma_{1}=\sigma_{2}=\sigma_{3}=0$ plane, also known as the deviator

plane (b) Pyramidal surface in principal stress space ................................................... 36

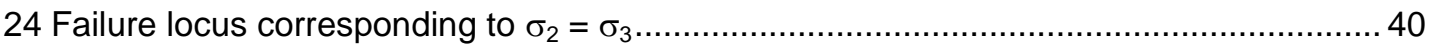

25 Cross section of MC failure surfaces and linear failure surface with

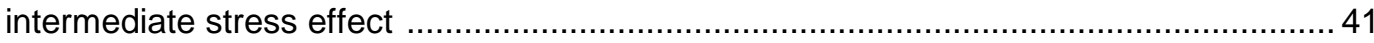

26 Experimental data points plotted on pyramidal failure surface .................................... 42 
$27 \mathrm{MC}$ failure envelope in principal stress space, with solid line representing linear failure surface with intermediate stress effect.

28 Cross-section of failure surface along hydrostatic axis corresponding to $\sigma_{2}=\sigma_{3}$ with

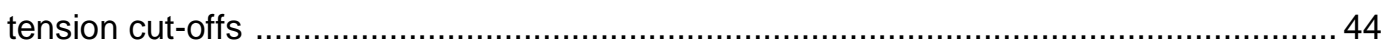

29 Pyramidal failure surface in principal stress space with tension cut-offs

30 Cross-section of failure surface along hydrostatic axis corresponding to $\sigma_{2}=\sigma_{3}$ with tension cut-offs

31 Pyramidal failure surface in principal stress space with tension cut-offs 47

32 Equipressure plane of $p=11.2 \mathrm{MPa}$ with plane-strain data point .48

33 Section showing nonlinear, piecewise linear, and linear failure surfaces 49

34 Cross-section of equipressure plane of a twelve-sided piecewise linear failure surface at $p=11.2 \mathrm{MPa}$ 50

35 Cross-section of failure surface along hydrostatic axis corresponding to $\sigma_{2}=\sigma_{3}$ 51

36 Twelve-sided pyramidal failure surface in principal stress space with theoretical failure surface 52

37 Section of an equipressure plane plotted in the $\sigma_{F}-\sigma_{E}$ coordinate system 54

38 Cross-section of failure surface, developed assuming two vertices, along hydrostatic axis corresponding to $\sigma_{2}=\sigma_{3}$ .58

39 Twelve-sided pyramidal failure surface with two vertices 59

40 Twelve-sided failure surface with tension cut-offs 61

41 Modified conventional triaxial testing apparatus 64

42 Modified UMN plane-strain apparatus 65 


\subsection{Introduction}

Design of a structure composed of or in rock, such as a slope or tunnel, requires a description of material failure that should be verified from experimental results of appropriate strength tests, where a state of stress $\sigma_{\mathrm{ij}}$ at failure can be expressed in terms of three principal stresses $\sigma_{l} \geq \sigma_{\|} \geq$ $\sigma_{\text {III }}$ (or $\sigma_{1}, \sigma_{2}, \sigma_{3}$ with no regard to order) and three directions. If the rock is isotropic, then the directional dependence disappears and a failure criterion can always be written as

$f\left(\sigma_{1}, \sigma_{2}, \sigma_{3}\right)=0$

Furthermore, any failure criterion can be represented as a surface in principal stress space (cartesian coordinates $\sigma_{1}, \sigma_{2}, \sigma_{3}$ ), and the shape of the surface depends on the form of equation (1); e.g. a linear function maps as a plane and a nonlinear function as a curvilinear surface. Because there are six possible orderings of the principal stresses, equation (1) is actually six failure surfaces, each corresponding to a particular order of principal stresses, and an example for a pressure-dependent material is a pyramidal failure surface with a common vertex $V_{o}$ (Figure 1). On a plane perpendicular to the hydrostatic axis $\left(\sigma_{1}=\sigma_{2}=\sigma_{3}\right)$ called the equipressure plane or $\pi$-plane described by

$\sigma_{1}+\sigma_{2}+\sigma_{3}=$ constant

the failure surface displays a three-fold symmetry (Figure 1), where the axes $\sigma_{1}{ }^{\prime}, \sigma_{2}{ }^{\prime}, \sigma_{3}{ }^{\prime}$ are the projections of the coordinate axes on the $\pi$-plane. Thus, any one of the $60^{\circ}$ sections uniquely defines the failure surface, a direct requirement of isotropic response.

The literature is full of various forms of the general relationship, but as suggested in the seminal paper by Paul [1968 a], the focus of this research will be limited to linear forms of equation (1):

$A \sigma_{1}+B \sigma_{2}+C \sigma_{3}=1$

With $B=0$, the linear equation represents the popular Mohr-Coulomb failure criterion and the two material constant $A$ and $C$ are related to the internal friction angle $\phi$ and the uniaxial compression strength $C_{o}$ or the vertex $V_{o}$ on the tension side of the hydrostatic axis (Figure 1). 


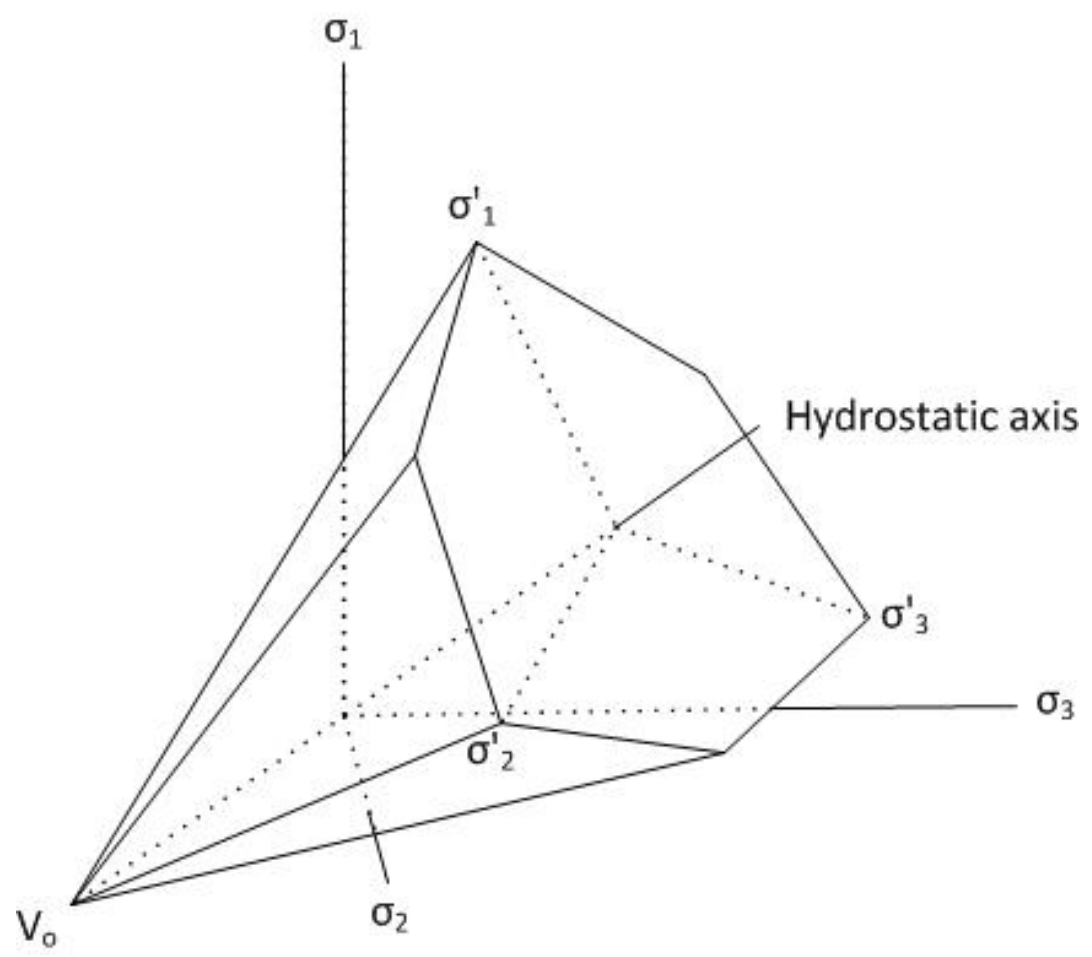

Figure 1. Six-sided pyramidal failure surface.

If all three principal stresses are admitted, then three material parameters are required, and it will be shown that $A, B$ and $C$ can be related to internal friction angles (i) $\phi_{C}$ for compression $\left(\sigma_{\|}=\sigma_{\| I I}\right)$ and (ii) $\phi_{\mathrm{e}}$ for extension $\left(\sigma_{\mathrm{l}}=\sigma_{\|}\right)$, and (iii) $V_{o}$. Note that the nonlinear response of the rock, as represented in the $\pi$-plane, can be reasonably captured by piecewise linear segments (Figure 2). 


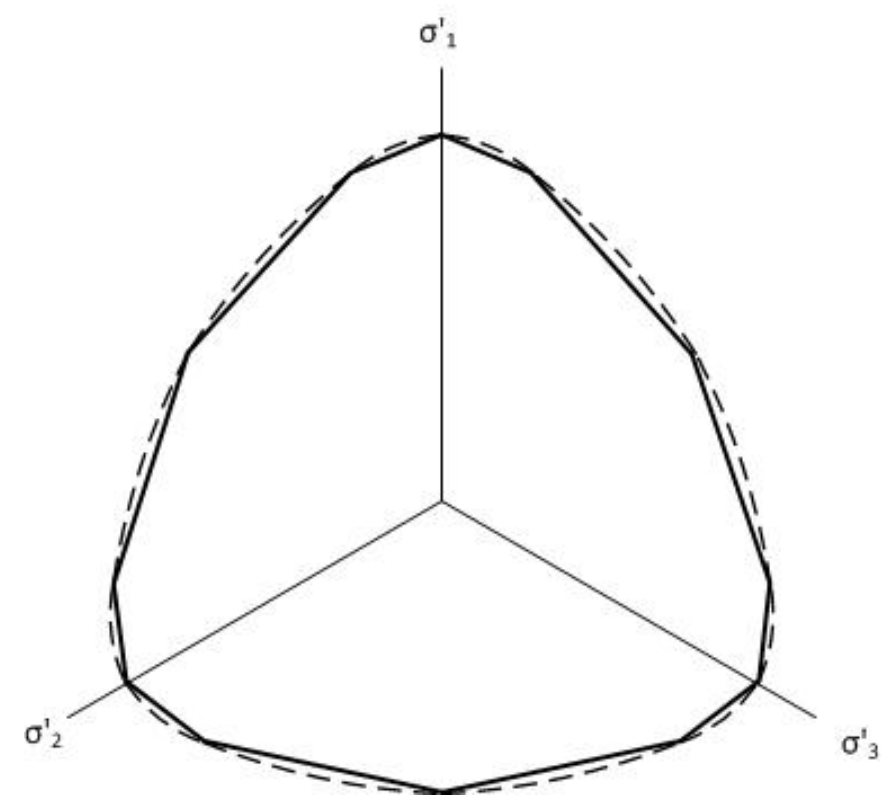

Figure 2. Piecewise linear surface (solid lines) approximately describing the actual response (nonlinear surface in dashed lines).

\subsection{Background}

\section{2}

The Mohr-Coulomb (MC) criterion is one of the most widely used theories for describing the failure of rock. This is due to a number of reasons, first of which is its mathematical simplicity. Another reason is the physical meaning of the material parameters, and lastly there is a general level of acceptance for the criterion. The MC theory can be thought of as a set of linear equations in principal stress space that represent a shear failure surface for an isotropic material, with no effect from intermediate principal stress $\left(\sigma_{\|}\right)$. However, various researchers have performed multi-axial testing, and an intermediate stress effect sometimes appears [c.f. Mogi 1967].

The Coulomb [1776] failure criterion can be written in terms of normal and shear stresses, $\sigma$ and $\tau$, on the failure plane:

$|\tau|=c+\sigma \tan \varphi$

where $c$ is cohesion and $\phi$ is the angle of internal friction. Mohr's [1900] failure hypothesis can be represented by a general failure envelope: 
$|\tau|=f(\sigma)$

Of course, the two envelopes coincide when the Mohr criterion is a linear function, which is commonly known as the MC failure theory. A representation of the $\mathrm{MC}$ failure envelope on a Mohr diagram is shown in Figure 3. The point $(\sigma, \tau)_{\mathrm{f}}$ corresponds to stresses acting on a plane with normal inclined to the major principal stress at an angle $\alpha$. The dashed circle with diameter $C_{o}$ represents the state of stress for uniaxial compression testing, with $C_{o}=$ uniaxial compressive strength (UCS). The dashed circle with diameter $T_{0}$ represents the tensile strength predicted from MC. However, this value is generally much larger than what is observed from actual testing. For example, with $\varphi=30^{\circ}, C_{o} / T_{o}=3$, while from experiments the tensile strength $\mathrm{T}$ is approximately $1 / 10$ of $C_{o}$. The discrepancy is due to the specimen failing in a tensile mode instead of the shear failure mode that MC predicts. To account for tensile failure, Paul [1961] introduced the concept of tension cut-offs.

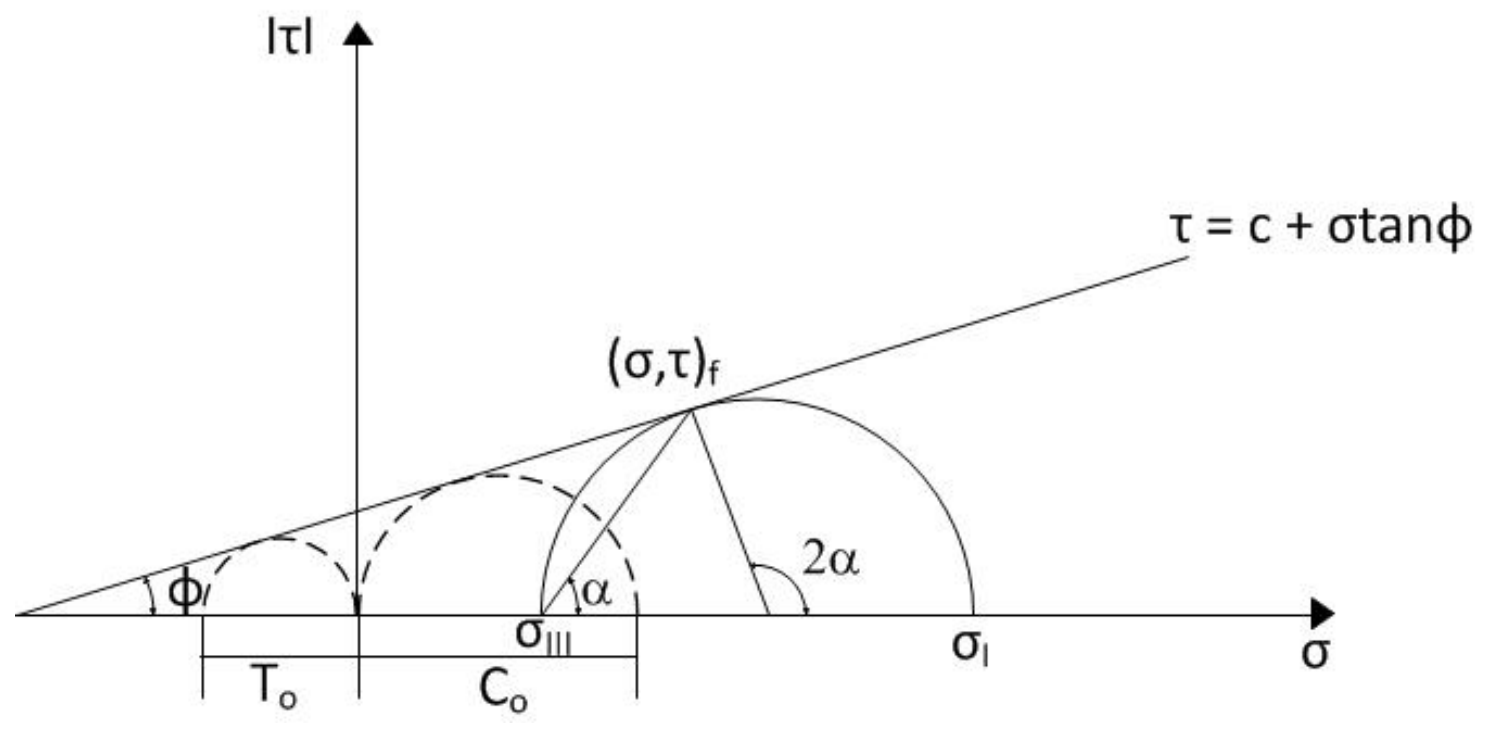

Figure 3. Mohr-Coulomb failure envelope on a Mohr diagram.

It is often convenient to represent the MC failure criterion in terms of principal stresses:

$$
\left(\sigma_{I}-\sigma_{I I I}\right)=\left(\sigma_{I}+\sigma_{I I I}\right) \sin \varphi+2 \operatorname{ccos} \varphi
$$

or

$$
\sigma_{I}=\sigma_{I I I} K_{p}+C_{o}
$$


where $\sigma_{I}=$ major principal stress, $\sigma_{\| I I}=$ minor principal stress

$K_{p}=\frac{(1+\sin \varphi)}{(1-\sin \varphi)}$

$C_{o}=2 c \frac{\cos \varphi}{1-\sin \varphi}$

It is important to note that a nonlinear failure envelope is needed to describe the behavior exhibited by many rock. Figure 4 shows typical experimental data obtained over a range of $\sigma_{\| I I}: 0$ $<\sigma_{\text {III }}<C_{0}$. Usually a nonlinear failure curve fits the data better than a straight line over the entire range of stress states. However, by considering a limited range of $\sigma_{\text {IIII }}$, a straight line provides a reasonable description of material response.

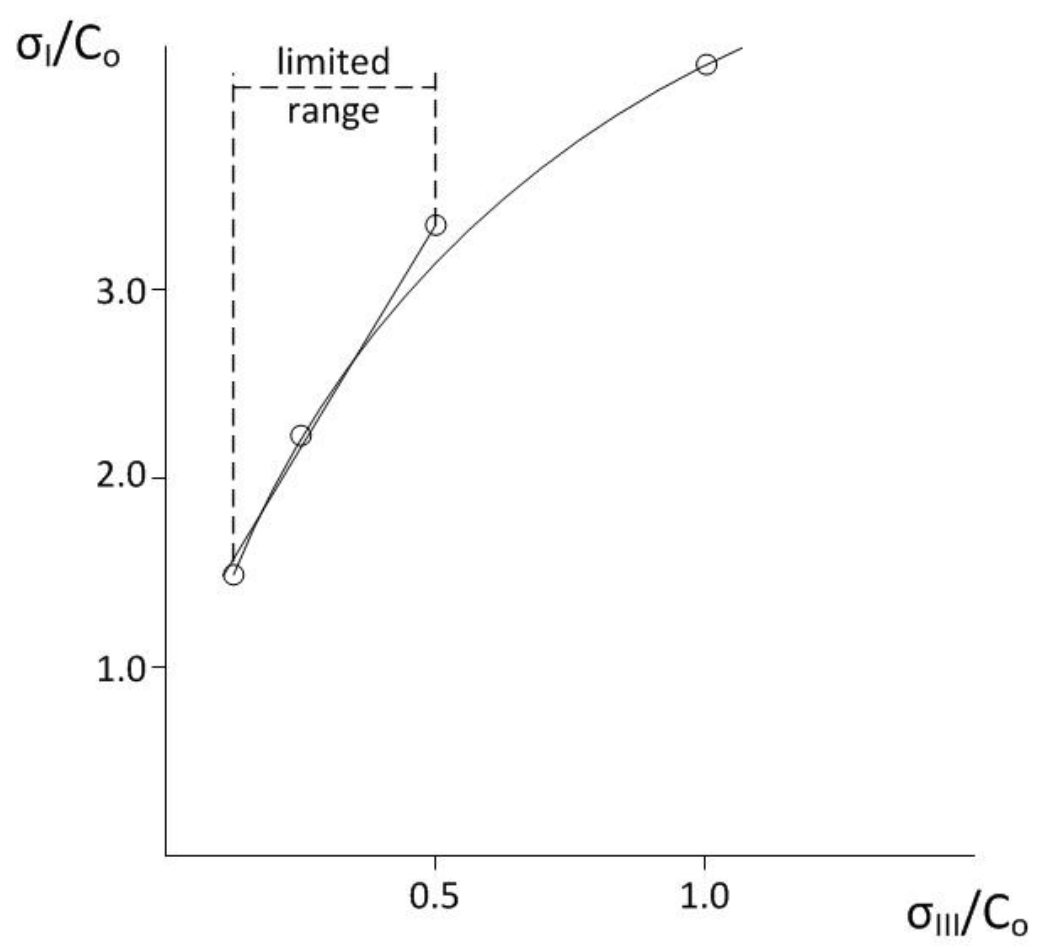

Figure 4. Typical data obtained by testing rock over a range of $\sigma_{\text {III }}$ from 0 to $C_{o}$.

The nonlinear envelope of Mohr was promoted by Hoek and Brown [1980], using a parabolic equation, which had been used as early as 1936 to describe the failure of concrete [Hoek 2002]. Mogi [1967] also suggested a nonlinear failure criterion that takes into account intermediate 
stress. Furthermore, it is recognized that the failure surface appears to be nonlinear in the $\pi$ plane (Figure 5), and a smooth surface - no corners- has advantages in numerical analyses [Borja 2003; Matsuoka and Nakai 1974; Willam and Warnke 1974]. However, sufficient experimental data is often not available to accurately describe the nonlinear surface, and the material parameters sometimes have no physical meaning.

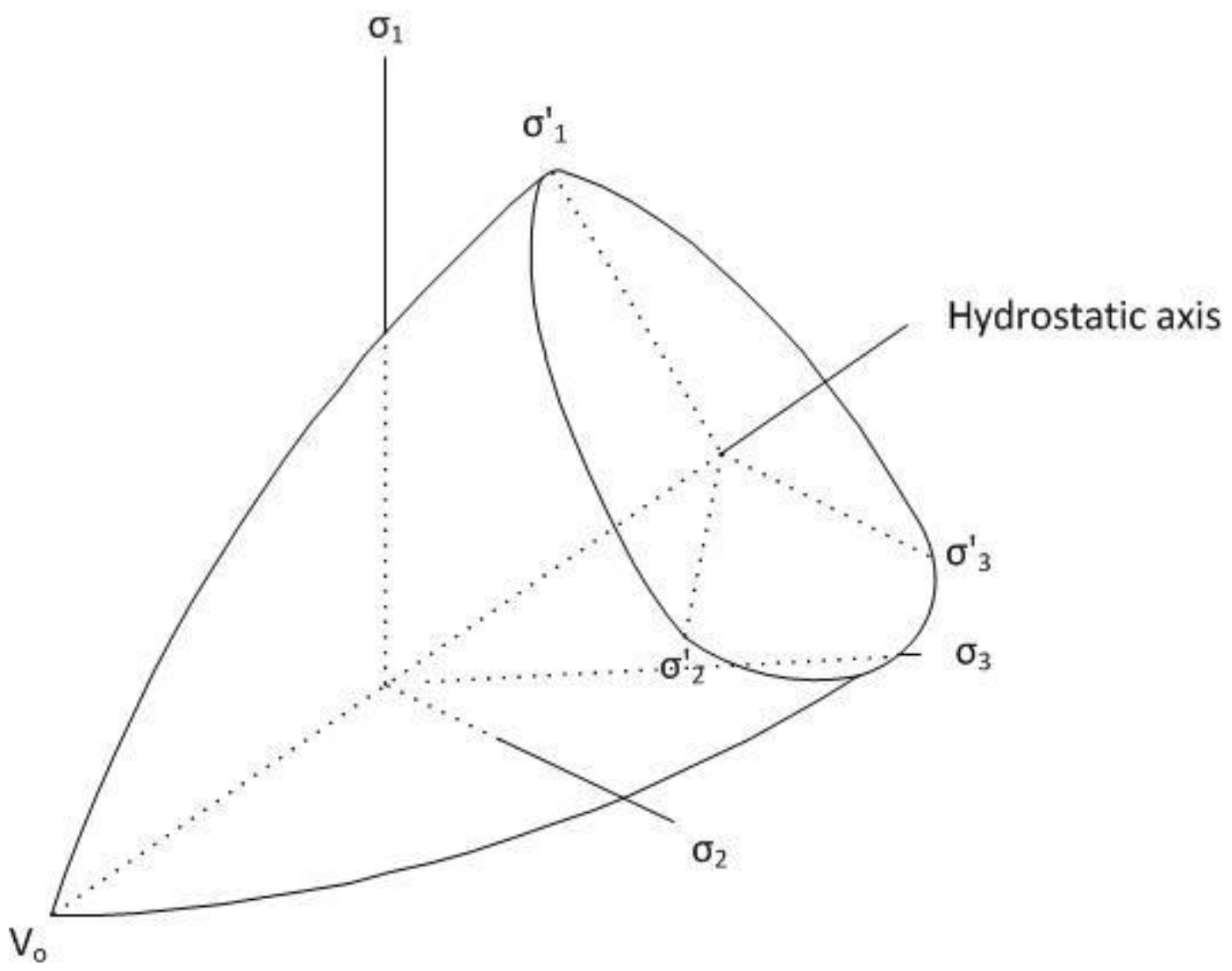

Figure 5. Nonlinear failure surface in principal stress space.

\subsection{Objectives}

The thesis presents an experimental program and analytical formulations to establish linear failure criteria for rock. From conventional triaxial testing with a Hoek-Franklin cell or similar equipment, both compression and extension testing is necessary to define a six-sided surface, which can be constructed from the three material parameters: (i) $\phi_{\mathrm{c}}$ measured from compression 
failure, (ii) $\phi_{\mathrm{e}}$ measured from extension failure, and (iii) $\mathrm{V}_{\mathrm{o}}$ calculated from data fitting. Additional testing with multi-axial loading $\left(\sigma_{1} \neq \sigma_{2} \neq \sigma_{3}\right)$ is required to enhance the failure surface, and an example of a twelve-sided pyramid with necessary parameter identification is highlighted.

\subsection{Organization}

Chapter 2 presents material parameters obtain from ultrasonic and mechanical testing, as well as detailed descriptions of the testing techniques used. Strength parameters of the material are discussed in Chapter 3, including the angles of internal friction in compression and extension, as well as cohesion values. The formulations of the six-sided and twelve-sided failure surfaces are presented in Chapter 4. Finally, a discussion of results and future work is found in Chapter 5. 


\subsection{Description of experiments}

\subsection{Material}

The rock tested was Calcarenite, a calcareous sedimentary rock formed of detritus particles ranging in diameter from $0.06-2 \mathrm{~mm}$. A single block $300 \times 300 \times 150 \mathrm{~mm}$, with density $\rho=1610$ $\mathrm{kg} / \mathrm{m}^{3}$ and porosity $\mathrm{n}=31 \%$, was used to fabricate all the specimens. The elastic parameters were determined using both ultrasonic and mechanical testing techniques. P- and S-wave velocities, along with density, provide a convenient method to determine elastic constants and degree of anisotropy. Uniaxial compression and tension specimens were instrumented with strain gages to allow for the measurement of Young's modulus $(E)$ and Poisson's ratio $(v)$.

\subsubsection{Ultrasonic velocity testing}

The ultrasonic velocity testing followed ASTM D 2845-08 standards, and the apparatus is shown in Figure 6. With appropriate transducers (Panametrics-NDT), both P- and S-waves can be generated. A Velonex high power pulse generator is used to send an electrical signal to the transmitting sensor and the digital oscilloscope when the pulse generator is activated. The signal sent to the oscilloscope acts as a trigger for the recording in the oscilloscope workstation. The electrical signal sent to the transmitter is converted into a mechanical disturbance that propagates through the specimen. The receiver that is connected to the other end of the specimen converts the mechanical disturbance back into an electrical signal, which is amplified and recorded.

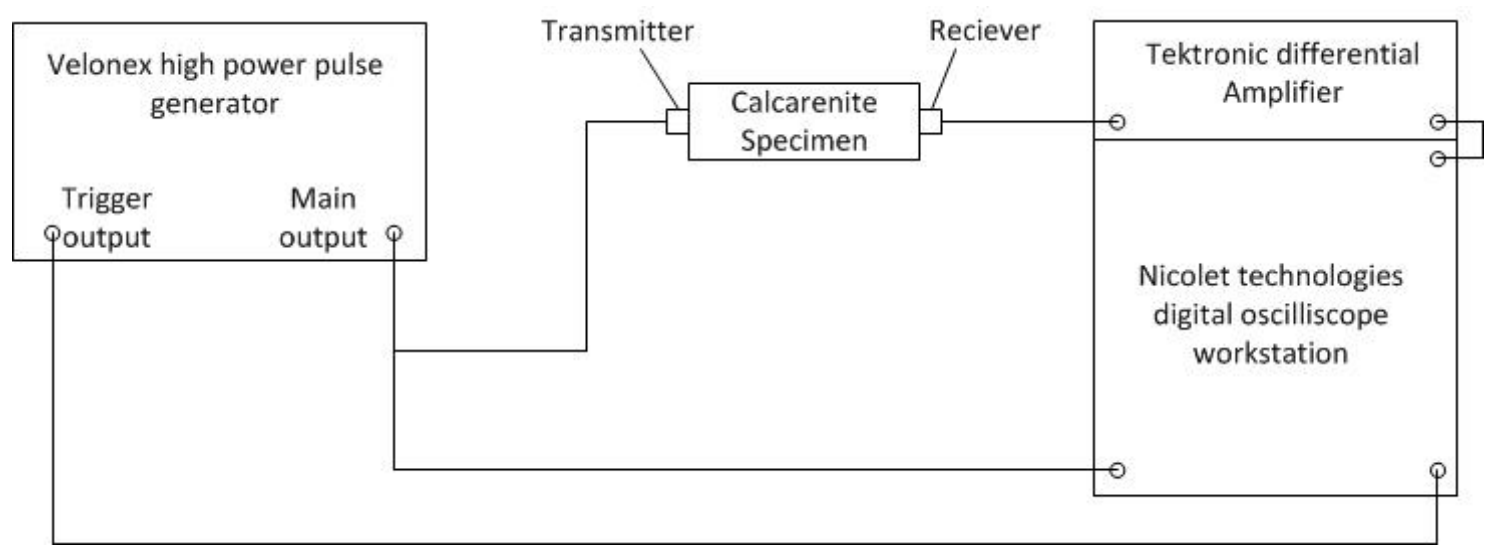

Figure 6. Sketch of ultrasonic testing apparatus. 
By visually examining the waveform, the arrival time can be determined for each wave type. This is important, as the time needed to travel through the specimen is used to determine the wave speed:

$c_{p}=\frac{l}{t_{p}}$

$c_{s}=\frac{l}{t_{s}}$

where, $l=$ length of specimen $[\mathrm{m}]$ and $t_{i}=$ first arrival $[s]$ of $\mathrm{P}$ - or S-wave

With this information and the density $\rho$, the elastic parameters can be determined using

$E=\frac{\rho c_{s}^{2}\left(3 c_{p}^{2}-4 c_{s}^{2}\right)}{\left(c_{p}^{2}-c_{s}^{2}\right)}$

$v=\frac{\left(c_{p}^{2}-2 c_{s}^{2}\right)}{2\left(c_{p}^{2}-c_{s}^{2}\right)}$

Testing was performed on the Calcarenite block with a cross-section of $300 \times 300 \mathrm{~mm}$ and 150 $\mathrm{mm}$ in height labeled as $\mathrm{x}, \mathrm{y}$, and z-axis, respectively, at atmospheric pressure. P- and S-wave measurements were performed at two different locations on each axis. At each of these locations, six readings of $\mathrm{P}$ - and S-wave arrival times were recorded, and the average was used to calculate their respective wave velocities (Table 1 ).

The degree of (velocity) anisotropy was evaluated using a percent difference calculation:

percent difference $=\frac{x_{1}-x_{2}}{\left(\frac{x_{1}+x_{2}}{2}\right)} 100 \%$

The degree of velocity anisotropy from $\mathrm{P}$-wave measurements was $1.4 \%$, while for S-wave velocities the degree of anisotropy was $2.1 \%$. The S-wave velocities with the most discrepancies were the measurements taken through the z-axis. However, the P-wave velocities measured through the z-axis showed a degree of anisotropy of $0.25 \%$. This difference can partially be explained by the difficulty experienced in determining the S-wave arrival time. Nonetheless, a degree of velocity anisotropy of $2.1 \%$ is small and approximately equal to the value of $2.0 \%$ recommended by ASTM D 2845-08 in order to use equations (12) and (13) to calculate the Young's modulus and Poisson's ratio of an isotropic material (Table 1). Pressure dependence of elastic parameters was not considered. 
Table 1. Results from ultrasonic testing.

\begin{tabular}{|c|c|c|c|c|}
\hline Location & Test & $\begin{array}{l}\text { Velocity } \\
{[\mathrm{m} / \mathrm{s}]}\end{array}$ & $\begin{array}{c}\text { Poisson's } \\
\text { Ratio, } v\end{array}$ & $\begin{array}{c}\text { Young's } \\
\text { Modulus, E } \\
\text { [GPa] }\end{array}$ \\
\hline \multirow[b]{2}{*}{$x_{1}$} & $P$ & 2404 & 0.219 & 8.18 \\
\hline & $S$ & 1442 & & \\
\hline Location & Test & $\begin{array}{c}\text { Velocity } \\
{[\mathrm{m} / \mathrm{s}]}\end{array}$ & $\begin{array}{c}\text { Poisson's } \\
\text { Ratio, } v\end{array}$ & $\begin{array}{l}\text { Young's } \\
\text { Modulus, E } \\
\text { [GPa] }\end{array}$ \\
\hline \multirow{2}{*}{$x_{2}$} & $P$ & 2415 & 0.220 & 8.23 \\
\hline & $\mathrm{S}$ & 1446 & & \\
\hline Location & Test & $\begin{array}{c}\text { Velocity } \\
{[\mathrm{m} / \mathrm{s}]}\end{array}$ & $\begin{array}{c}\text { Poisson's } \\
\text { Ratio, } v\end{array}$ & $\begin{array}{l}\text { Young's } \\
\text { Modulus, E } \\
\text { [GPa] }\end{array}$ \\
\hline \multirow[b]{2}{*}{$y_{1}$} & $\mathrm{P}$ & 2414 & 0.223 & 8.20 \\
\hline & $S$ & 1442 & & \\
\hline Location & Test & $\begin{array}{c}\text { Velocity } \\
{[\mathrm{m} / \mathrm{s}]}\end{array}$ & $\begin{array}{c}\text { Poisson's } \\
\text { Ratio, } v\end{array}$ & $\begin{array}{c}\text { Young's } \\
\text { Modulus, E } \\
\text { [GPa] }\end{array}$ \\
\hline \multirow[b]{2}{*}{$y_{2}$} & $P$ & 2429 & 0.219 & 8.34 \\
\hline & $S$ & 1457 & & \\
\hline Location & Test & $\begin{array}{c}\text { Velocity } \\
{[\mathrm{m} / \mathrm{s}]}\end{array}$ & $\begin{array}{c}\text { Poisson's } \\
\text { Ratio, } v\end{array}$ & $\begin{array}{l}\text { Young's } \\
\text { Modulus, E } \\
\text { [GPa] }\end{array}$ \\
\hline \multirow[t]{2}{*}{ 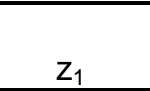 } & $P$ & 2432 & 0.226 & 8.28 \\
\hline & $S$ & 1447 & & \\
\hline Location & Test & $\begin{array}{c}\text { Velocity } \\
{[\mathrm{m} / \mathrm{s}]}\end{array}$ & $\begin{array}{c}\text { Poisson's } \\
\text { Ratio, } v\end{array}$ & $\begin{array}{c}\text { Young's } \\
\text { Modulus, E } \\
\text { [GPa] }\end{array}$ \\
\hline \multirow[b]{2}{*}{$z_{2}$} & $\mathrm{P}$ & 2438 & 0.210 & 8.52 \\
\hline & $S$ & 1477 & & \\
\hline
\end{tabular}

As is shown in Table 1, the elastic parameters $v$ and $E$ range from $0.210-0.226$ and $8.18-8.52$ $\mathrm{GPa}$, respectively, with average values of 0.220 and $8.29 \mathrm{GPa}$.

\subsubsection{Uniaxial compression testing}

Uniaxial compression tests were performed on both right circular cylinders and rectangular prisms. Three cylindrical specimens and one rectangular prism specimen were prepared in accordance with ASTM and ISRM standards and tested within a closed-loop, servo hydraulic 1 MN load frame (MTS Systems, Eden Prairie, MN) at an axial displacement rate of $5 \times 10^{-4} \mathrm{~mm} / \mathrm{s}$. 
The cylindrical specimens were $31.0 \mathrm{~mm}$ in diameter, slightly smaller than the suggested diameter size of $54 \mathrm{~mm}$ but the diameter to grain size ratio was still larger than the recommended value of $10: 1$. The height of the cylindrical specimens ranged from $83.9-89.8 \mathrm{~mm}$, within the desired height to diameter ratio of $2-3$. The rectangular specimen was $35.6 \times 43.0 \mathrm{~mm}$ in crosssection, and $60.0 \mathrm{~mm}$ in height. The ends of the specimens were flat to $0.02 \mathrm{~mm}$ and they did not depart from perpendicularity to the longitudinal axis by more than $\pm 0.05 \mathrm{~mm}$ in $50 \mathrm{~mm}$. The axial force was measured with a load cell (sensitivity $=1 \mathrm{kN} / \mathrm{vdc}$ ) and the uniaxial compressive strength (UCS or $C_{0}$ ) is simply

$U C S=C_{o}=\frac{F_{\max }}{A}$

\section{$\mathrm{A}=$ cross-sectional area $\left[\mathrm{m}^{2}\right]$}

The cylindrical cores were fabricated from each orientation $x, y$ and $z$ of the Calcarenite block. The material was determined to be isotropic in UCS (Table 2).

Table 2. Uniaxial compression results of cylindrical Calcarenite specimens.

\begin{tabular}{|c|c|c|c|}
\hline Orientation & $D[\mathrm{~mm}]$ & $\mathrm{H}[\mathrm{mm}]$ & $\begin{array}{c}\text { UCS } \\
{[\mathrm{MPa}]}\end{array}$ \\
\hline $\mathrm{x}$ & 31.1 & 89.4 & 16.2 \\
\hline $\mathrm{y}$ & 30.9 & 89.8 & 16.2 \\
\hline $\mathrm{z}$ & 30.8 & 83.9 & 16.0 \\
\hline
\end{tabular}

To determine elastic parameters from mechanical loading, a uniaxial compressive test was performed on the rectangular specimen instrumented with axial and transverse strain gages. The specimen was loaded to approximately $50 \%$ of the UCS, within the linear region of material response, and then unloaded. The strains measured during loading were used to calculate the Young's modulus $(E)$ and Poisson's ratio $(v)$ :

$E=\frac{\Delta \sigma_{a}}{\Delta \varepsilon_{a}}$

$v=\frac{-\Delta \varepsilon_{t}}{\Delta \varepsilon_{a}}$

$\Delta \sigma_{\mathrm{a}}=$ increment of axial stress $[\mathrm{MPa}]$

$\Delta \varepsilon_{\mathrm{a}}=$ increment of axial strain $[\mu \varepsilon]$

$\Delta \varepsilon_{\mathrm{t}}=$ increment of transverse strain $[\mu \varepsilon]$ 
The rectangular specimen was fabricated so that axial force was applied along the z-axis of the material. Each of the strain gages were connected to a Wheatstone bridge circuit and were shunt calibrated with a $174,800 \Omega$ resistor; both strain gages had a shunt strain of $\varepsilon_{s}=951.56 \mu \varepsilon$. The specimen was then loaded to approximately $50 \%$ of the UCS. The axial stress - axial strain response is shown in Figure 7.

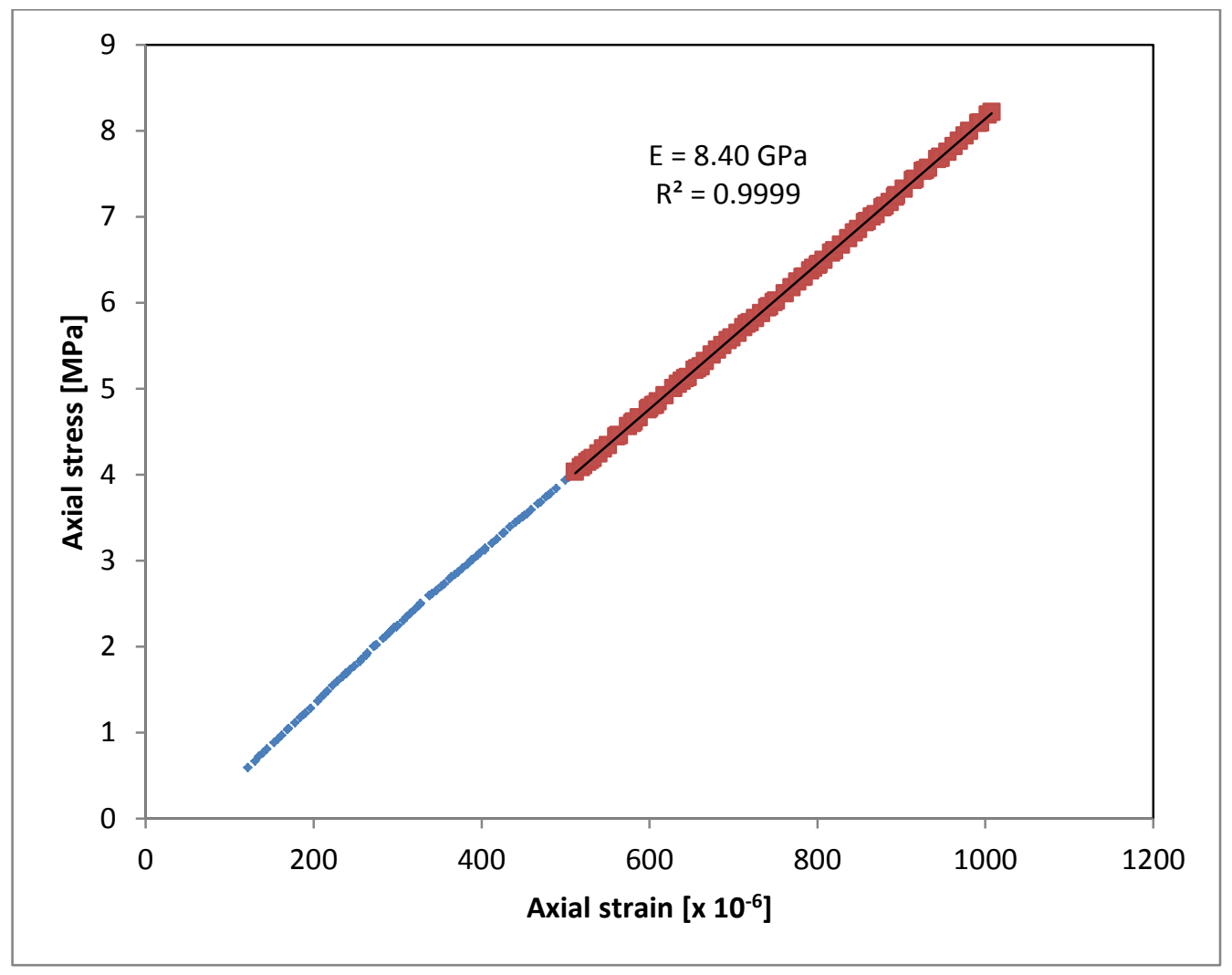

Figure 7. Mechanical response from a uniaxial compression test performed on the rectangular Calcarenite specimen.

The highlighted portion of the axial stress - axial strain curve is the linear portion considered when calculating the Young's modulus (E).

The transverse strain - axial strain curve is shown in Figure 8. The highlighted portion of the curve corresponds to the same region highlighted in the axial stress - axial strain curve. 


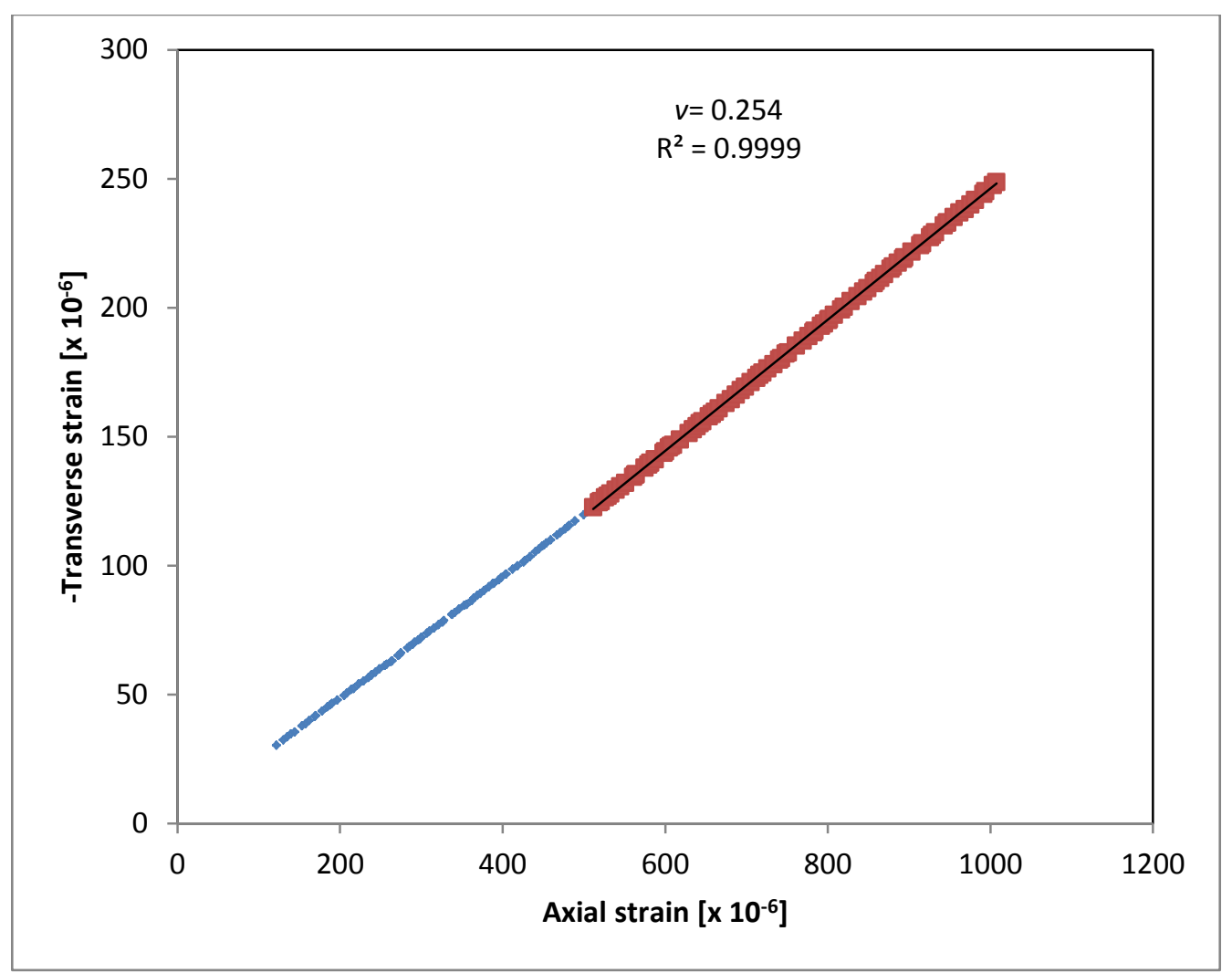

Figure 8. Deformation response from a uniaxial compression test performed on the rectangular Calcarenite specimen.

A comparison of the elastic properties determined by the ultrasonic wave and uniaxial compression testing is presented in Table 3.

Table 3. Comparison of ultrasonic and uniaxial compression testing results.

\begin{tabular}{|l|c|c|}
\hline Testing technique & Young's Modulus [GPa] & Poisson's Ratio \\
\hline Ultrasonic wave & 8.29 & 0.220 \\
\hline Uniaxial compression & 8.40 & 0.254 \\
\hline
\end{tabular}

\subsubsection{Uniaxial tension testing}

According to ASTM D2936-08, direct tensile strength and deformability should be determined from dog-bone shaped specimens of intact rock (Figure 9); having a reduced cross section allows 
fracture to occur away from the ends of the specimen. Another benefit to this design is that the machined flat section in the middle of the specimen is a convenient location for strain gages (1) the numbers in the parenthesis refer to the components labeled in Figure 9, which can be used to measure any bending that may be induced.
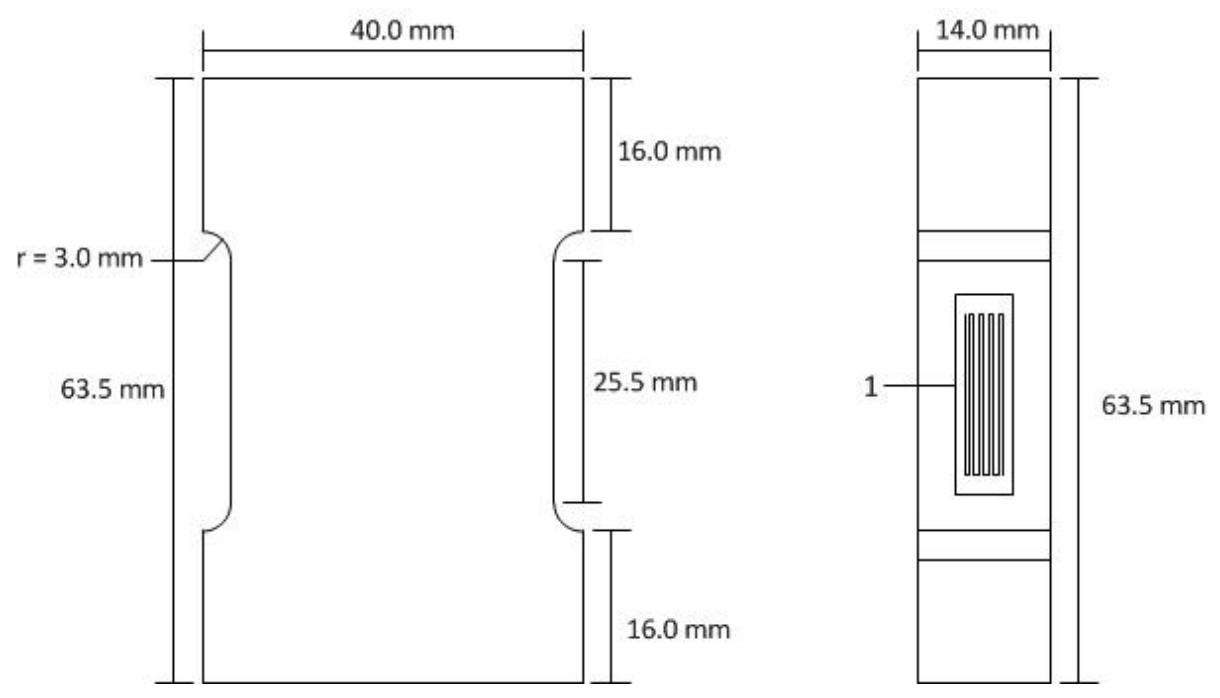

Figure 9. Dimensions of Calcarenite specimen for uniaxial tension testing.

Direct tension testing was performed using the flexible-cable method (Figure 10) suggested by Fairhurst [1961]. The rectangular prism dog-bone shaped specimen was bonded by direct buttjointing, where the specimen end was bonded directly to the surface of the platen using a highstrength epoxy. Another technique for connecting a specimen to the platens is to clamp the specimen to the platen; Hawkes and Mellor [1969] described a method using an aluminum collar that tightens around the specimen end as the cable is connected. The friction grip is generally used on high strength rocks, where simple bonding cannot be used due to limited tensile strength of the epoxy; a drawback to this connection of the platen is that the failure occurs near the connection [Hawkes and Mellor 1969]. Since Calcarenite is a soft rock with an estimated tensile strength of $1-2 \mathrm{MPa}$, it was determined that bonding platens would be the best approach. The test set-up consisted of two steel platens (2) that were bonded to the specimen (1), using the direct butt-jointing technique. The epoxy (6) has a tensile strength of $22.1 \mathrm{MPa}$, more than enough to ensure failure of the specimen before the failure of the epoxy. 
Another important aspect of the flexible cable method is the cable itself. Fairhurst [1961] used a $3 \mathrm{~mm}$ (1/8 in.) wire cable that was unwrapped and soldered directly to a cable grip attached to the steel platen. The technique of "unwrapping" the cable and soldering the now unwrapped strands directly to the cable grip was done to eliminate torsion due to twist of the cable strands. For the experiments, the steel platens were connected to $6 \mathrm{~mm}(0.25 \mathrm{in}$.) diameter steel cables (5) with high-tensile-strength eye-bolts (3) that were threaded directly into the steel platens. The specimen was then fastened into the load frame by attaching the bottom steel cable to the bottom actuator through a high-tensile-strength eye-bolt. To avoid torsion in the specimen, the top cable was attached to a threaded steel ball joint (4), which allowed the specimen to rotate freely $\pm 20^{\circ}$. The ball joint was connected to a $22.25 \mathrm{kN}$ (5000 lb) load cell.

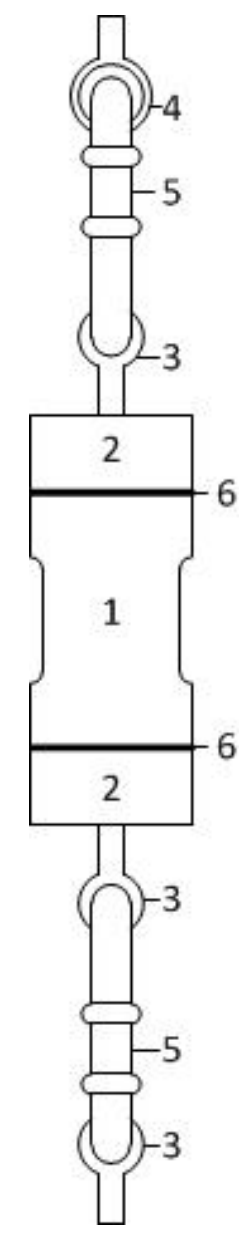

Figure 10. Direct tension testing apparatus. 
A closed-loop, servo controlled test was performed with an average axial displacement of rate of $5 \times 10^{-4} \mathrm{~mm} / \mathrm{s}$. The $1 \mathrm{MN}$ load frame was connected to a data acquisition system that recorded axial force, axial displacement, and output from two axial strain gages located on opposite sides of the specimen in the reduced cross-sectional area. From this test the uniaxial tensile strength, $T$, can be determined using

$$
T=\frac{F_{\max }}{A}
$$

$\mathrm{F}_{\max }=$ maximum tensile load applied $[\mathrm{kN}]$

$\mathrm{A}=$ reduced cross-sectional area $\left[\mathrm{m}^{2}\right]$

One uniaxial tension test was performed on a Calcarenite specimen instrumented with two strain gages. The strain gages on the specimen were connected to a Wheatstone bridge circuit and was shunt calibrated with a $174,800 \Omega$ resistor internal to the signal conditioner; both strain gages had a shunt strain of $\varepsilon_{s}=951.56 \mu \varepsilon$. The direct tensile strength of the material was determined using equation (18)

$$
T=\frac{F_{\max }}{A}=2.06 \mathrm{MPa}
$$

The axial stress was then plotted against the axial strain to determine the Young's modulus in tension of the Calcarenite specimen (Figure 11). 


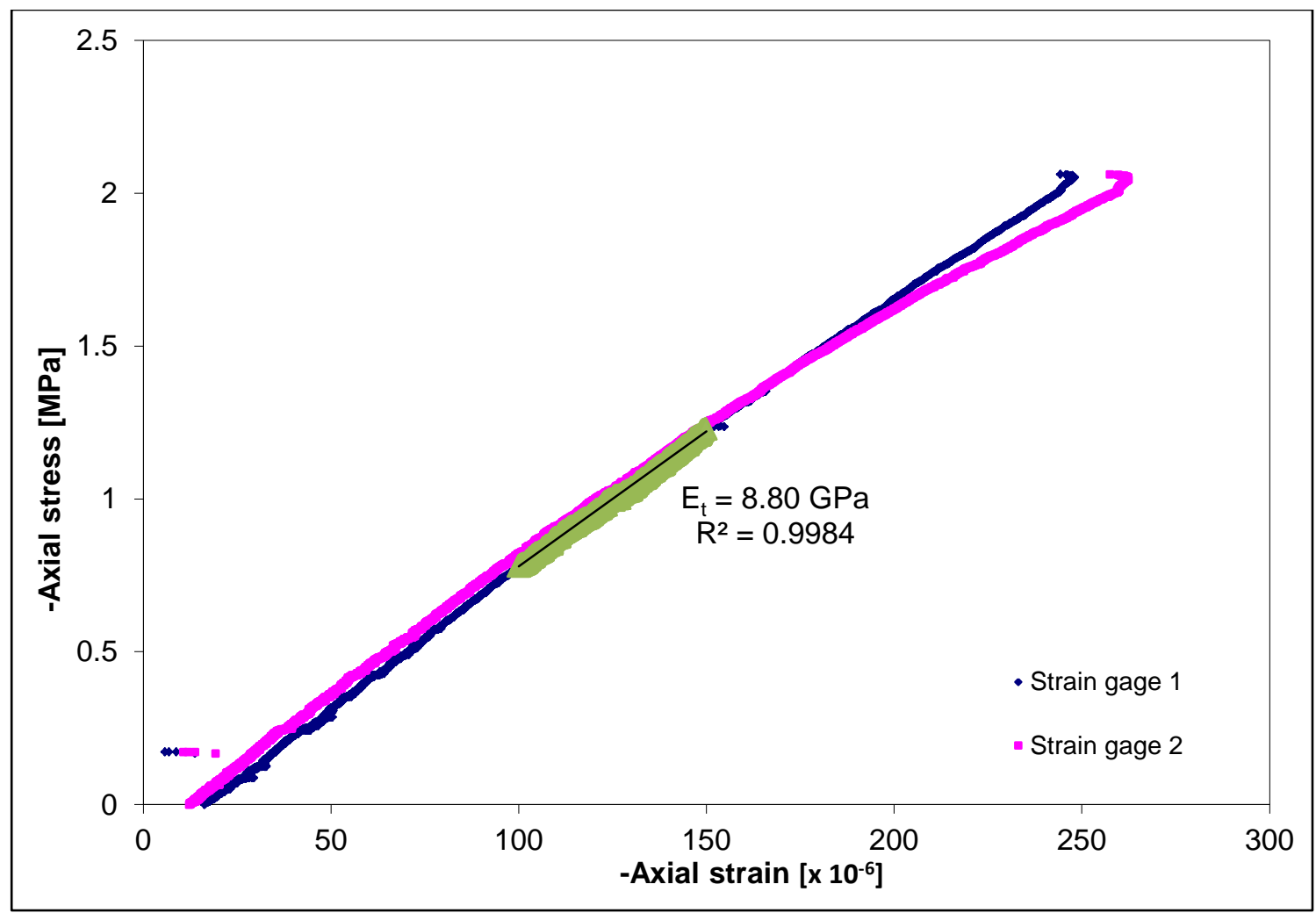

Figure 11. Axial stress - axial strain of two strain gages attached on two sides of the Calcarenite specimen located in the reduced cross-section.

Considering the highlighted portion, axial stress of approximately $0.6-1.4 \mathrm{MPa}$, to be the linear response of the Calcarenite during the direct tension test the Young's modulus in tension was determined to be $8.80 \mathrm{GPa}$. By computing the average percent difference between the two strain gages, it can be seen that the strains on either side of the specimen are within $1.0 \%$ of each other.

Since the strain values are so close to each other throughout the linear section, it can be safely assumed that the test was performed with no bending. As shown in Figure 12, the specimen failed normal to the direction of applied load, which agrees with the known fact that brittle materials fracture on planes normal to the direction of maximum tensile stress (minor principal stress). 

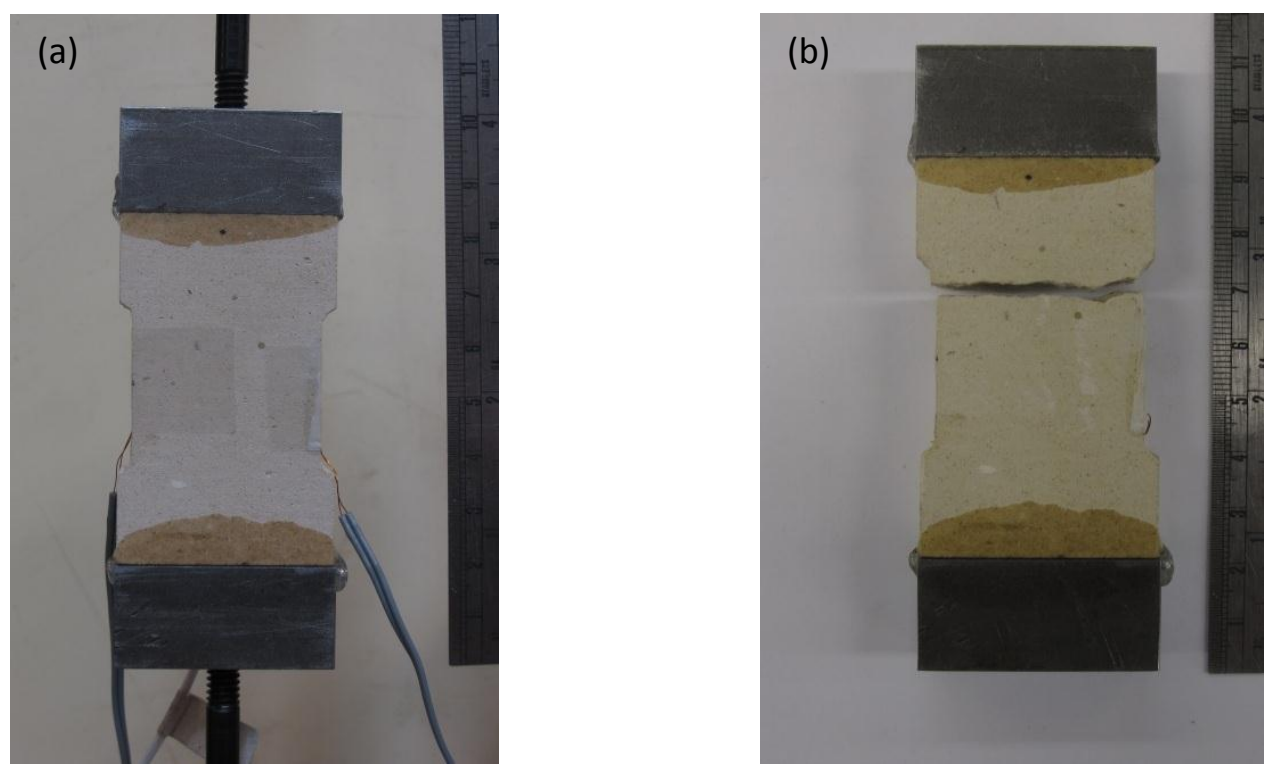

Figure 12. Direct tension specimen (a) before testing; (b) after testing.

\subsection{Shear strength testing}

The shear strength parameters of Calcarenite were determined using two methods: (1) a series of conventional triaxial tests in compression and extension; This allowed for the shear strength parameters to be determined with an evaluation of the intermediate stress effect. (2) Multi-axial tests, where all three principal stresses were different, under a condition of biaxial deformation (plane-strain).

\subsubsection{Conventional triaxial testing}

A Hoek-Franklin cell, Figure 13, is a pressure vessel, often called a triaxial cell, that allows for an application of axial stress independent of radial stress developed through confining pressure. The triaxial cell, manufactured by Engineering Laboratory Equipment International Inc. (ELE International Inc., Loveland, $\mathrm{CO}$ ), was designed for cores $31.0 \mathrm{~mm}$ in diameter and approximately $80 \mathrm{~mm}$ in height. Axial stress was developed by the $1 \mathrm{MN}$ load frame. Radial stress was 
generated by a fluid pressure system, featuring a microcontroller and a screw-type hydraulic intensifier. To increase, decrease, or maintain constant hydraulic pressure, the intensifier piston was advanced or retracted by a stepper motor in response to volume changes elsewhere in the system.

Testing of the specimen (1) involved a specified radial stress induced by hydraulic oil (3). Axial force was applied to the specimen by the load frame through machined platens (4) designed to fit the cell. The surfaces of the specimen in contact with the steel platens were coated with stearic acid lubricant to promote homogeneous deformation (Labuz and Bridell 1993).

An important component of the Hoek-Franklin cell is a one-piece synthetic rubber membrane (2) that is used to isolate the hydraulic oil from the specimen. The membrane protects the specimen from the hydraulic oil and it allows for confining pressure to be applied without leakage. The inside surface of the membrane that comes into contact with the specimen was coated with a layer of stearic acid to reduce friction between the specimen and the membrane, which helped during extrusion of the specimen from the membrane.

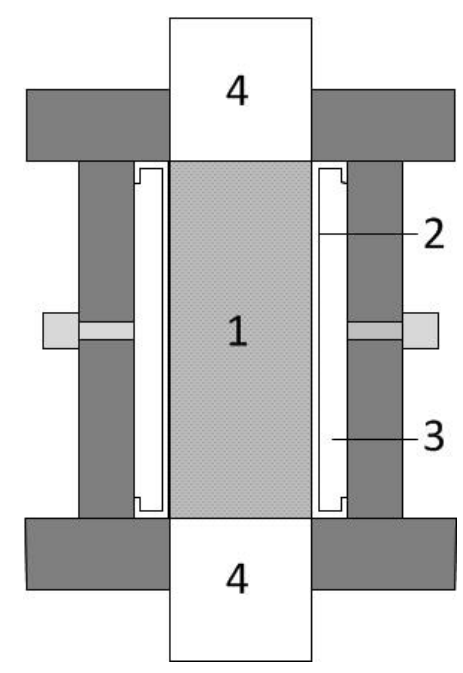

Figure 13. Sketch of Hoek-Franklin triaxial cell.

Closed-loop, computer controlled tests were performed with an average axial displacement rate of $5 \times 10^{-4} \mathrm{~mm} / \mathrm{s}$. The various instrumentation was connected to a data acquisition system that recorded axial force, axial displacement, and in some tests, radial stress at a digitizing frequency of $1 \mathrm{~Hz}$. 
Twenty four Calcarenite specimens were cored from the $300 \times 300 \times 150 \mathrm{~mm}$ block. Because the rock is isotropic, within $2 \%$ in elastic properties, the orientation of the cores with respect to the block geometry was selected to optimize the number of specimens. Thus, the specimens were cored through the $150 \mathrm{~mm}$ length, z-axis, using a diamond tipped $31 \mathrm{~mm}$ core barrel that was cooled using water. The cores were then cut using a masonry saw equipped with a diamond blade to an approximate length of $82 \mathrm{~mm}$. Once the cores were cut to length, they were prepared in accordance to ISRM standards: the ends were ground flat to within $0.02 \mathrm{~mm}$ and they did not depart from perpendicularity to the longitudinal axis of the specimen by more than $\pm 0.05 \mathrm{~mm}$ in $50 \mathrm{~mm}$. The specimen dimensions were $31.0 \mathrm{~mm}$ in diameter and the height ranged from 80.21 $-82.34 \mathrm{~mm}$.

\subsubsection{Plane-strain testing}

The UMN plane-strain compression apparatus, Figure 14, allows for testing of a specimen in a multi-axial setting, where all three principal stresses are different [Labuz et al. 1997]. The device also features a low friction linear bearing called a sled (2) that is useful for evaluating post-failure response. The axial force applied to the specimen (1) is measured by a load cell (4) located inside the pressure chamber. The axial displacement, $\Delta \mathrm{u}_{\mathrm{y}} \neq 0$, and lateral displacement, $\Delta \mathrm{u}_{\mathrm{x}} \neq 0$, are measured by LVDTs (5). The plane-strain deformation, $\Delta \mathrm{u}_{\mathrm{z}} \approx 0$, is enforced by a thick-walled cylinder called a biaxial frame (3). The specimen is secured within the biaxial frame by a set of wedges. The biaxial frame is instrumented with strain gages, which allows for a measure of the intermediate principal stress acting on the specimen.

The specific stiffness of the biaxial frame is determined by calibrating the frame using materials with known properties (aluminum, plexiglass):

$\varepsilon_{z}=\frac{\varepsilon_{\theta}^{f}}{k^{f}}$

where

$\varepsilon_{z}=$ the intermediate principal strain of the specimen

$\varepsilon_{\theta}{ }^{f}=$ the strain measured on the frame

$k^{f}=$ specific stiffness of the biaxial frame $\left(k^{f}=0.180\right.$ or 0.174 depending on specimen size $)$ 
By knowing the intermediate principal strain acting on the specimen, the intermediate principal stress, $\sigma_{\mathrm{z}}$, can be calculated using generalized Hooke's law:

$\sigma_{z}=\varepsilon_{z} E+v\left(\sigma_{x}+\sigma_{y}\right)$

where $\sigma_{\mathrm{y}}=$ axial stress $[\mathrm{MPa}]$ and $\sigma_{\mathrm{x}}=$ confining stress $[\mathrm{MPa}]$.

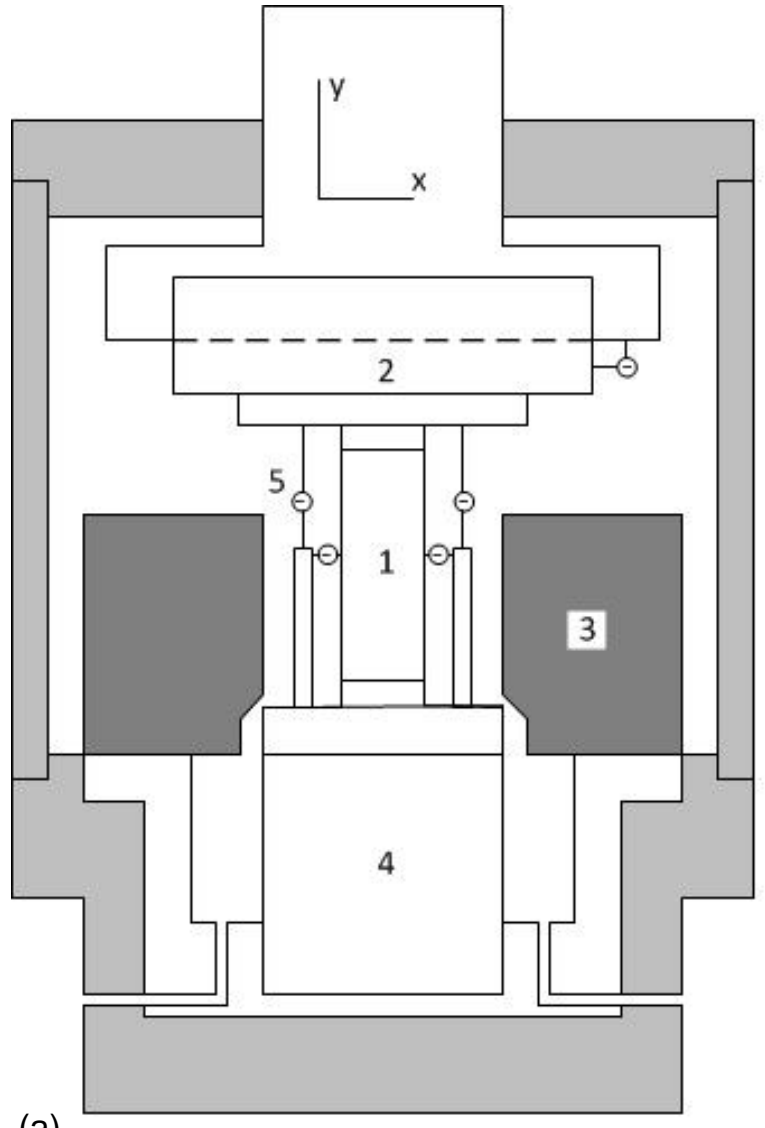

(a)

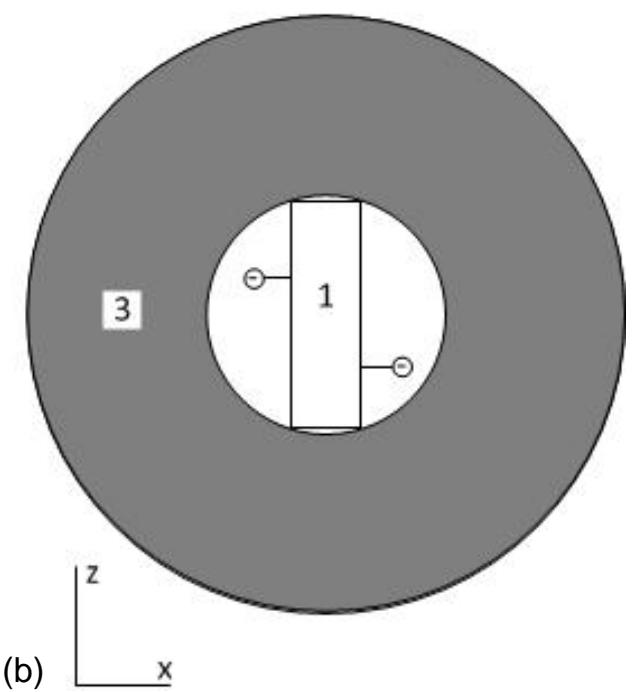

Figure 14. Sketch of UMN Plane-Strain Apparatus. (a) Elevation view. (b) Plan view [Labuz et al. 1997].

Two Calcarenite specimens were cut from the same $300 \times 300 \times 150 \mathrm{~mm}$ block from which the triaxial specimens were cored. Similar to the triaxial specimens, the Calcarenite specimens were prepared such that the axial load was applied parallel to the z-axis. The first specimen was prepared with a cross-section of $100.47 \times 27.98 \mathrm{~mm}$ and $74.93 \mathrm{~mm}$ in height, while the second specimen had a cross-section of $100.69 \times 41.91 \mathrm{~mm}$ and $99.88 \mathrm{~mm}$ in height. Each specimen was prepared to the same standards as the triaxial specimen. All of the faces of the specimen were ground such that opposite sides were parallel and adjacent sides were perpendicular within 
$\pm 0.01 \mathrm{~mm}$ in $100 \mathrm{~mm}$. Two brass tabs, one $4.8 \mathrm{~mm}$ in diameter and $1.6 \mathrm{~mm}$ thick and one $13 \mathrm{~mm}$ in diameter and $1.6 \mathrm{~mm}$ thick were glued to the $100.47 \times 74.93 \mathrm{~mm}$ and $100.69 \times 99.88 \mathrm{~mm}$ faces. The large brass tabs provided firm contact locations for the lateral LVDTs while the small brass taps provided a location to attach an acoustic emission [AE] sensor, which was used to monitor microseismic activity (Figure 15). However, there were very few AE signals recorded during the experiments and the results will not be discussed.

The specimens were assembled with the upper and lower steel platens contacting the $100.47 \mathrm{x}$ $27.98 \mathrm{~mm}$ or $100.69 \times 41.91 \mathrm{~mm}$ faces, depending on the specimen being prepared. Two steel plates, $3 \mathrm{~mm}$ thick, were placed against the $27.98 \times 74.93 \mathrm{~mm}$ or $44.61 \times 99.88 \mathrm{~mm}$ faces. The four surfaces in contact with steel platens were coated with stearic acid to reduce friction (Labuz and Bridell 1993). The specimen and platens were held together with a custom jig and a thin layer of polyurethane was applied to one side of the specimen on the $100.47 \times 74.93 \mathrm{~mm}$ or $100.69 \times 99.88 \mathrm{~mm}$ face. The specimen was then placed in an oven at $50^{\circ} C$ for 12 hours to promote curing of the polyurethane. The specimen was then removed from the oven and a thin layer of polyurethane was applied to the other $100.47 \times 74.93 \mathrm{~mm}$ or $100.69 \times 99.88 \mathrm{~mm}$ face of the specimen while the specimen was still warm. The specimen was then placed in the oven for another 12 hours (Figure 15).

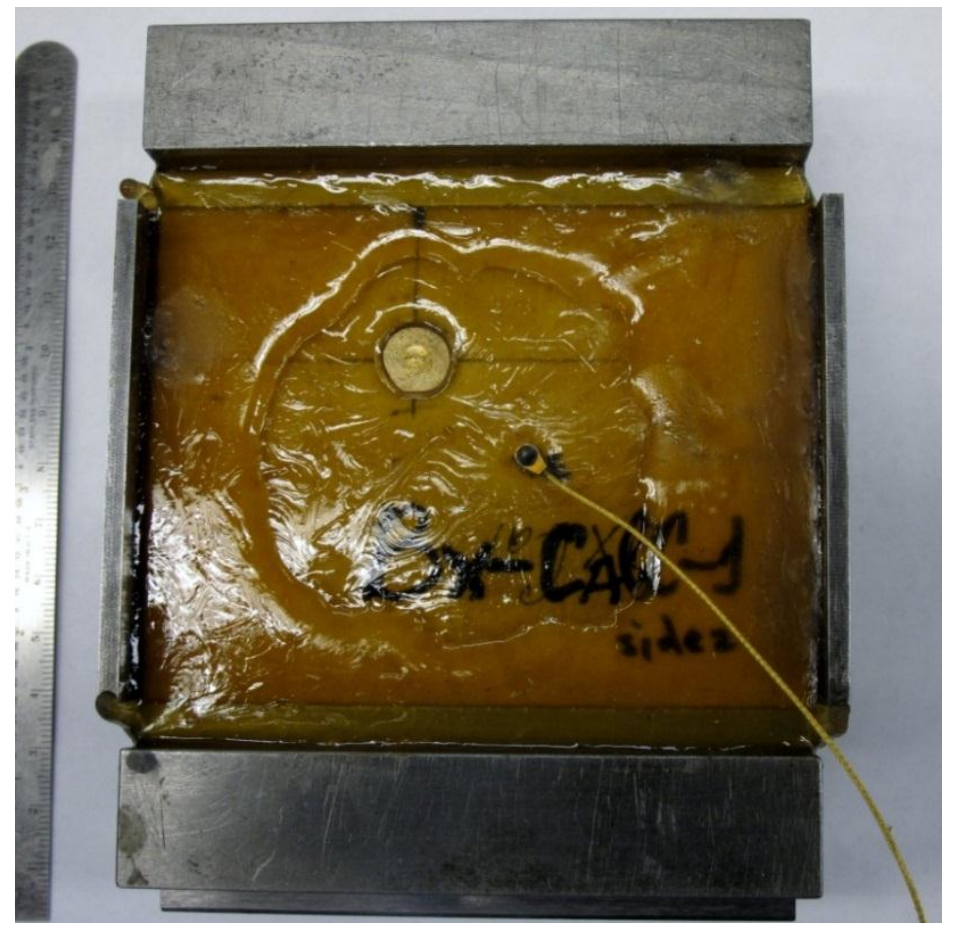

Figure 15. Biaxial test specimen with large brass tab exposed and AE sensor attached. 


\subsection{Strength Parameters}

\subsection{Stress invariants $p$ and $q$}

The failure criterion of a material can be written as a mathematical function of the components of the stress state, $\sigma_{\mathrm{ij}}$ :

$F\left(\sigma_{x}, \sigma_{y}, \sigma_{z}, \sigma_{x y}, \sigma_{x z}, \sigma_{y z}\right)=0$

For an isotropic material, the state of stress at failure does not depend on the axes selected, since by the definition of isotropy, material response is the same in all directions. Thus, the failure criterion for an isotropic material is invariant, and it can be expressed as a function of principal stresses, $\sigma_{\mid}, \sigma_{\|}, \sigma_{\| I}$ :

$F\left(\sigma_{1}, \sigma_{2}, \sigma_{3}\right)=0$

Note that a failure criterion can be dependent on one, two, or all three principal stresses. For example, the $\mathrm{MC}$ failure criterion is dependent on only the major and minor principal stresses.

It is often convenient to express a failure criterion in terms of the stress invariants, $p$ and $q$ :

$p=\frac{1}{3}\left(I_{1}\right)=\frac{1}{3}\left(\sigma_{1}+\sigma_{2}+\sigma_{3}\right)$

where $I_{1}$ is the first invariant of the stress tensor $\sigma_{\mathrm{ij}}$, and

$q=\sqrt{3 J_{2}}=\sqrt{\frac{1}{2}\left[\left(\sigma_{1}-\sigma_{2}\right)^{2}+\left(\sigma_{2}-\sigma_{3}\right)^{2}+\left(\sigma_{3}-\sigma_{1}\right)^{2}\right]}$

where $J_{2}$ is the second invariant of the stress deviator $S_{i j}=\sigma_{i j}-p \delta_{i j}$ and $\delta_{i j}=$ kronecker delta.

Rock is typically sampled as a core - a right circular cylinder - and a common stress state in testing the material is associated with two principal stresses being equal. This is known as conventional triaxial testing, where an axial stress $\sigma_{a}$ is applied along the longitudinal axis of the cylindrical specimen, and a radial stress $\sigma_{r}$ is applied by confining pressure. The state of stress for conventional triaxial experiments in compression, when the specimen experiences axial shortening, is $\sigma_{l}=\sigma_{\mathrm{a}}, \sigma_{\|}=\sigma_{\| I}=\sigma_{\mathrm{r}}$. For triaxial extension experiments, the specimen experiences axial elongation, and the state of stress is $\sigma_{I}=\sigma_{\|}=\sigma_{\mathrm{r}}, \sigma_{\| I}=\sigma_{\mathrm{a}}$. The stress invariants, $p$ and $q$, for conventional triaxial testing are simply 
$p=\frac{1}{3}\left(\sigma_{a}+\sigma_{r}+\sigma_{r}\right)=\frac{\sigma_{a}+2 \sigma_{r}}{3}$

$q=\sqrt{\frac{1}{2}\left[\left(\sigma_{a}-\sigma_{r}\right)^{2}+\left(\sigma_{r}-\sigma_{r}\right)^{2}+\left(\sigma_{r}-\sigma_{a}\right)^{2}\right]}=\sqrt{\frac{1}{2}\left[2 \sigma_{a}^{2}+2 \sigma_{r}^{2}-4 \sigma_{a} \sigma_{r}\right]}=\sigma_{a}-\sigma_{r}$

The value of $q$ will be either positive or negative depending on the type of triaxial experiment performed. In triaxial compression, $\sigma_{\mathrm{a}}>\sigma_{\mathrm{r}}$, and $q>0$ and in triaxial extension, $\sigma_{\mathrm{a}}<\sigma_{\mathrm{r}}$, and $q<0$.

In a $p-q$ diagram (Figure 16), a linear failure criterion can be expressed by

$q=m_{c, e} p+b_{c, e}$

where the subscripts are included to represent failure in either triaxial compression or extension.

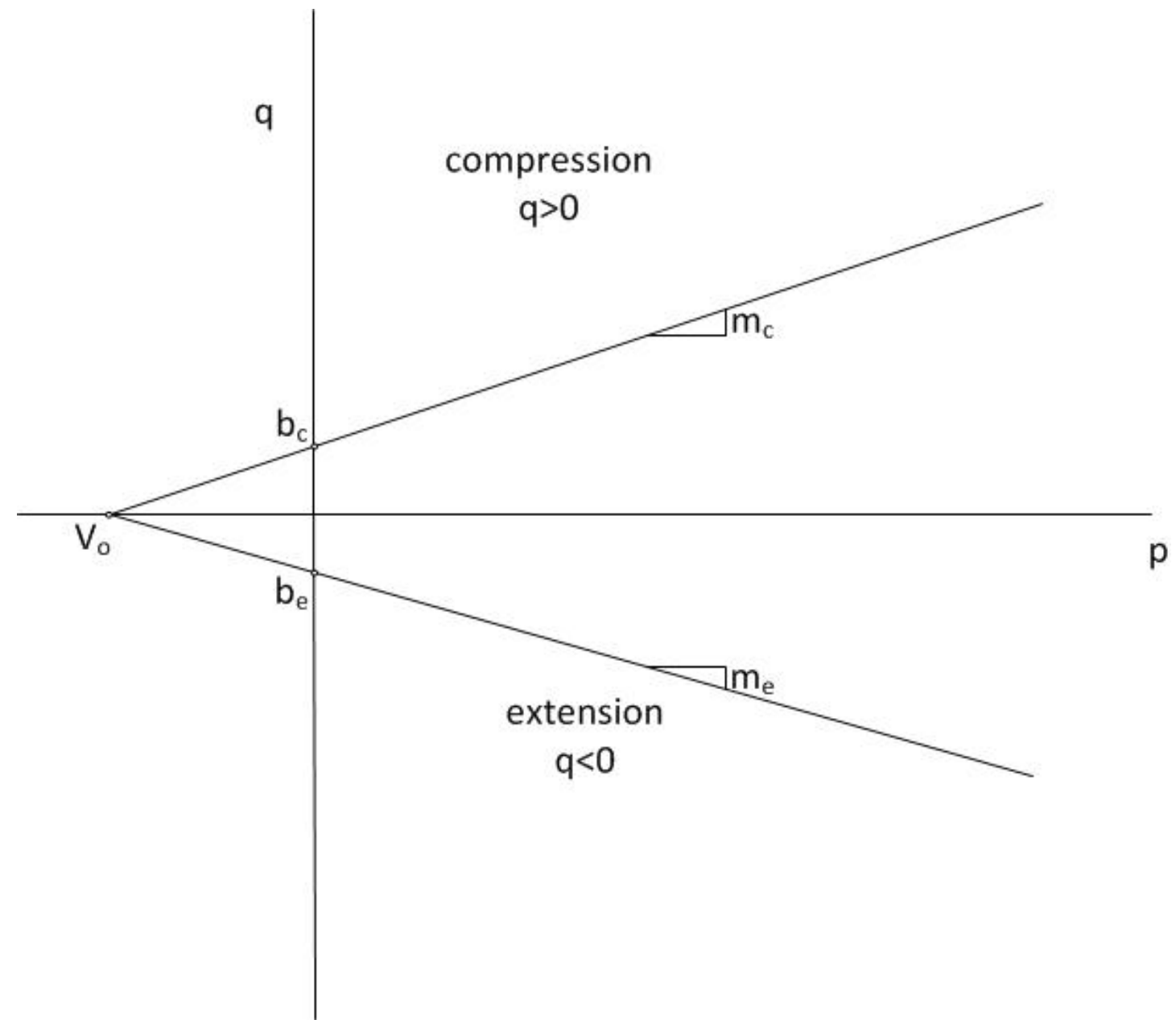

Figure 16. Failure surface described by compression and extension results. 
The MC strength parameters $\phi$ and $c$ can be related to $\mathrm{m}_{\mathrm{c}, \mathrm{e}}$ and $\mathrm{b}_{\mathrm{c}, \mathrm{e}}$. For example, considering the compression line, $\sigma_{I}=\sigma_{a}$ and $\sigma_{I I I}=\sigma_{r}$; equation (6) can be rewritten as

$\left(\sigma_{a}-\sigma_{r}\right)=\left(\sigma_{a}+\sigma_{r}\right) \sin \phi_{c}+2 c_{c} \cos \phi_{c}$

Expanding and rearranging, equation (25) becomes

$\left(\sigma_{a}-\sigma_{r}\right)\left(3-\sin \phi_{c}\right)=2\left(\sigma_{a}+2 \sigma_{r}\right) \sin \phi_{c}+6 c_{c} \cos \phi_{c}$

which can be written in terms of $p$ and $q$

$q=\frac{6 \sin \phi_{c}}{3-\sin \phi_{c}} p+\frac{6 c_{c} \cos \phi_{c}}{3-\sin \phi_{c}}$

The extension line can be solved in a similar manner:

$q=-\frac{6 \sin \phi_{e}}{3+\sin \phi_{e}} p-\frac{6 c_{e} \cos \phi_{e}}{3+\sin \phi_{e}}$

The strength parameters, $\phi_{c}$ and $\phi_{e}$, determined from conventional triaxial compression and extension experiments may not be equal.

\subsection{Triaxial testing results}

Twenty-four conventional triaxial tests were performed on Calcarenite specimens fabricated from a block sample of Calcarenite. Of the twenty-four tests, twelve were performed in compression, $\sigma_{\text {I }}$ $=\sigma_{\mathrm{a}}$ and $\sigma_{\|}=\sigma_{\| I}=\sigma_{\mathrm{r}}$. Six of these compression tests were performed as compression loading tests, where $\sigma_{a}$ was increased $\left(\Delta \sigma_{a}>0\right)$ from $\sigma_{a}=\sigma_{r}$ until failure and $\sigma_{r}=$ constant; six were performed in compression unloading, $\sigma_{r}$ is decreased $\left(\Delta \sigma_{r}<0\right)$ until failure with $\sigma_{a}=$ constant. The remaining twelve tests were performed in extension, $\sigma_{I}=\sigma_{2}=\sigma_{\mathrm{r}}$ and $\sigma_{\| I}=\sigma_{\mathrm{a}}$. Six of these were performed in extension loading, $\sigma_{r}$ is increased $\left(\Delta \sigma_{r}>0\right)$ until failure and $\sigma_{a}=$ constant; six were conducted in extension unloading, where $\sigma_{a}$ was decreased $\left(\Delta \sigma_{a}<0\right)$ until failure and $\sigma_{r}=$ constant. Compression loading and extension unloading were performed at an axial displacement rate of $5 \times 10^{-4} \mathrm{~mm} / \mathrm{s}$. The results from the compression and extension experiments are presented in Table 4 and Table 5, respectively. 
Table 4. Results from triaxial compression experiments $\left(\sigma_{I}=\sigma_{\mathrm{a}}, \sigma_{\|}=\sigma_{\| I}=\sigma_{\mathrm{r}}\right)$.

\begin{tabular}{|c|c|c|c|c|c|}
\hline Test & $\begin{array}{c}\text { Stress } \\
\text { path }\end{array}$ & $\begin{array}{c}\text { Radial stress } \\
{[\mathrm{MPa}]}\end{array}$ & $\begin{array}{c}\text { Axial stress } \\
{[\mathrm{MPa}]}\end{array}$ & $\begin{array}{c}p \\
{[\mathrm{MPa}]}\end{array}$ & $\begin{array}{c}q \\
{[\mathrm{MPa}]}\end{array}$ \\
\hline $1^{*}$ & $\mathrm{~L}$ & 5.0 & 23.9 & 11.3 & 18.9 \\
\hline $3^{*}$ & $\mathrm{~L}$ & 2.5 & 19.3 & 8.1 & 16.8 \\
\hline 4 & $\mathrm{~L}$ & 1.25 & 17.4 & 6.6 & 16.1 \\
\hline 8 & $\mathrm{~L}$ & 1.25 & 19.0 & 7.2 & 17.8 \\
\hline 9 & $\mathrm{~L}$ & 2.5 & 22.3 & 9.1 & 19.8 \\
\hline 10 & $\mathrm{~L}$ & 5.0 & 25.5 & 11.8 & 20.5 \\
\hline $14^{*}$ & $\mathrm{U}$ & 6.2 & 24.5 & 12.3 & 18.3 \\
\hline 16 & $\mathrm{U}$ & 2.1 & 20.5 & 8.2 & 18.5 \\
\hline 17 & $\mathrm{U}$ & 0.9 & 18.0 & 6.6 & 17.2 \\
\hline 18 & $\mathrm{U}$ & 3.90 & 24.5 & 10.8 & 20.6 \\
\hline $19^{*}$ & $\mathrm{U}$ & 1.10 & 20.5 & 7.5 & 19.4 \\
\hline 20 & $\mathrm{U}$ & 0.7 & 18.0 & 6.5 & 17.3 \\
\hline
\end{tabular}

Not all values presented were used in calculation of material properties, as tests marked with * indicate failure occurring along a weak plane or no failure plane was observed. Figure 17.a shows an example of a specimen influenced by a weak plane, while Figure 17.b shows a through-going failure plane.
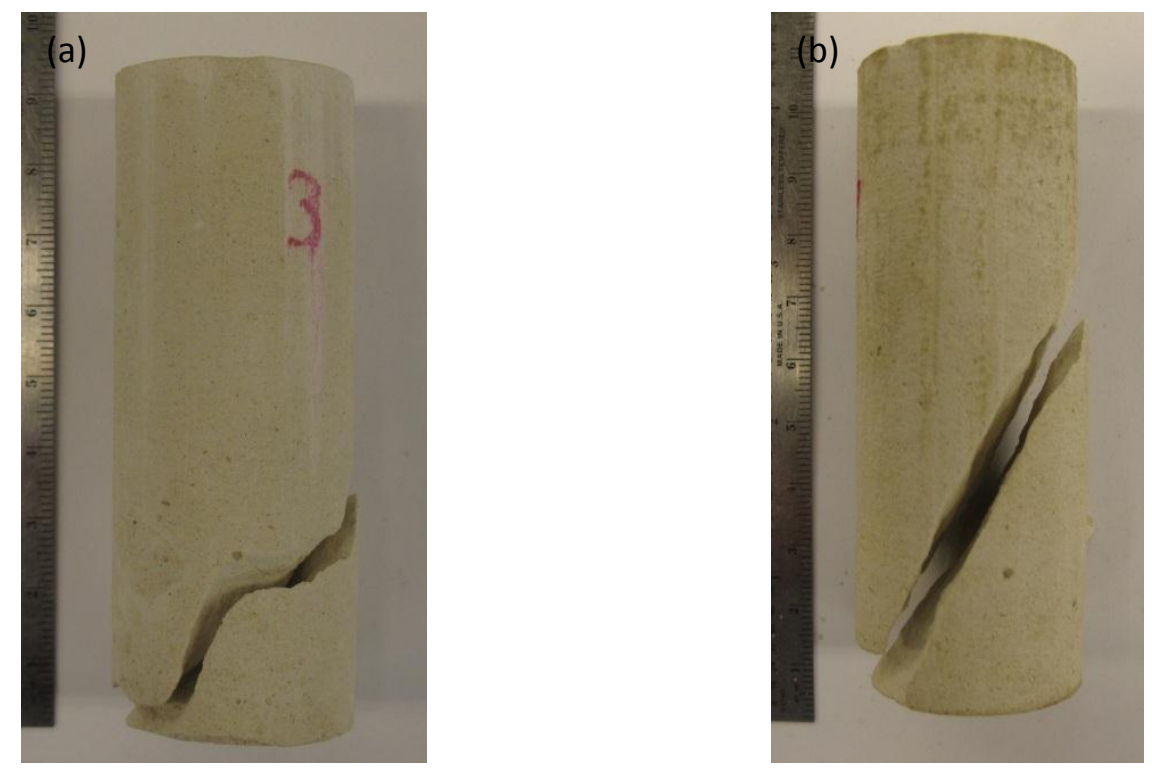

Figure 17. (a) Specimen influenced by a weak plane; (b) typical compression failure plane. 
Mohr-Coulomb theory predicts the angle of the failure plane to be $\beta=45+\frac{\phi}{2}$ from the minor principal stress.

Table 5. Results from triaxial extension experiments $\left(\sigma_{l}=\sigma_{\|}=\sigma_{\mathrm{r}}\right.$ and $\left.\sigma_{\| I}=\sigma_{\mathrm{a}}\right)$.

\begin{tabular}{|c|c|c|c|c|c|}
\hline Test & $\begin{array}{c}\text { Stress } \\
\text { path }\end{array}$ & $\begin{array}{c}\text { Radial stress } \\
{[\mathrm{MPa}]}\end{array}$ & $\begin{array}{c}\text { Axial stress } \\
{[\mathrm{MPa}]}\end{array}$ & $\begin{array}{c}p \\
{[\mathrm{MPa}]}\end{array}$ & $\begin{array}{c}q \\
{[\mathrm{MPa}]}\end{array}$ \\
\hline 15 & $\mathrm{~L}$ & 19.5 & 1.2 & 13.4 & -18.3 \\
\hline 21 & $\mathrm{~L}$ & 18.0 & 0.3 & 12.1 & -17.8 \\
\hline 22 & $\mathrm{~L}$ & 23.7 & 2.6 & 16.7 & -21.1 \\
\hline 23 & $\mathrm{~L}$ & 24.0 & 2.6 & 17.1 & -20.6 \\
\hline 24 & $\mathrm{~L}$ & 20.0 & 1.2 & 13.7 & -18.8 \\
\hline 25 & $\mathrm{~L}$ & 16.0 & 0.1 & 16.9 & -21.4 \\
\hline 5 & $\mathrm{U}$ & 16.8 & 0.1 & 11.2 & -16.6 \\
\hline 6 & $\mathrm{U}$ & 18.0 & 1.1 & 12.4 & -16.9 \\
\hline 7 & $\mathrm{U}$ & 21.8 & 2.7 & 15.4 & -19.1 \\
\hline $11^{*}$ & $\mathrm{U}$ & 16.8 & 1.4 & 11.6 & -15.4 \\
\hline 12 & $\mathrm{U}$ & 18.0 & 0.4 & 12.1 & -17.6 \\
\hline 13 & $\mathrm{U}$ & 21.8 & 2.5 & 15.3 & -19.2 \\
\hline
\end{tabular}
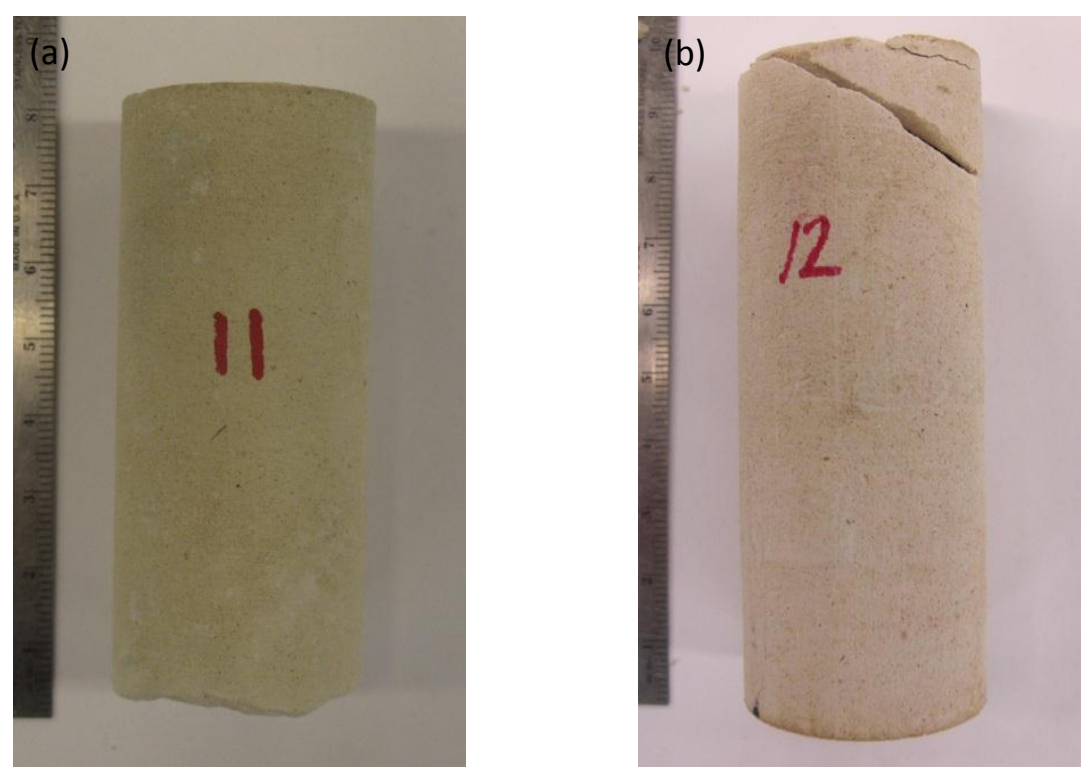

Figure 18 (a) Specimen influenced by a weak plane; (b) typical extension failure plane. 
The photographs presented in Figure 18 demonstrate extension failure planes that are either influenced by weak planes (a) or that develop without influence of weak planes (b). The orientation of the failure plane is governed by the equation $=45+\frac{\phi}{2}$, but it is important to note in extension tests the orientation of the minor principal stress is parallel to the vertical axis of the specimen. The data obtained from conventional triaxial experiments is presented in (Figure 19).

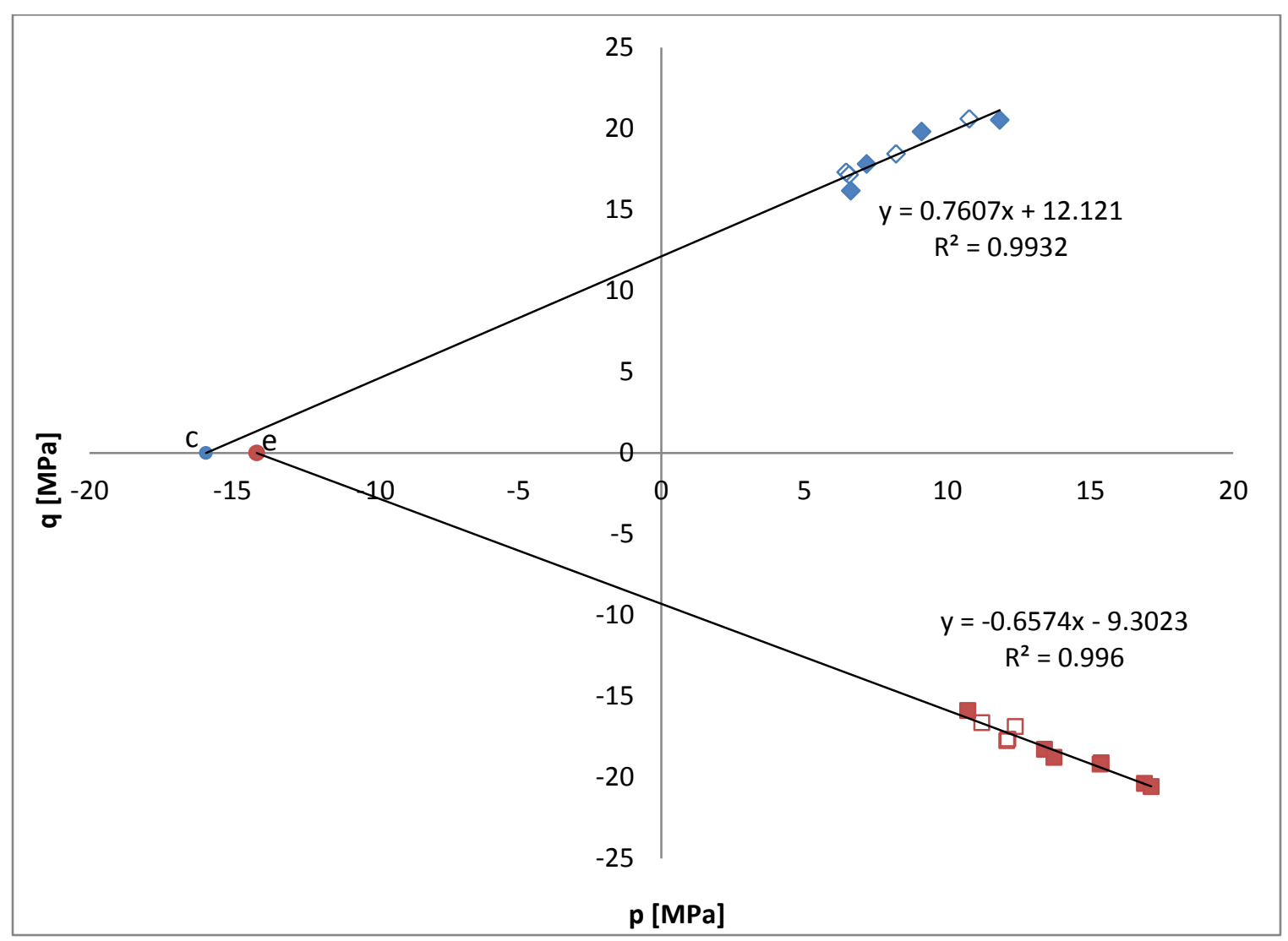

Figure 19. Triaxial compression and extension results presented in $p$ - $q$ form. Open data points represent tests run in unloading stress path.

From the lines of least-squares in Figure 19, the angle of internal friction and cohesion for compression can be determined using equation (30):

$$
\begin{gathered}
m_{c}=\frac{6 \sin \left(\phi_{c}\right)}{3-\sin \left(\phi_{c}\right)}=0.7607 \\
\phi_{c}=\sin ^{-1}\left(\frac{3 m_{c}}{\left(6+m_{c}\right)}\right)=19.7^{\circ}
\end{gathered}
$$




$$
\begin{gathered}
b_{c}=\frac{6 c_{c} \cos \left(\phi_{c}\right)}{3-\sin \left(\phi_{c}\right)}=12.12 \mathrm{MPa} \\
c_{c}=\frac{b_{c}\left(3-\sin \left(\phi_{c}\right)\right)}{6 \cos \left(\phi_{c}\right)}=5.71 \mathrm{MPa}
\end{gathered}
$$

Similarly, $\phi_{\mathrm{e}}$ and $c_{c}$ were calculated using equation (31): $\phi_{\mathrm{e}}=21.7^{\circ}$ and $c_{e}=5.62 \mathrm{MPa}$. Thus, it appears that this rock exhibits a (small) effect due to the intermediate stress.

\subsection{Equal triaxial tension state}

A difficulty in interpreting the data comes from the unequal intercepts on the $p$-axis (Figure 19). When constructing a failure surface, all planes must intersect at one point referred to as the vertex or the stress state of equal triaxial tension. Although this stress state is difficult to generate in an experiment, it must be defined to describe the failure surface.

The method of defining the least squares fit was modified by adding the constraint that the lines must intersect the $p$-axis at the same point $\left(V_{o}\right)$ and $V_{o}$ must be within the original $p$-axis intersections for the compression and extension lines, $c$ and e respectively (Figure 20), new equations of the lines of best fit can be calculated. 


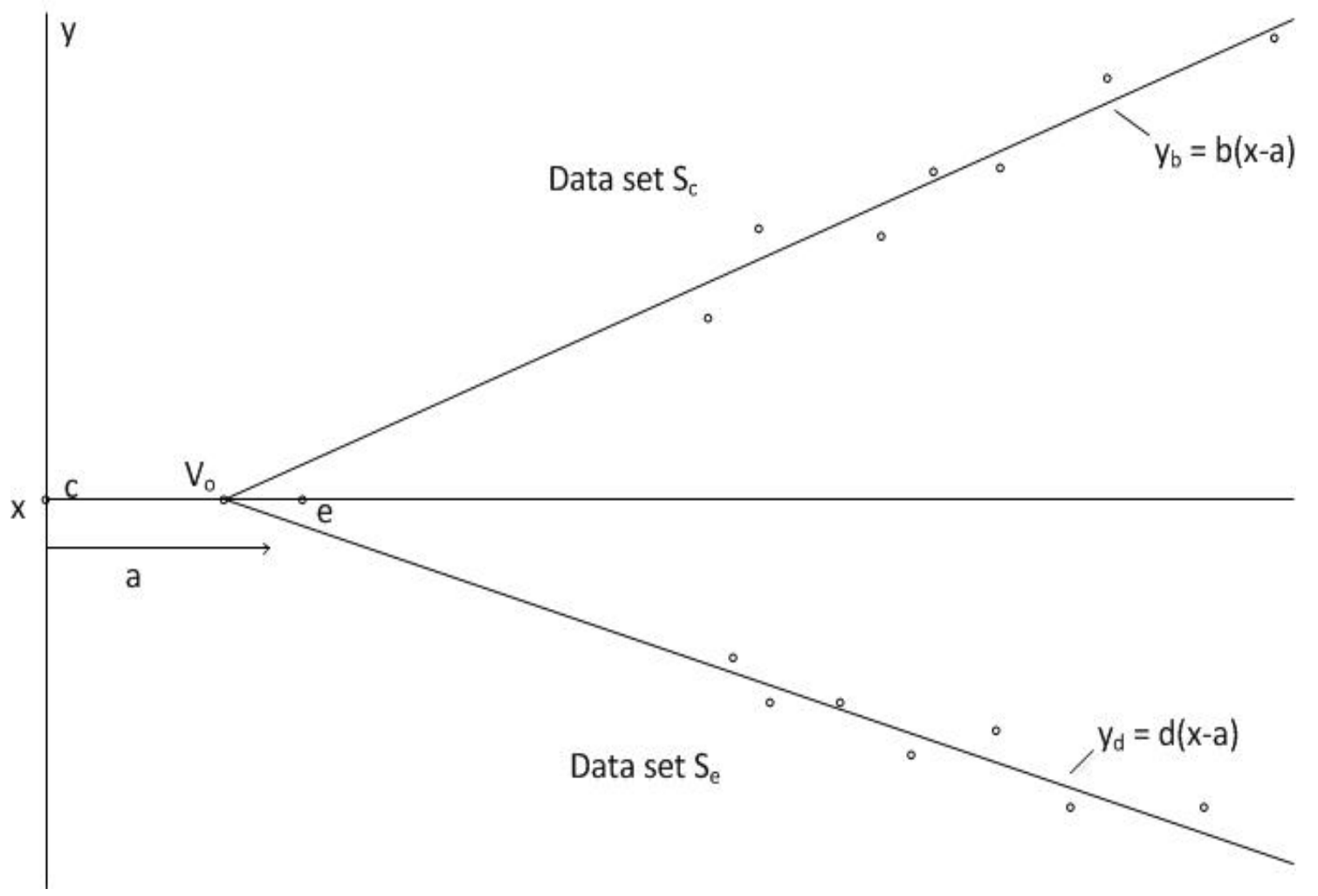

Figure 20. Sketch of least squares lines intersecting the $x$-axis at $V_{o}$.

The point $V_{o}$ and corresponding lines of best fit to the data sets, $S_{c}$ (compression) and $S_{e}$ (extension), can be determined by minimizing

$f(a, b, d)=\sum_{S_{c}}\left(y_{S_{c} \text { data }}\left(x_{i}\right)-y_{b}\left(x_{i}\right)\right)^{2}+\sum_{S_{e}}\left(y_{S_{e} \text { data }}\left(x_{i}\right)-y_{d}\left(x_{i}\right)\right)^{2}$

where $a$ is used to calculate the location of the vertex $V_{o}$ and $b$ and $d$ are the slopes of the lines of best fit for the compression and extension data, respectively.

Another method used to determine the vertex and the lines of best fit was to maximize the value of the square of the correlation coefficient $\left(R^{2}\right)$ for each line. For this problem, there is one additional constraint. The values of $R^{2}$ for each line must be as close to each other as possible, this is done so that one line does not fit perfectly while one does not match the data well. By again examining Figure 20, equation (33) was determined. By maximizing equation (33), the optimal vertex point and the corresponding lines of best fit can be calculated. 
$f(a, b, c)=1-\frac{\sum_{S_{c}}\left(y_{S_{c} \text { data }}\left(x_{i}\right)-y_{b}\left(x_{i}\right)\right)^{2}}{\sum_{S_{c}}\left(y_{S_{c} \text { data }}\left(x_{i}\right)-\operatorname{mean}\left(y_{S_{c} \text { data }}\right)\right)^{2}}+1-\frac{\sum_{S_{e}}\left(y_{S_{e} \text { data }}\left(x_{i}\right)-y_{d}\left(x_{i}\right)\right)^{2}}{\sum_{S_{e}}\left(y_{S_{e} \text { data }}\left(x_{i}\right)-\text { mean }\left(y_{S_{e} \text { data }}\right)\right)^{2}}$

From both methods, it was determined that the lines of best fit for the data obtained during testing intersected the $p$-axis $\left(V_{o}\right)$ at $-15.16 \mathrm{MPa}$. The line corresponding to the compression data set had a slope of 0.785 , while the line corresponding to the extension data set had a slope of -0.635 .

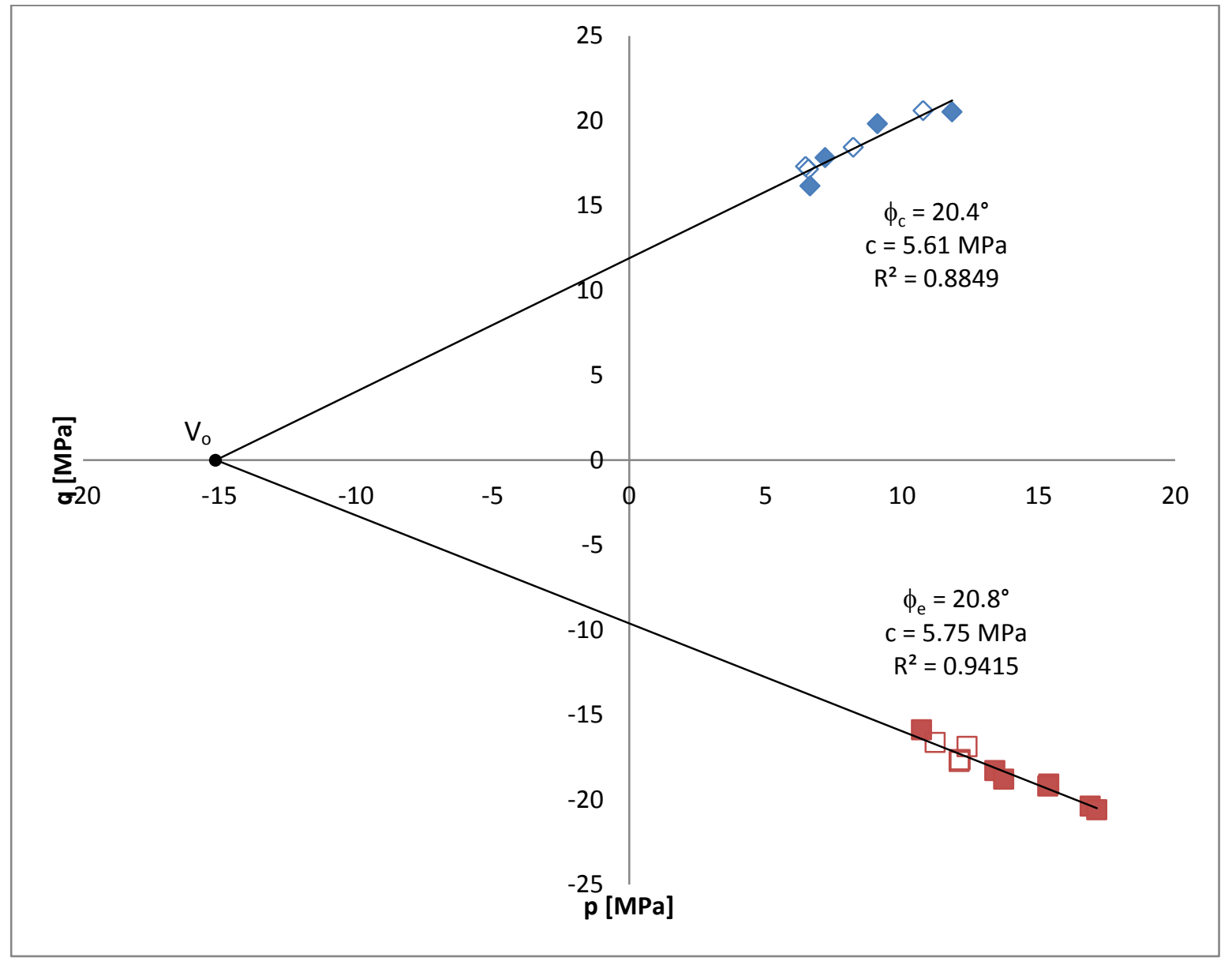

Figure 21. Triaxial compression and extension data with a common vertex. Open data points represent tests conducted in unloading stress path.

The angles of internal friction for compression and extension were calculated in the same manner as was shown for the unadjusted data. It was determined that $\phi_{c}=20.4^{\circ}$ and $\phi_{e}=20.8^{\circ}$. The 
compression cohesion, $c_{c}$, was calculated by first finding $b_{c}$ considering the slopes and vertex value, and then using equation (30):

$$
\begin{gathered}
b_{c}=\frac{6 c_{c} \cos \left(\phi_{c}\right)}{3-\sin \left(\phi_{c}\right)}=|-15.16(0.7855)|=11.91 \mathrm{MPa} \\
c_{c}=\frac{b_{c}\left(3-\sin \left(\phi_{c}\right)\right)}{6 \cos \left(\phi_{c}\right)}=5.61 \mathrm{MPa}
\end{gathered}
$$

Extension cohesion was calculated to be $c_{e}=5.75 \mathrm{MPa}$ using equation (31). Table 6 shows a comparison between calculated strength parameters for adjusted and unadjusted data.

Table 6. Strength parameters with common intercept $V_{o}$ (adjusted) and raw (unadjusted) data

\begin{tabular}{|l|c|c|c|c|}
\hline $\begin{array}{c}\text { Conventional triaxial } \\
\text { test method }\end{array}$ & $\begin{array}{c}\text { Unadjusted } \\
\text { data } \phi_{\mathrm{i}}\left[^{\circ}\right]\end{array}$ & $\begin{array}{c}\text { Adjusted } \\
\text { data } \phi_{\mathrm{i}}\left[^{\circ}\right]\end{array}$ & $\begin{array}{c}\text { Unadjusted data } \\
c_{i}[\mathrm{MPa}]\end{array}$ & $\begin{array}{c}\text { Adjusted data } \\
c_{i}[\mathrm{MPa}]\end{array}$ \\
\hline Compression, c & 19.7 & 20.4 & 5.71 & 5.61 \\
\hline Extension, e & 21.7 & 20.8 & 5.62 & 5.75 \\
\hline
\end{tabular}

When comparing the strength parameters calculated from the conventional triaxial compression and extension tests, a slight difference is observed. This difference is associated with an intermediate stress effect in the material. 


\subsection{Evaluation of failure criteria}

\subsection{Six-sided pyramidal failure surface}

\subsubsection{Formulation}

When analyzing any failure criterion it is useful to consider the geometrical representation in principal stress space. Any state of stress can be presented as a point with $\sigma_{1}, \sigma_{2}, \sigma_{3}$ coordinates, where $\sigma_{1}, \sigma_{2}, \sigma_{3}$ are the principal stresses with no order implied. The shape of the failure surface in principal stress space depends on the form of the failure criterion. For linear functions (e.g. MC), the surface will map as a series of six planes, each corresponding to a particular order of principal stresses, creating a hexagon with three-fold symmetry about the hydrostatic axis, $\sigma_{1}=\sigma_{2}=\sigma_{3}$. The six planes all pass through a common point known as the vertex $\left(V_{o}\right)$, which lies on the hydrostatic axis, and the failure surface has a pyramid shape (Figure 1).

The construction of the failure surface for an isotropic material in principal stress space is simplified greatly by the three-fold symmetry about the hydrostatic axis. Two points, which can be used with the vertex to construct the entire failure surface, can be determined through one cross-section along the hydrostatic axis, where two of the principal stresses are equal, e.g. $\sigma_{2}=$ $\sigma_{3}$ (Figure 22). This cross-section is also convenient because the surface can be defined from the data obtained during conventional triaxial testing in compression and extension. The two lines shown in the cross-section represent versions of equation (7) corresponding to different orders of principal stresses and define the intersection of two planes of the failure surface:

Compression

$\sigma_{1}=\sigma_{3} k_{p}+C_{o}$

Extension

$\sigma_{3}=\sigma_{1} k_{p}+C_{o} \quad$ or $\quad \sigma_{1}=\frac{\sigma_{3}}{k_{p}}-T_{o}$ 
where $T_{0}$ is the predicted $M C$ value of tensile strength. The compression line defines the intersection of the planes representing the principal stress orderings of $\sigma_{1}>\sigma_{2}>\sigma_{3}$ and $\sigma_{1}>\sigma_{3}>$ $\sigma_{2}$, while the extension line defines the intersection of the planes representing the principal stress orderings of $\sigma_{2}>\sigma_{3}>\sigma_{1}$ and $\sigma_{3}>\sigma_{2}>\sigma_{1}$.

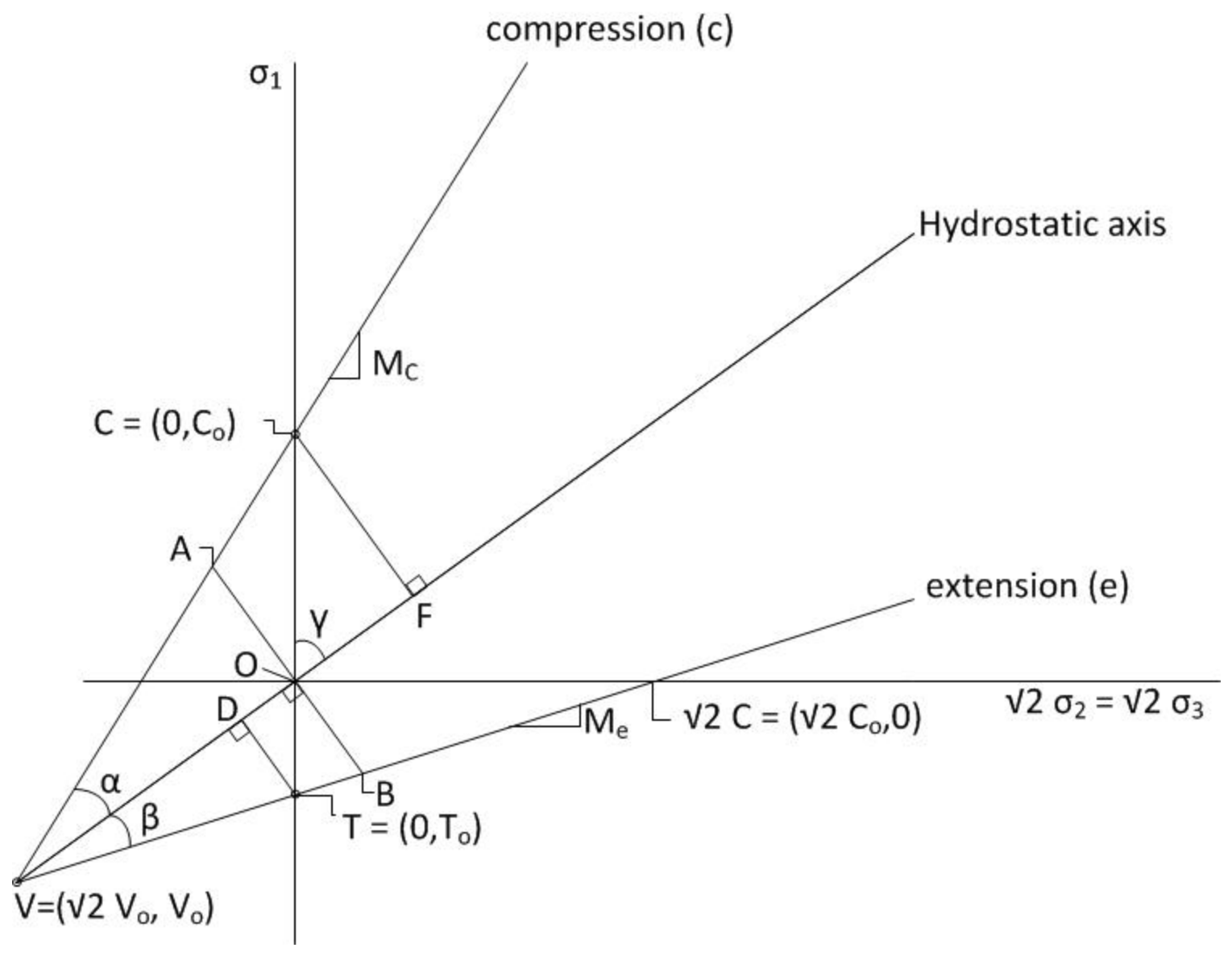

Figure 22. Cross-section of failure surface along hydrostatic axis corresponding to $\sigma_{2}=\sigma_{3}$.

The slopes $M_{c}$ and $M_{e}$ can be defined in terms of the strength parameter $\phi$ by differentiating equations (7.a) and (7.b), respectively:

$$
\begin{aligned}
& M_{c}=\frac{1}{\sqrt{2}} \frac{d \sigma_{1}}{d \sigma_{3}}=\frac{1}{\sqrt{2}} \frac{1+\sin \phi_{c}}{1-\sin \phi_{c}} \\
& M_{e}=\frac{1}{\sqrt{2}} \frac{d \sigma_{1}}{d \sigma_{3}}=\frac{1}{\sqrt{2}} \frac{1-\sin \phi_{e}}{1+\sin \phi_{e}}
\end{aligned}
$$


In order to draw any $\pi$-plane cross-section, only two lengths are required. The lengths of $O B$ and OA. From Figure 22,

$O B=O V \tan \beta$

where $O V=\sqrt{\left(V_{o}^{2}+V_{o}^{2}+V_{o}^{2}\right)}=\sqrt{3} V_{o}$. Next $\tan \beta$ can be determined from triangle TDV:

$\tan \beta=\frac{D T}{D V}=\frac{O T \sin \gamma}{O V-O D}=\frac{T_{o} \sin \gamma}{\sqrt{3} V_{o}-T_{o} \cos \gamma}$

where $\sin \gamma=\sqrt{\frac{2}{3}}$ and $\cos \gamma=\sqrt{\frac{1}{3}}$. Substituting this back into equation (37) gives

$\tan \beta=\frac{\sqrt{2} T_{o}}{3 V_{o}-T_{o}}$

Equation (38) can be substituted back into equation (36) to get

$O B=\frac{\sqrt{6} V_{o} T_{o}}{3 V_{o}-T_{o}}=\frac{\sqrt{6}}{\frac{3}{T_{o}}-\frac{1}{V_{o}}}$

Similarly,

$O A=O V \tan \alpha=\frac{\sqrt{6}}{\frac{3}{C_{o}}+\frac{1}{V_{o}}}$

These equations can be solved in terms of MC strength parameters by noting that the point $V$ is equivalent to

$-\sigma_{1}=-\sigma_{2}=-\sigma_{3}=V_{o}$

Substituting equation (41) into equation (7.a) or (7.b) gives

$V_{o}=V_{o} k_{p}+C_{o} \quad$ or $\quad V_{o}=\frac{C_{o}}{1-k_{p}}=-\frac{1-\sin \phi}{2 \sin \phi} C_{o}=-\frac{1+\sin \phi}{2 \sin \phi} T_{o}$

and $k_{p}=\frac{c_{o}}{T_{o}}$. By substituting equation (42) into equations (39) and (40)

$|O B|=\frac{2 \sqrt{6} V_{o} \sin \phi}{3+\sin \phi}$ 
$|O A|=\frac{2 \sqrt{6} V_{o} \sin \phi}{3-\sin \phi}$

With the lengths determined, the cross-sectional hexagon and six-sided pyramidal failure surface can be constructed (Figure 23.a).

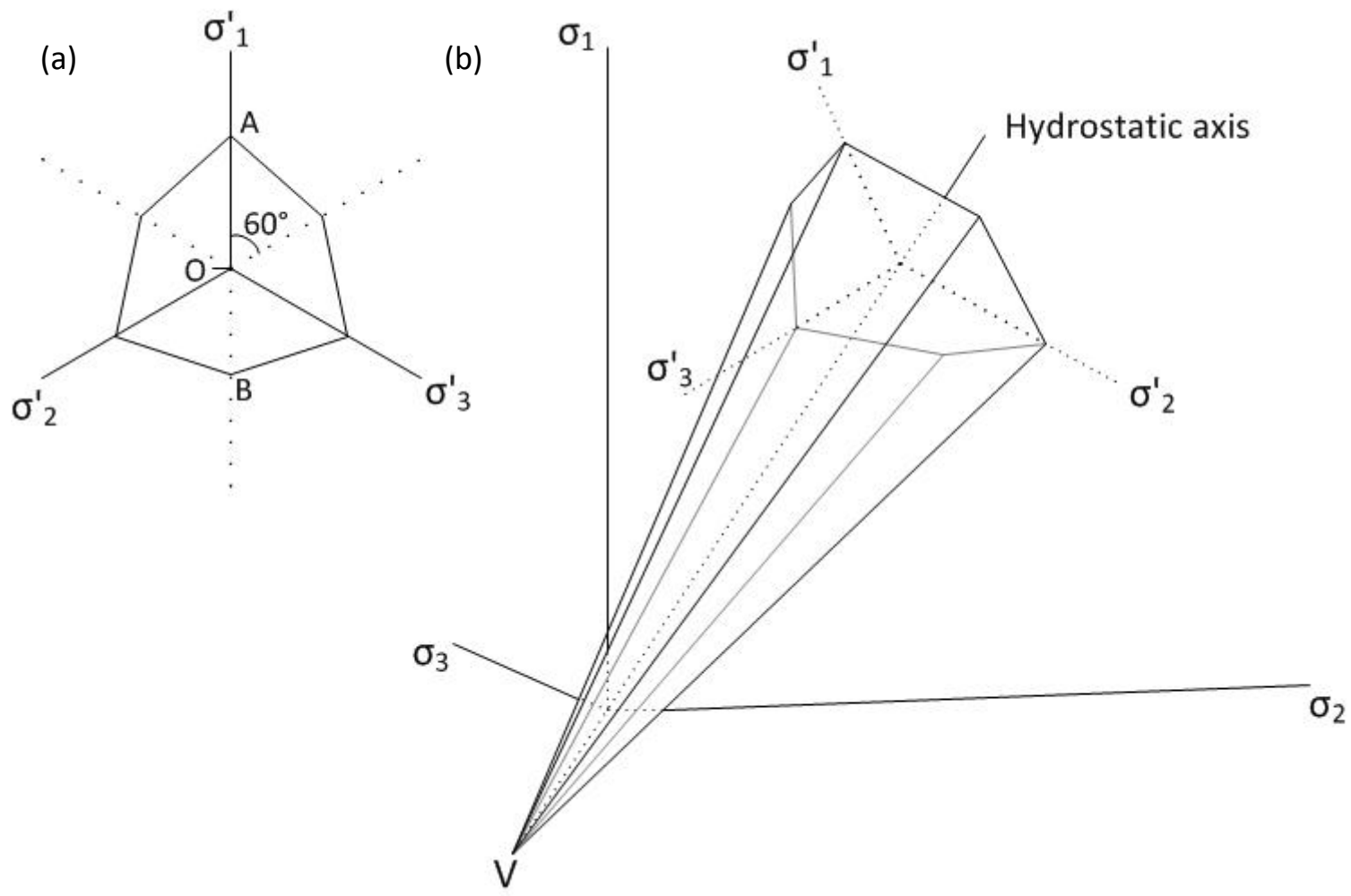

Figure 23. (a) $\pi$-plane cross-section at $\sigma_{1}=\sigma_{2}=\sigma_{3}=0$ plane, also known as the deviator plane. (b) Pyramidal surface in principal stress space.

Each of the six sides shown in Figure 23 (a) is an intersection of the equipressure plane with an oblique plane represented by an equation of the form

$A \sigma_{I}+B \sigma_{I I}+C \sigma_{I I I}=1$

Considering an isotropic material, any one of the sides of the pyramidal surface can be used to describe the rest. Therefore, only three constants, A, B, and C are necessary to define the six planes of the pyramidal surface (Figure 23.b). In order to determine each of these constants, three points on the plane must be known, e.g. $T_{o}, C_{o}$ and $V_{o}$ or $\phi_{\mathrm{c}}, \phi_{\mathrm{e}}$ and $V_{o}$. 


\subsubsection{Material parameters}

When performing a uniaxial compression test, the state of stress is $\sigma_{I}=C_{o}$ and $\sigma_{I I}=\sigma_{I I I}=0$, which leaves equation (45) as

$A=\frac{1}{C_{o}}$

When performing a uniaxial tension test, the state of stress is $\sigma_{I}=\sigma_{I I}=0$ and $\sigma_{I I I}=-T$, but the value predicted from the failure criterion will be used, which leaves equation (45) as

$C=-\frac{1}{T_{o}}$

Lastly, when failure occurs during uniform triaxial tension, the state of stress is $-V_{o}=\sigma_{I}=\sigma_{I I}=$ $\sigma_{I I I}$, which leaves equation (45) as

$A+B+C=\frac{1}{-V_{o}}$

Plugging equation (47) and (48) into equation (49) and rearranging gives

$B=-\frac{1}{V_{o}}-\frac{1}{C_{o}}+\frac{1}{T_{o}}$

It is convenient to write $C_{o}$ and $T_{o}$ in terms of $V_{o}, \mathrm{M}_{\mathrm{c}}$, and $\mathrm{M}_{\mathrm{e}}$. From Figure $17, C_{o}$ and $T_{o}$ can be represented as

$C_{o}=V_{o}\left(\sqrt{2} M_{c}-1\right)$

$T_{o}=V_{o}\left(1-\sqrt{2} M_{e}\right)$

which, when substituted into equation (50), gives

$B=-\frac{1}{V_{o}}-\frac{1}{V_{o}} \frac{1}{\left(\sqrt{2} M_{c}-1\right)}+\frac{1}{V_{o}} \frac{1}{\left(1-\sqrt{2} M_{e}\right)}$

After simplification, equation (53) can be written as 
$B=\frac{1}{V_{o}}\left[\frac{2 M_{c} M_{e}-1}{\left(\sqrt{2} M_{c}-1\right)\left(1-\sqrt{2} M_{e}\right)}\right]$

With the three constants solved for in terms of $V_{o}, M_{c}$ and $M_{e}$, the linear failure criterion can be written as

$\frac{1}{V_{o}} \frac{1}{\left(\sqrt{2} M_{c}-1\right)} \sigma_{I}+\frac{1}{V_{o}}\left[\frac{2 M_{c} M_{e}-1}{\left(\sqrt{2} M_{c}-1\right)\left(1-\sqrt{2} M_{e}\right)}\right] \sigma_{I I}+\frac{1}{V_{o}}\left[-\frac{1}{\left(1-\sqrt{2} M_{e}\right)}\right] \sigma_{I I I}=1$

A representation of equation (55) can be written in terms of strength parameters $\phi_{\mathrm{c}}$ and $\phi_{\mathrm{e}}$. For convenience, each constant will be analyzed individually. By substituting equation (34) into equation (55), the constant $A$ can be written as

$$
A=\frac{1}{V_{o}} \frac{1}{\left(\sqrt{2} M_{c}-1\right)}=\frac{1}{V_{o}} \frac{1}{\frac{1+\sin \phi_{c}}{1-\sin \phi_{c}}-1}=\frac{1}{V_{o}} \frac{1-\sin \phi_{c}}{2 \sin \phi_{c}}
$$

Similarly, by substituting equations (34) and (35) into equation (55), the constant B can be written in terms of $\phi_{\mathrm{c}}$ and $\phi_{\mathrm{e}}$ :

$$
B=\frac{1}{V_{o}}\left[\frac{2 M_{c} M_{e}-1}{\left(\sqrt{2} M_{c}-1\right)\left(1-\sqrt{2} M_{e}\right)}\right]=\frac{1}{V_{o}}\left[\frac{\left(\frac{1+\sin \phi_{c}}{1-\sin \phi_{c}}\right)\left(\frac{1-\sin \phi_{e}}{1+\sin \phi_{e}}\right)-1}{\left(\frac{1+\sin \phi_{c}}{1-\sin \phi_{c}}-1\right)\left(1-\frac{1-\sin \phi_{e}}{1+\sin \phi_{e}}\right)}\right]
$$

And after simplification, it can be written as

$B=\frac{1}{V_{o}}\left[\frac{\sin \phi_{c}-\sin \phi_{e}}{2 \sin \phi_{c} \sin \phi_{e}}\right]$

Lastly, the constant $\mathrm{C}$ can be written in terms of $\phi_{\mathrm{e}}$ if equation (35) is substituted into equation (55), giving

$$
C=-\frac{1}{V_{o}} \frac{1}{\left(1-\sqrt{2} M_{e}\right)}=-\frac{1}{V_{o}} \frac{1}{1-\frac{1-\sin \phi_{e}}{1+\sin \phi_{e}}}=-\frac{1}{V_{o}} \frac{1+\sin \phi_{e}}{2 \sin \phi_{e}}
$$

With the three constants defined in terms of strength parameters $\phi_{\mathrm{c}}$ and $\phi_{\mathrm{e}}$ and the vertex $V_{o}$, the linear failure criterion is

$$
\frac{1}{V_{o}}\left[\frac{1-\sin \phi_{c}}{2 \sin \phi_{c}}\right] \sigma_{I}+\frac{1}{V_{o}}\left[\frac{\sin \phi_{c}-\sin \phi_{e}}{2 \sin \phi_{c} \sin \phi_{e}}\right] \sigma_{I I}-\frac{1}{V_{o}}\left[\frac{1+\sin \phi_{e}}{2 \sin \phi_{e}}\right] \sigma_{I I I}=1
$$


which, if $\phi_{\mathrm{C}}=\phi_{\mathrm{e}}$, is equivalent to the MC failure criterion:

$\frac{1}{V_{o}}\left[\frac{1-\sin \phi}{2 \sin \phi}\right] \sigma_{I}-\frac{1}{V_{o}}\left[\frac{1+\sin \phi}{2 \sin \phi}\right] \sigma_{I I I}=1$

\subsubsection{Data analysis}

The results obtained during the conventional triaxial experiments are used to construct the sixsided pyramidal failure surface of the Calcarenite sample. The results of the triaxial experiments in terms of principal stress, $\sigma_{1}, \sigma_{2}, \sigma_{3}$ are given in Table 7 .

Table 7. Triaxial compression and extension results in terms of principal stresses $\sigma_{1}, \sigma_{2}$, and $\sigma_{3}$ (no order implied).

\begin{tabular}{|c|c|c|c|}
\hline Test & $\begin{array}{c}\text { Stress } \\
\text { Path }\end{array}$ & $\begin{array}{c}\sigma_{2}=\sigma_{3} \\
{[\mathrm{MPa}]}\end{array}$ & $\begin{array}{c}\sigma_{1} \\
{[\mathrm{MPa}]}\end{array}$ \\
\hline 4 & $\mathrm{~L}$ & 1.25 & 17.4 \\
\hline 8 & $\mathrm{~L}$ & 1.25 & 19.0 \\
\hline 9 & $\mathrm{~L}$ & 2.5 & 22.3 \\
\hline 10 & $\mathrm{~L}$ & 5.0 & 25.5 \\
\hline 16 & $\mathrm{U}$ & 2.1 & 20.5 \\
\hline 17 & $\mathrm{U}$ & 0.9 & 18.0 \\
\hline 18 & $\mathrm{U}$ & 3.90 & 24.5 \\
\hline 20 & $\mathrm{U}$ & 0.7 & 18.0 \\
\hline 15 & $\mathrm{~L}$ & 19.5 & 1.2 \\
\hline 21 & $\mathrm{~L}$ & 18.0 & 0.3 \\
\hline 22 & $\mathrm{~L}$ & 23.7 & 2.6 \\
\hline 23 & $\mathrm{~L}$ & 24.0 & 2.6 \\
\hline 24 & $\mathrm{~L}$ & 16.0 & 1.2 \\
\hline 25 & $\mathrm{~L}$ & 16.0 & 0.1 \\
\hline 5 & $\mathrm{U}$ & 16.8 & 0.1 \\
\hline 6 & $\mathrm{U}$ & 18.0 & 1.1 \\
\hline 7 & $\mathrm{U}$ & 21.8 & 2.7 \\
\hline 12 & $\mathrm{U}$ & 18.0 & 0.4 \\
\hline 13 & $\mathrm{U}$ & 21.8 & 2.5 \\
\hline
\end{tabular}


The triaxial compression and extension tests performed on the Calcarenite sample were all performed with the axial direction of the core being in the $\sigma_{1}$ direction (along the $z$-axis). This allows for the locus of the pyramidal failure surface corresponding to $\sigma_{2}=\sigma_{3}$ to be constructed from the data presented in Table 7. Another important property that was calculated was the point where the failure surfaces intersect, $V_{o}$. From the $p-q$ plot, this point was determined to occur at $\sigma_{1}=\sigma_{2}=\sigma_{3}=-V_{o}=-15.16 \mathrm{MPa}$. The resulting locus is presented if Figure 24. Note the $\mathrm{x}$-axis is equivalent to $\sqrt{2} \sigma_{2}=\sqrt{2} \sigma_{3}$ while the values presented in Table 7 represent $\sigma_{2}=\sigma_{3}$.

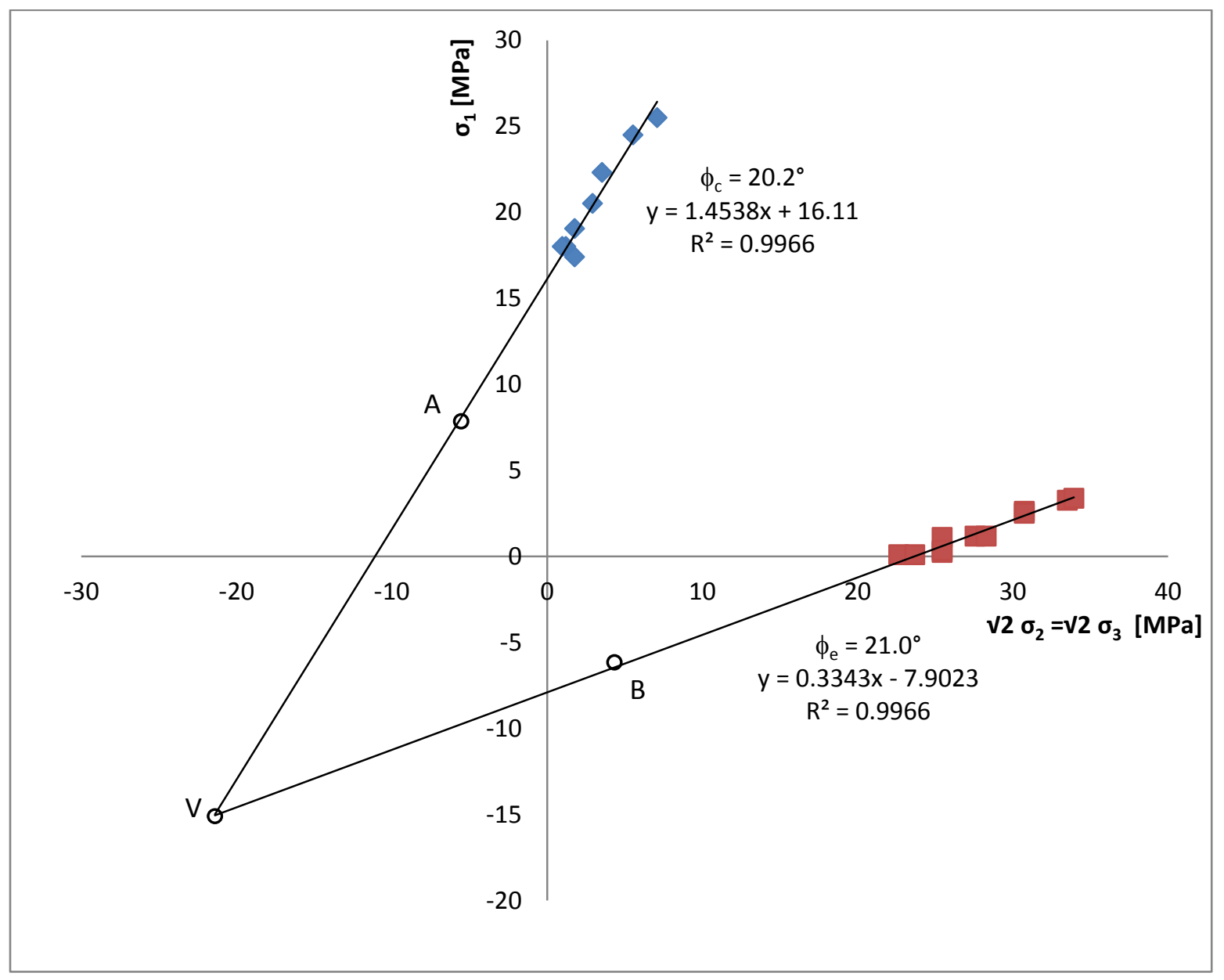

Figure 24. Failure locus corresponding to $\sigma_{2}=\sigma_{3}$. Open circles were extrapolated from the data.

The angles of internal friction for compression and extension of Calcarenite are determined using the slopes of the lines of best fit and equations (34) and (35), with an intersection of $V$. 


$$
\begin{gathered}
M_{c}=\frac{1+\sin \phi_{c}}{\sqrt{2}\left(1-\sin \phi_{c}\right)}=1.4538 \rightarrow \phi_{c}=20.2^{\circ} \\
M_{e}=\frac{1-\sin \phi_{e}}{\sqrt{2}\left(1+\sin \phi_{e}\right)}=0.3343 \rightarrow \phi_{e}=21.0^{\circ}
\end{gathered}
$$

These values closely agree with the values of $\phi_{c}=20.4^{\circ}$ and $\phi_{e}=20.8^{\circ}$ that were calculated from the $p-q$ plot (Figure 19). The fact that $\phi_{\mathrm{c}}$ is different than $\phi_{\mathrm{e}}$ suggests that there is an intermediate stress effect for the Calcarenite sample. The different angles of internal friction $\phi_{c}$ and $\phi_{\mathrm{e}}$ change the shape of the failure surface. Figure 25 shows, in dashed lines, cross sections of the MC deviatoric plane $\left(\sigma_{1}=\sigma_{2}=\sigma_{3}=0\right)$ of failure surfaces representing $\phi_{c}=20.2^{\circ}$ and $\phi_{e}=21.0^{\circ}$. The solid line represents the cross section of the linear failure surface for Calcarenite. The six-sided pyramidal failure surface can also be constructed with the measured data (Figure 26).

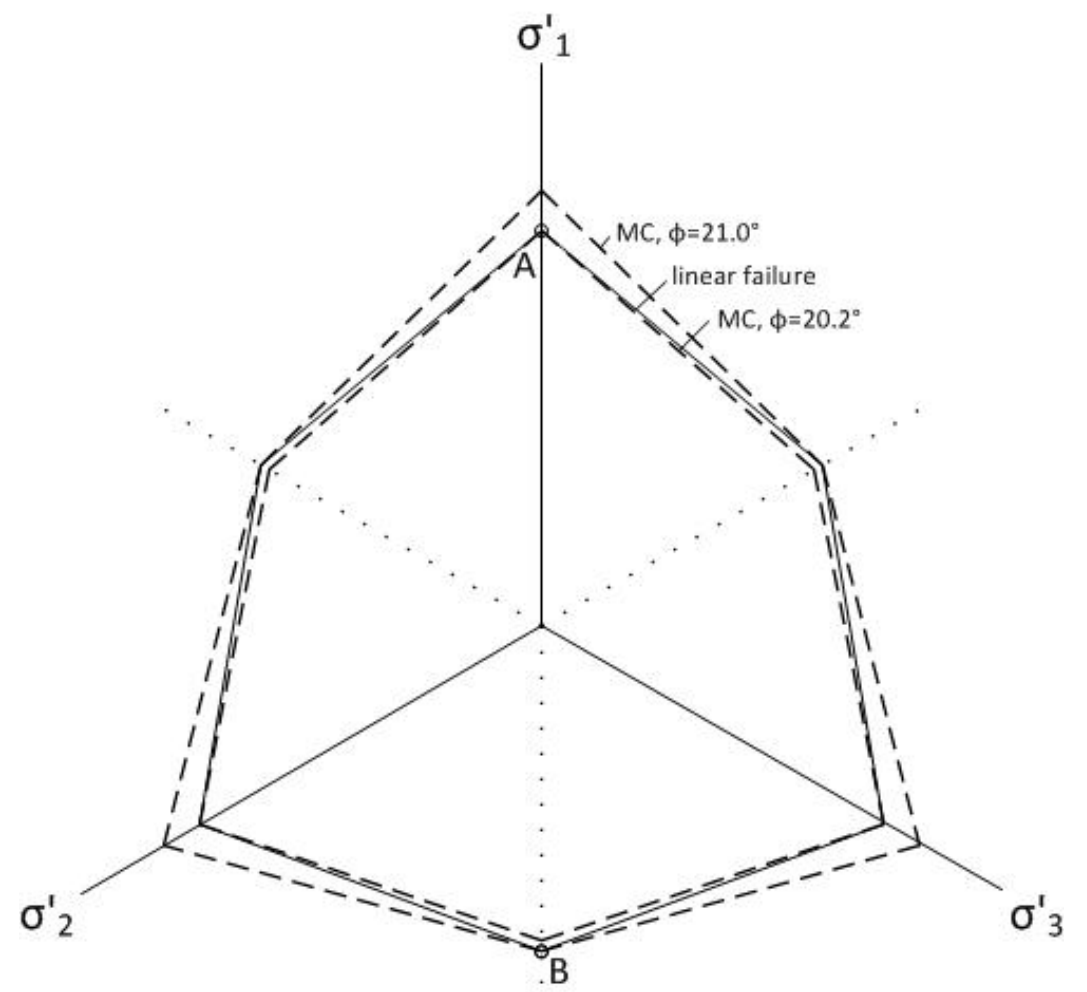

Figure 25. Comparison of cross section of MC failure surfaces and linear failure surface with intermediate stress effect. 


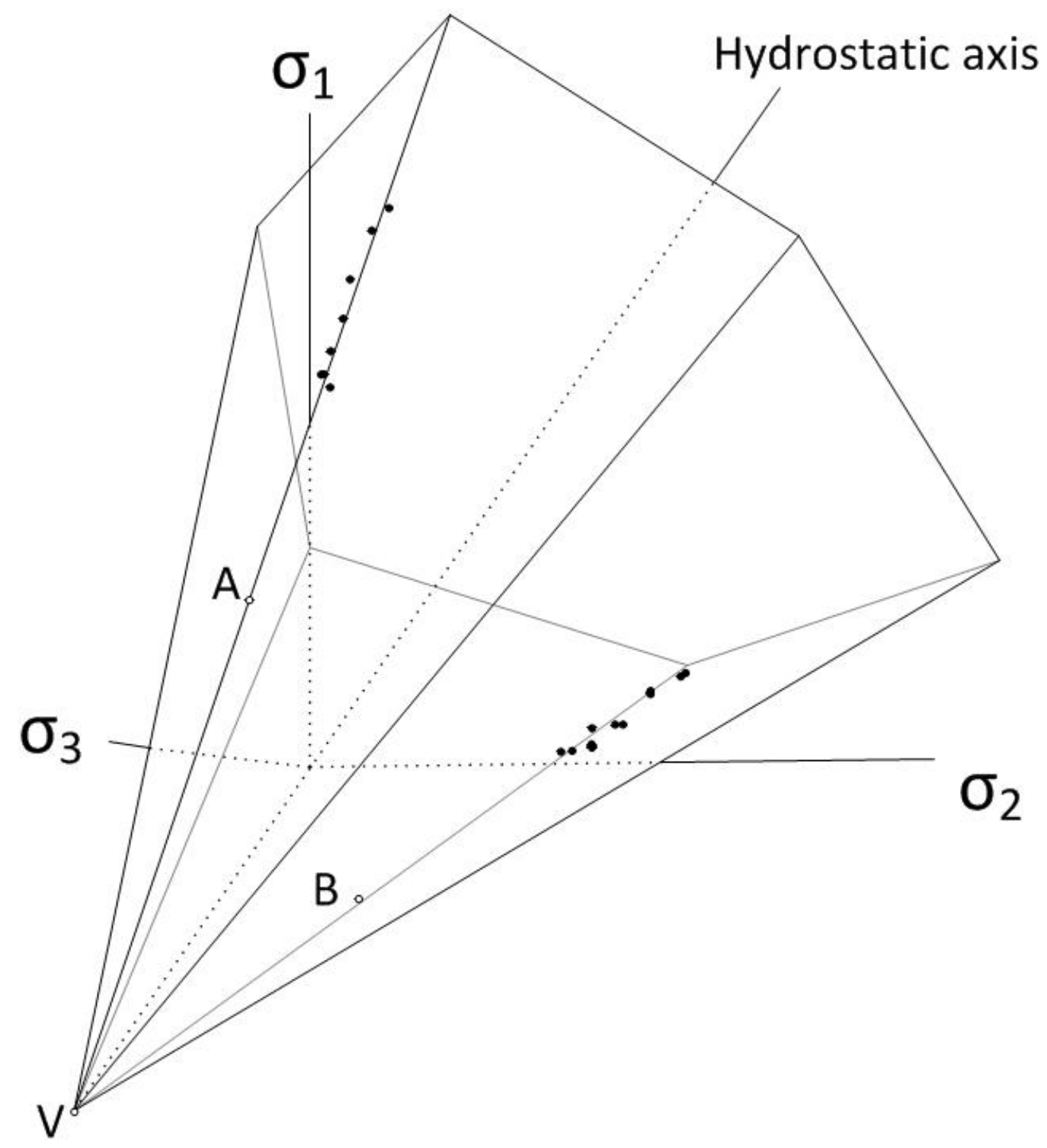

Figure 26. Experimental data points plotted on pyramidal failure surface. Open circles are extrapolated from data.

The constants in the equation for the six planes, equation (60), can be determined from the strength parameters $\phi_{\mathrm{c}}$ and $\phi_{\mathrm{e}}$ and vertex $V_{o}$ determined from testing: $V_{o}=15.16 \mathrm{MPa}, \phi_{c}=$ $20.2^{\circ}$ and $\phi_{e}=21.0^{\circ}$, and the linear failure criterion is

$$
0.062 \sigma_{I}-0.02 \sigma_{I I}-0.126 \sigma_{I I I}=1
$$


compared to the MC failure criterion assuming $\phi_{\mathrm{c}}=20.6^{\circ}$ (average of $\phi_{\mathrm{c}}$ and $\phi_{\mathrm{e}}$ )

$$
0.061 \sigma_{I}-0.127 \sigma_{I I I}=1
$$

\subsection{Tension cut-off}

An issue with any shear failure criterion is its inability to correctly predict the tensile strength of the material, essentially because the failure criterion predicts the tensile strength of a material assuming shear failure. However, it is known that brittle materials fail on planes normal to the direction of tensile stress, which would suggest the mode of failure to be tensile as opposed to shear Therefore, the failure plane is perpendicular to $\sigma_{\| I I}=-T$, where $T$ is the tensile strength of the material (Figure 27).

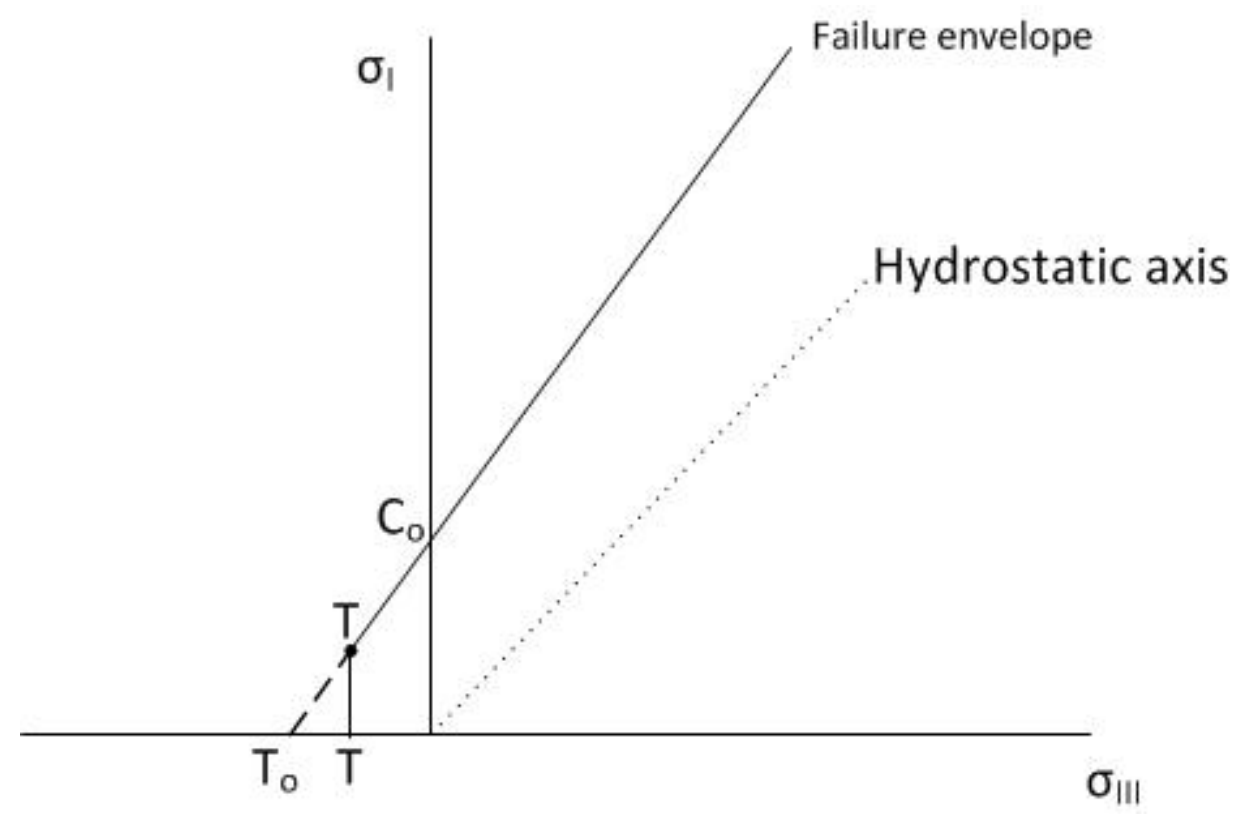

Figure 27. Failure envelope in principal stress plane, $\sigma_{I}-\sigma_{\| I}$.

Paul [1961] suggested the concept of a tension cut-off and a modified MC failure criterion that would account for this transition from shear to tensile failure modes. Paul assumed that a brittle material will fail according to the values predicted by equation ( 7 ) 
$\sigma_{I}=\sigma_{I I I} K_{p}+C_{o}$

when $\sigma_{\| I I}>-T$, otherwise

$\sigma_{I I I}=-T$

The modified MC failure criterion can also be represented in principal stress space. Following the same construction processes presented for the six-sided pyramidal failure surface, the crosssection of the pyramidal surface along the hydrostatic axis with two principal stresses equal is shown in Figure 28.

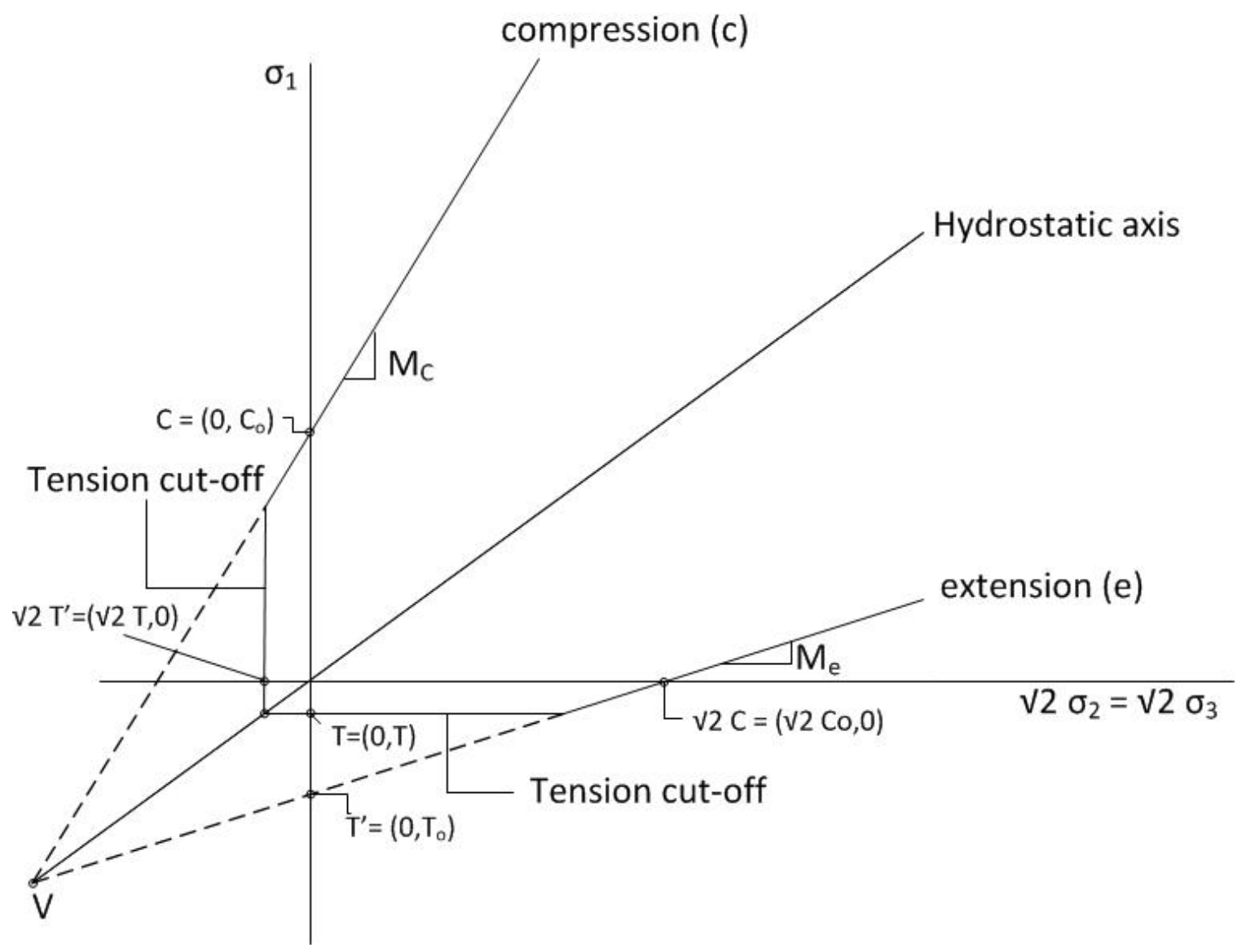

Figure 28. Cross-section of failure surface along hydrostatic axis corresponding to $\sigma_{2}=\sigma_{3}$ with tension cut-offs included. 
All equations previously presented for the determination of the six-sided pyramidal failure surface hold true for the development of the six-sided pyramidal failure surface with tension cut-offs. The only difference is that this pyramid is intercepted by a second pyramid with three planes perpendicular to the principal stress axes (Figure 29).

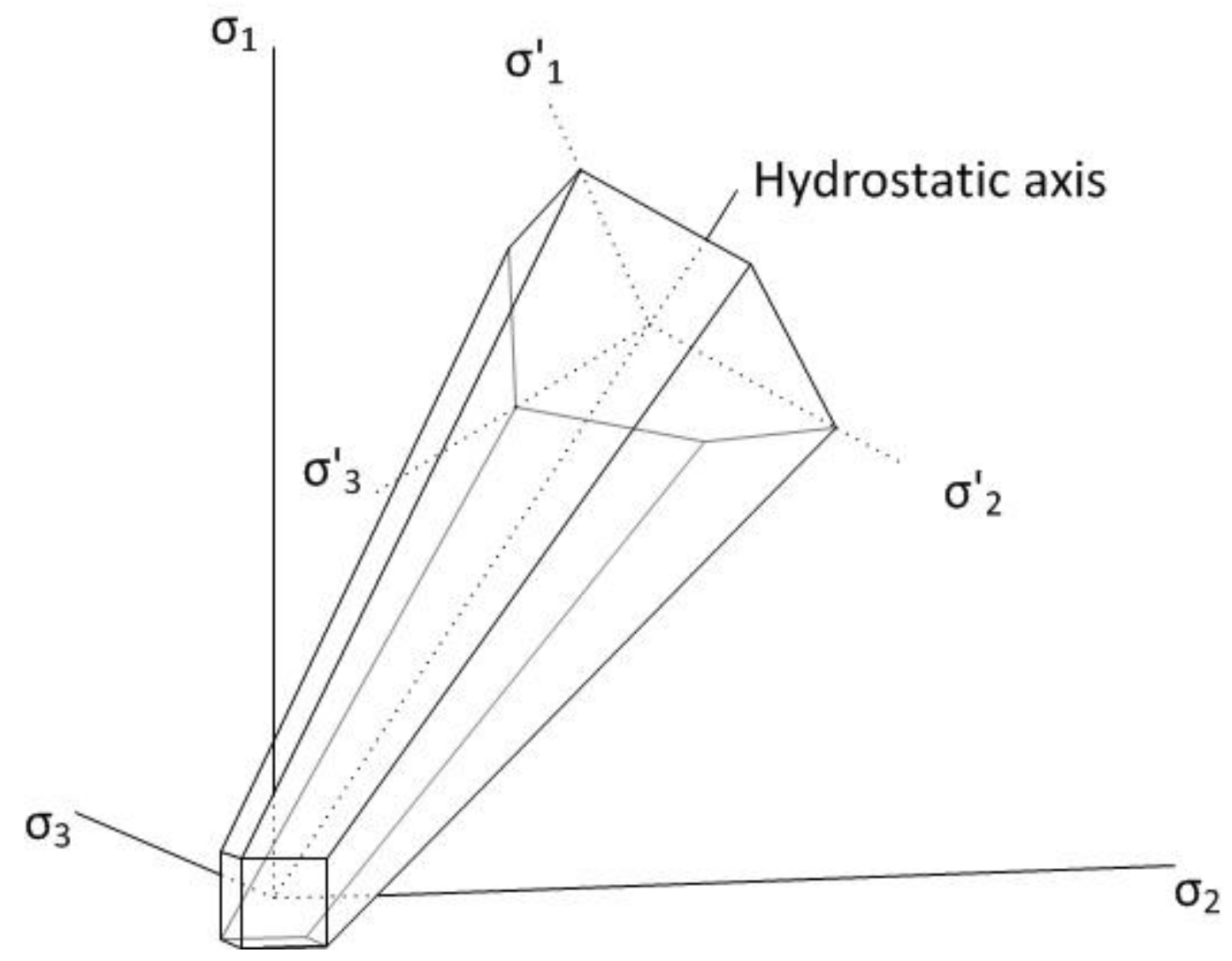

Figure 29. Pyramidal failure surface in principal stress space with tension cut-offs.

From the uniaxial tension test performed, it was determined that $T=2.06 \mathrm{MPa}$. With this result, the tension cut-off for the Calcarenite sample tested could be incorporated. The cross-section of the failure surface, including the data and constraint, along the hydrostatic axis with two principal stresses equal, is shown in Figure 30. 


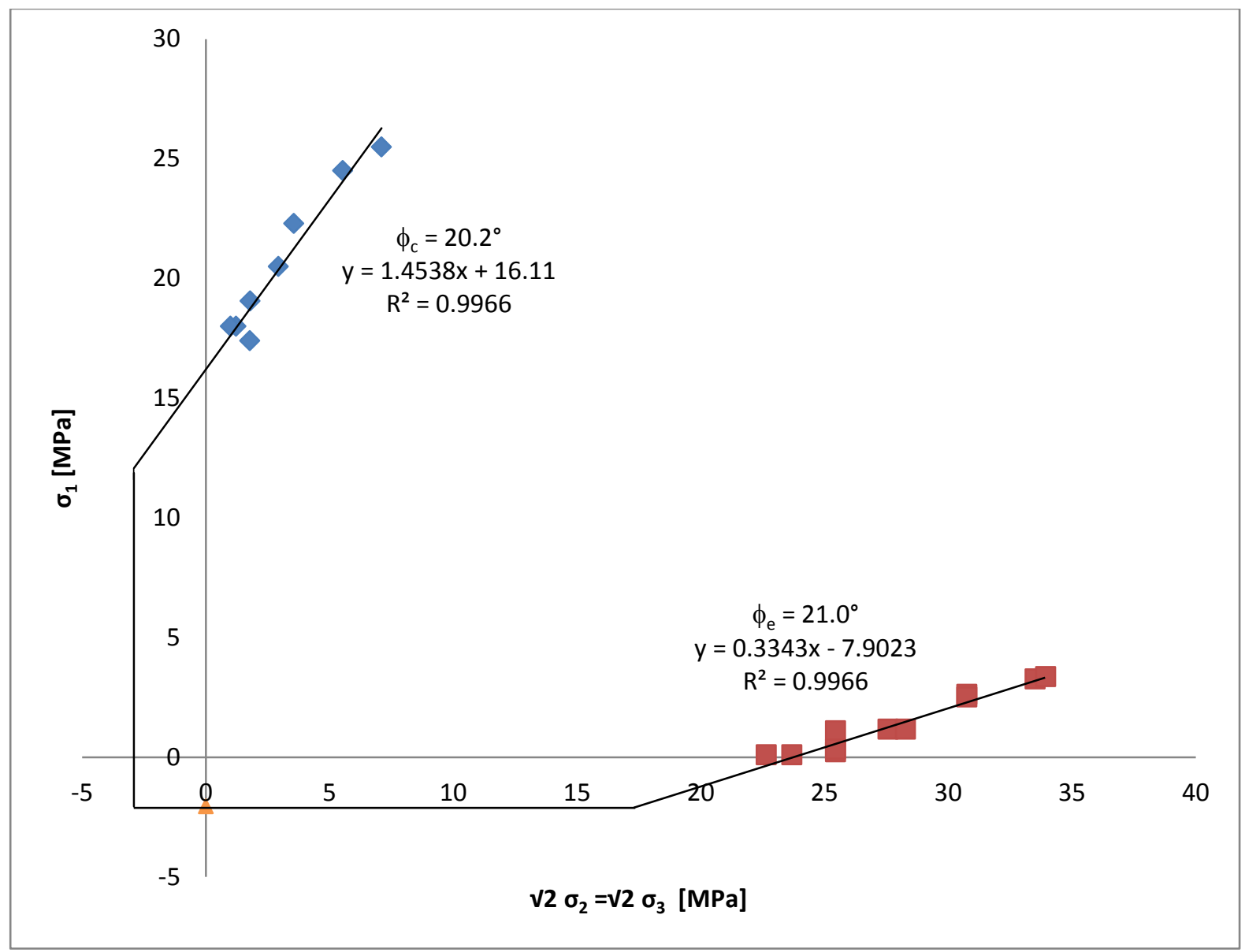

Figure 30. Cross-section of failure surface along hydrostatic axis corresponding to $\sigma_{2}=\sigma_{3}$ with tension cut-offs included.

The tension cut-offs can also be incorporated to the failure surface in principal stress space. Figure 31 shows the six-sided pyramidal failures surface including tension cut-offs calculated from the direct tension test. 


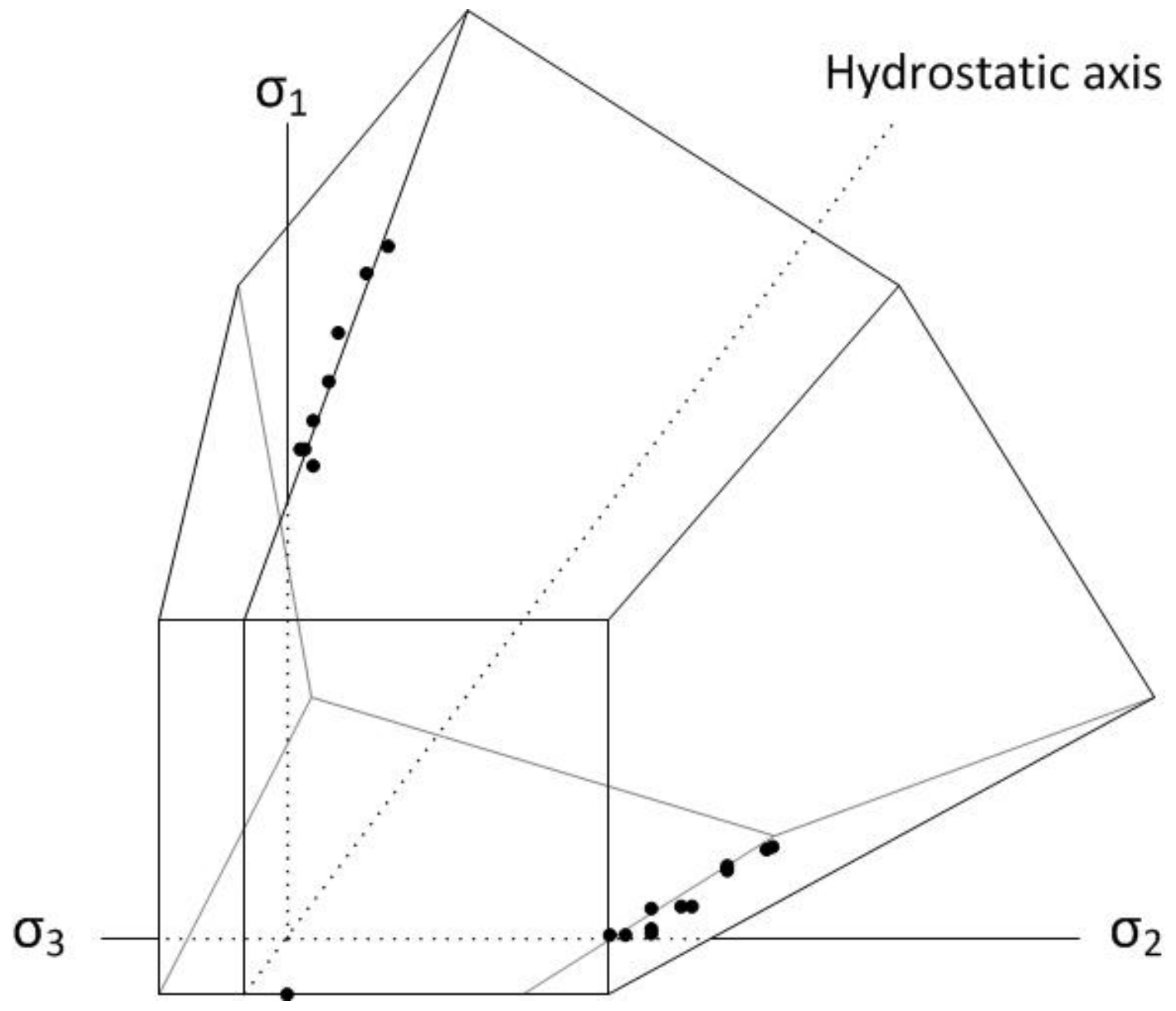

Figure 31. Pyramidal failure surface in principal stress space with tension cut-offs.

\subsection{Twelve-sided pyramidal failure surface}

The conventional triaxial experiments showed that the intermediate stress did affect the material properties determined for the Calcarenite. To examine other stress states besides axisymmetric compression and extension, two plane-strain (multi-axial) experiments were performed at lateral (confining) stress $\sigma_{\mathrm{II}}=2.0$ and $3.0 \mathrm{MPa}$. The specimen dimensions and results are presented in Table 8. The data from the test with $p=11.2 \mathrm{MPa}$ are plotted on an equipressure plane of the sixsided pyramidal section (Figure 32), and it is clear that the failure surface does not capture the observed response. 
Table 8. Plane-strain experimental results

\begin{tabular}{|c|c|c|c|c|c|c|c|}
\hline Specimen & $\begin{array}{c}\text { Width } \\
{[\mathrm{mm}]}\end{array}$ & $\begin{array}{c}\text { Height } \\
{[\mathrm{mm}]}\end{array}$ & $\begin{array}{c}\text { Thickness } \\
{[\mathrm{mm}]}\end{array}$ & $\begin{array}{c}\text { Axial } \\
\text { stress, } \sigma_{\text {I }} \\
{[\mathrm{MPa}]}\end{array}$ & $\begin{array}{c}\text { Lateral } \\
\text { stress, } \sigma_{\text {III }} \\
{[\mathrm{MPa}]}\end{array}$ & $\begin{array}{c}\text { Intermediate } \\
\text { stress, } \sigma_{\text {III }} \\
{[\mathrm{MPa}]}\end{array}$ & $\begin{array}{c}\text { Mean } \\
\text { stress, } p \\
{[\mathrm{MPa}]}\end{array}$ \\
\hline Bx-1 & 100.47 & 74.93 & 27.98 & 27.4 & 3.0 & 7.7 & 12.7 \\
\hline Bx-2 & 100.69 & 99.8 & 41.91 & 23.6 & 2.0 & 8.0 & 11.2 \\
\hline
\end{tabular}

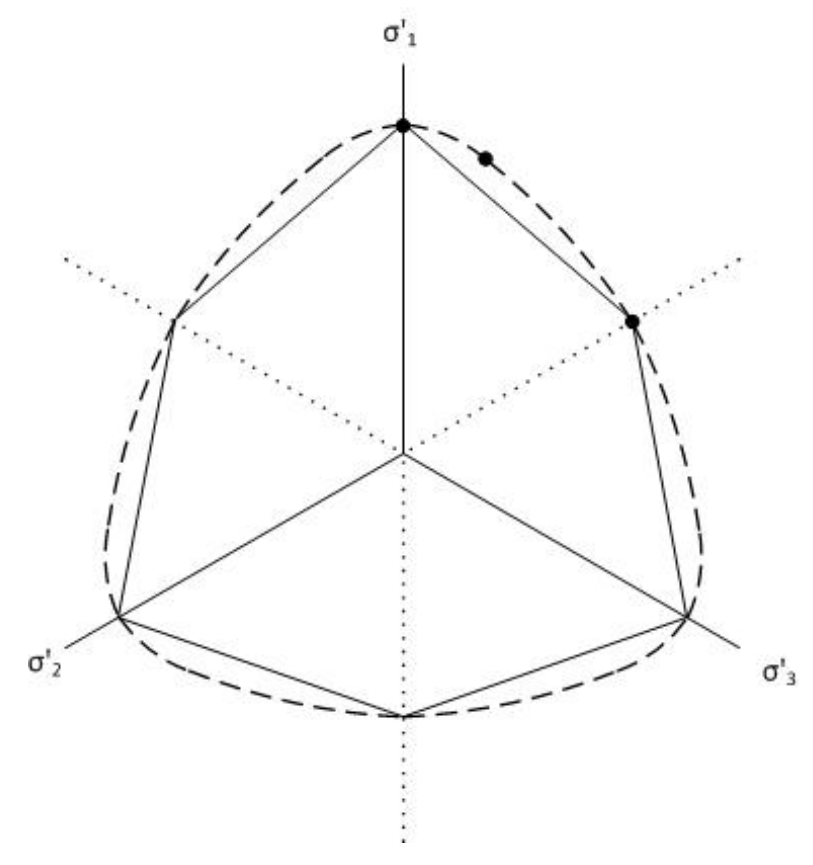

Figure 32. Equipressure plane of $p=11.2 \mathrm{MPa}$ with plane-strain data point.

To better approximate the actual behavior, the six-sided surface must be modified. An approach suggested by Matsuoka and Nakai [1974], Willam and Warnke [1974], and others is to fit a curve to the data (shown as dashed line in Figure 32), creating a smooth failure surface. To keep retain mathematical simplicity, Paul [1968c] suggested a piecewise linear description of the curved failure surface. By using a sufficient number of planes, a nonlinear failure surface can be accurately approximated (Figure 33). 


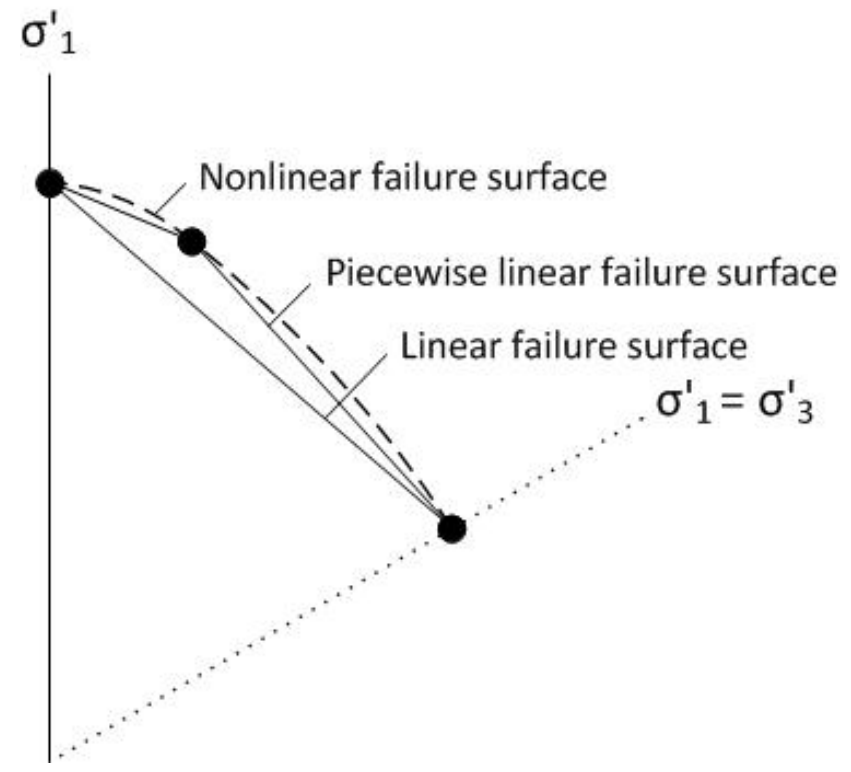

Figure 33. Section showing nonlinear, piecewise linear, and linear failure surfaces.

By incorporating the data point obtained from plane-strain testing, a piecewise linear surface consisting of twelve sides can be constructed (Figure 34). The twelve sides in Figure 34 are similar to the six sides of Figure 21.(a) in the fact that each side can be represented by equation (45).

The constants of equation (45) are determined by realizing the twelve-sided equipressure crosssection is the intersection of two hexagons, with the portion of the hexagon closer to the hydrostatic axis controlling failure, shown as the solid line in Figure 34. By considering each hexagon separately, all twelve sides of the surface can be described with six constants, three for each hexagon. Considering the hexagon with vertices $D_{1}{ }^{1}, D_{2}{ }^{1}, D_{3}{ }^{1}$ in Figure 34 , equation (45) can be written as

$A^{(1)} \sigma_{I}+B^{(1)} \sigma_{I I}+C^{(1)} \sigma_{I I I}=1$

Similarly, the hexagon with vertices $D_{1}{ }^{2}, D_{2}{ }^{2}, D_{3}{ }^{2}$, can be represented as

${ }^{(2)} \sigma_{I}+B^{(2)} \sigma_{I I}+C^{(2)} \sigma_{I I I}=1$ 


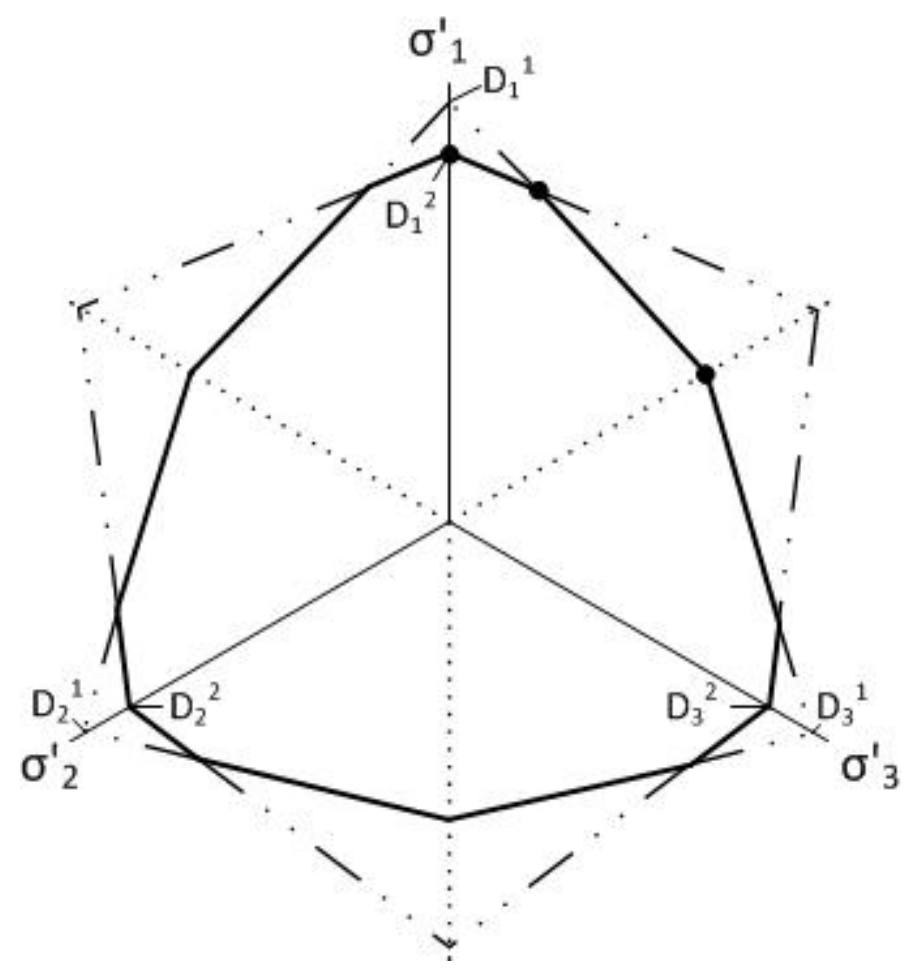

Figure 34. Cross-section of equipressure plane of a twelve-sided piecewise linear failure surface at $p=11.2 \mathrm{MPa}$.

As shown in Figure 20, if $V_{o}, C_{o}$, and $T_{o}$ are known, the constants can be solved in terms of compression and extension friction angles. However, Figure 35 shows that $C_{o}^{(1)}$ and $T_{o}^{(2)}$ cannot be measured through testing, because the failure surface dictates failure before reaching these values. The twelve-sided pyramidal failure surface in principal stress space with a common vertex $\left(\mathrm{V}_{0}{ }^{(1)}=\mathrm{V}_{0}{ }^{(2)}\right)$ is shown in Figure 36. 


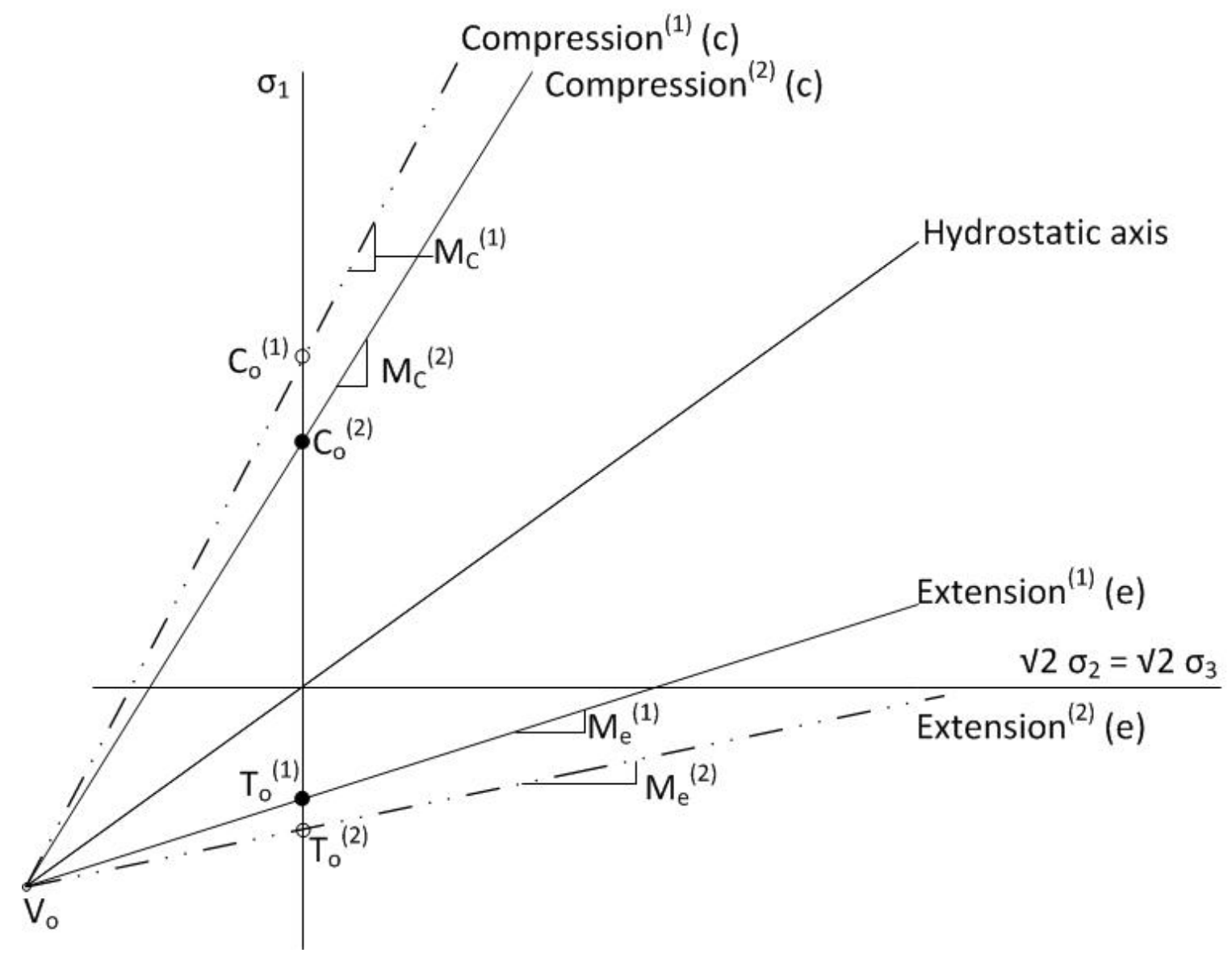

Figure 35. Cross-section of failure surface along hydrostatic axis corresponding to $\sigma_{2}=\sigma_{3}$. Open circles are extrapolated from data. 


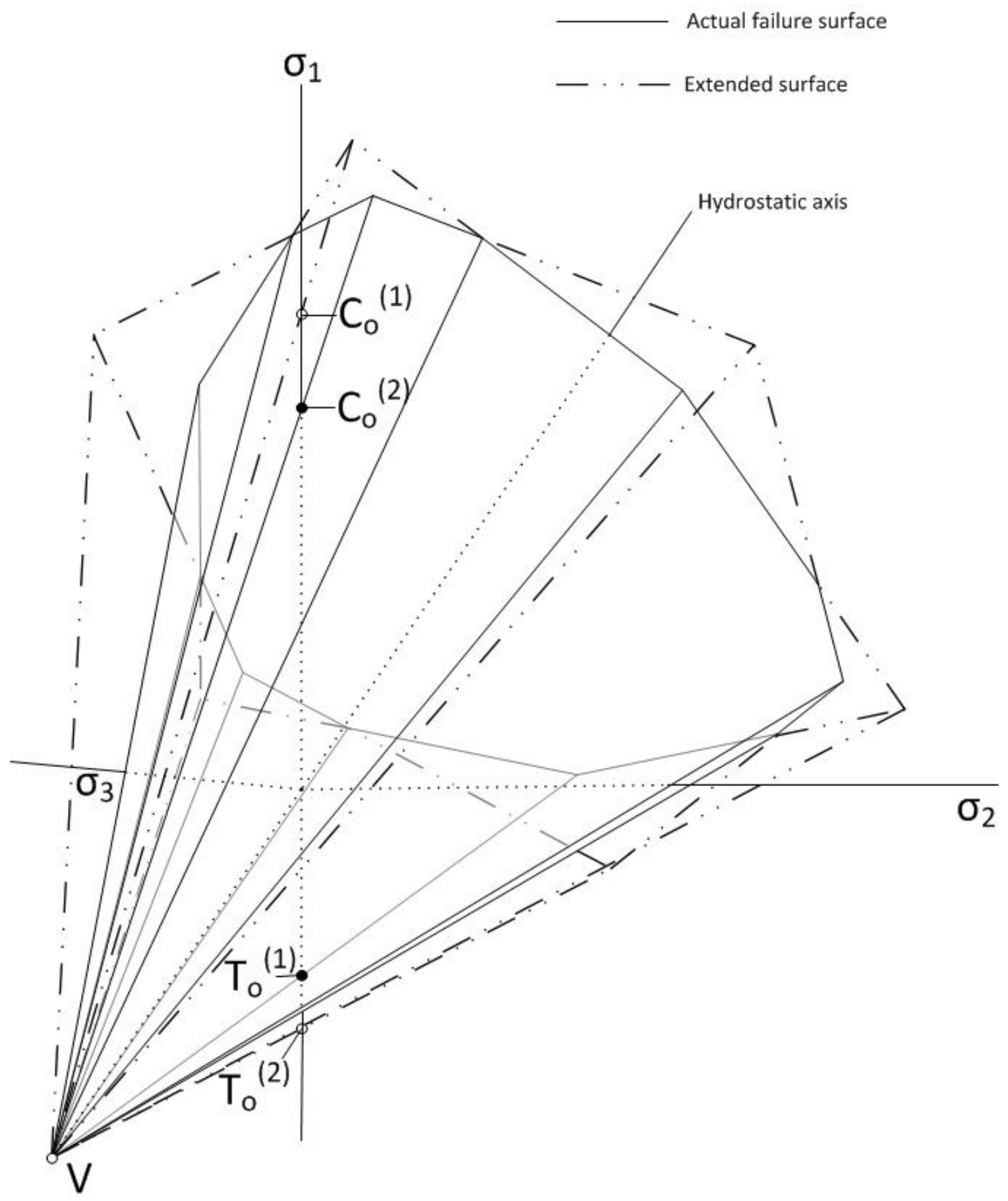

Figure 36. Twelve-sided pyramidal failure surface in principal stress space with theoretical failure surface shown. Open circles are extrapolated from data. 
To determine the fictitious friction angles, the failure surface must be extrapolated from the intersection, taken as the plane-strain point, of the two hexagons and the value of $C_{0}^{(2)}$ and $T_{0}^{(1)}$. Figure 37 shows a $60^{\circ}$ section of an equipressure plane plotted in the $\sigma_{F}-\sigma_{E}$ coordinate system [Davis and Selvadurai 2002], where

$\sigma_{E}=\frac{1}{\sqrt{2}}\left(-\sigma_{2}+\sigma_{3}\right)$

and

$\sigma_{F}=\frac{1}{\sqrt{6}}\left(2 \sigma_{1}-\sigma_{2}-\sigma_{3}\right)$

Note that the deviator stress $q$ is related to $\sigma_{F}$ and $\sigma_{E}$ :

$\sqrt{\frac{2}{3}} q=\sqrt{\left(\sigma_{E}^{2}+\sigma_{F}^{2}\right)}$

The points $\left(\sigma_{E, c}{ }^{(1)}, \sigma_{F, c}{ }^{(1)}\right),\left(\sigma_{E, e}{ }^{(2)}, \sigma_{F, e}{ }^{(2)}\right)$, and $\left(\sigma_{E, b}, \sigma_{F, b}\right)$ are the points determined from triaxial compression, triaxial extension and plane-strain (multi-axial) experiments. The points $\left(\sigma_{\mathrm{E}, \mathrm{c}}{ }^{(2)}, \sigma_{\mathrm{F}, \mathrm{c}}{ }^{(2)}\right)$ and $\left(\sigma_{\mathrm{E}, \mathrm{e}}{ }^{(1)}, \sigma_{\mathrm{F}, \mathrm{e}}{ }^{(1)}\right)$ are the points that need to be determined using the following equations:

$$
\begin{aligned}
& y^{(1)}=\frac{\left(\sigma_{F, b}-\sigma_{F, c}{ }^{(1)}\right)}{\left(\sigma_{E, b}-\sigma_{E, c}(1)\right)} \sigma_{E}+\sigma_{F, c}{ }^{(1)} \\
& y^{(2)}=\frac{\left(\sigma_{F, c}{ }^{(2)}-\sigma_{F, b}\right)}{\left(\sigma_{E, c}{ }^{(2)}-\sigma_{E, b}\right)} \sigma_{E}+\sigma_{F, c}{ }^{(2)} \\
& y^{(3)}=\tan \left(30^{\circ}\right) \sigma_{E}=0.5775 \sigma_{E}
\end{aligned}
$$

Once these points are known, the relation to the deviator stress associated with each stress state can be calculated using equation (67). 


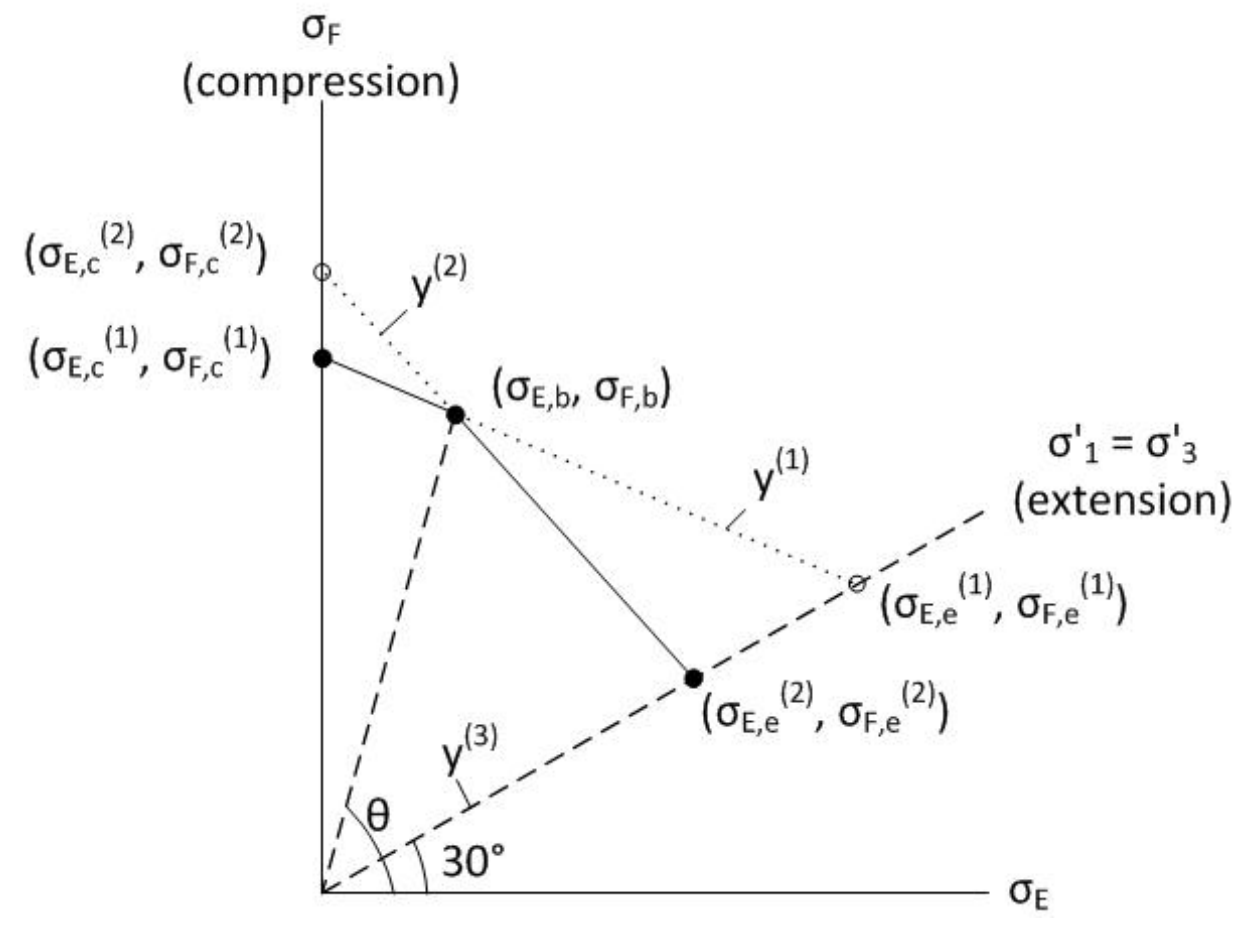

Figure 37. Section of an equipressure plane plotted in the $\sigma_{F}-\sigma_{E}$ coordinate system. Open circles are extrapolated from data.

Another important value that can be determined from Figure 37 is the Lode angle $\theta$, which is related to the principal stresses [Davis and Selvadurai 2002]:

$\tan \theta=\frac{\sigma_{F}}{\sigma_{E}}=\frac{2 \sigma_{1}-\sigma_{2}-\sigma_{3}}{\sqrt{3}\left(\sigma_{3}-\sigma_{2}\right)}$

The Lode angle $\theta$ is a convenient method for determining which pyramidal surface is controlling the failure of the specimen. By calculating $\theta$ from the multi-axial experiment, a range for which each pyramidal surface is describing the failure surface can be calculated.

Considering the data points obtained at a mean stress of $p=11.2 \mathrm{MPa}$, the deviator stress associated with the unknown points $\left(\sigma_{\mathrm{E}, \mathrm{c}}{ }^{(2)}, \sigma_{\mathrm{F}, \mathrm{c}}{ }^{(2)}\right)$ and $\left(\sigma_{\mathrm{E}, \mathrm{e}}{ }^{(1)}, \sigma_{\mathrm{F}, \mathrm{e}}{ }^{(1)}\right)$ can be calculated. From triaxial compression and extension testing, points $\left(\sigma_{\mathrm{E}, \mathrm{c}}{ }^{(1)}, \sigma_{\mathrm{F}, \mathrm{c}}{ }^{(1)}\right)$ and $\left(\sigma_{\mathrm{E}, \mathrm{e}}{ }^{(2)}, \sigma_{\mathrm{F}, \mathrm{e}}{ }^{(2)}\right)$ are $(0,16.89)$ and $(11.75,6.79)$ respectively, while point $\left(\sigma_{\mathrm{E}, \mathrm{b}}, \sigma_{\mathrm{F}, \mathrm{b}}\right)$, determined from the plane-strain experiment is $(4.19,15.14)$, which gives a Lode angle $\theta=74.5^{\circ}$. Using these data points $\left(\sigma_{E, c}{ }^{(2)}, \sigma_{F, c}{ }^{(2)}\right)$ and 
$\left(\sigma_{E, e}{ }^{(1)}, \sigma_{F, e}{ }^{(1)}\right)$ were determined to be $(0,19.77)$ and $(16.97,9.80)$. From equation (67), the relation to the deviator stress associated with each point can be determined:

$$
\begin{gathered}
\sqrt{\frac{2}{3}} q=\sqrt{\left(0^{2}+19.77^{2}\right)}=19.77 \\
\sqrt{\frac{2}{3}} q=\sqrt{\left(16.97^{2}+9.80^{2}\right)}=19.60
\end{gathered}
$$

Once the relation to the deviator stress is determined for the two unknown points, the values of $C_{o}^{(1)}$ and $T_{o}^{(2)}$ can be obtained by using one of two approaches: (1) assume both pyramids have the same vertex; (2) assume each pyramid has a separate vertex.

1. If $V_{o}$ is assumed to be the same for both hexagons, then all twelve sides of the failure surface can be defined with five constants; $C_{o}^{(1)}, C_{o}^{(2)}, T_{o}^{(1)}, T_{o}^{(2)}$, and $V_{o}$. The unknown values of $C_{0}^{(1)}$ and $T_{o}^{(2)}$ can be determined using slightly modified forms of equations (36) and (40):

$$
\begin{aligned}
& \sqrt{\frac{2}{3}} q=\left(\sqrt{3}\left|V_{o}\right|+\sqrt{3} p\right) \tan \alpha=\left(\sqrt{3}\left|V_{o}\right|+\sqrt{3} p\right) \frac{\sqrt{2} C_{o}}{3\left|V_{o}\right|+C_{o}} \\
& \sqrt{\frac{2}{3}} q=\left(\sqrt{3}\left|V_{o}\right|+\sqrt{3} p\right) \tan \beta=\left(\sqrt{3}\left|V_{o}\right|+\sqrt{3} p\right) \frac{\sqrt{2} T_{o}}{3\left|V_{o}\right|-T_{o}}
\end{aligned}
$$

After the values of $C_{0}^{(1)}$ and $T_{0}^{(2)}$ are determined, the corresponding angles of internal friction need to be determined. This can be done by substituting the values of $C_{0}^{(1)}$ and $T_{0}^{(2)}$ into equations (39) and (40) to obtain $\mathrm{OB}$ and $\mathrm{OA}$ (Figure 17). With $\mathrm{OB}$ and $\mathrm{OA}$ known, the angles of internal friction can be found using equations (43) and (44). As a constraint, the angle of internal friction must be less than $90^{\circ}$; if the value of internal friction is greater than $90^{\circ}, C_{o}^{(1)}$ or $T_{o}^{(2)}$ must be adjusted so that $\phi<90^{\circ}$. Once the compression and extension friction angles are known, equations (63) and (64), which describe the failure surface presented in Figure 36, can be written as

$\frac{1}{V_{o}}\left[\frac{1-\sin \phi_{c}^{(1)}}{2 \sin \phi_{c}^{(1)}}\right] \sigma_{I}+\frac{1}{V_{o}}\left[\frac{\sin \phi_{c}^{(1)}-\sin \phi_{e}^{(1)}}{2 \sin \phi_{c}^{(1)} \sin \phi_{e}^{(1)}}\right] \sigma_{I I}-\frac{1}{V_{o}}\left[\frac{1+\sin \phi_{e}^{(1)}}{2 \sin \phi_{e}^{(1)}}\right] \sigma_{I I I}=1$

and 
$\frac{1}{V_{o}}\left[\frac{1-\sin \phi_{c}^{(2)}}{2 \sin \phi_{c}{ }^{(2)}}\right] \sigma_{I}+\frac{1}{V_{o}}\left[\frac{\sin \phi_{c}^{(2)}-\sin \phi_{e}{ }^{(2)}}{2 \sin \phi_{c}^{(2)} \sin \phi_{e}{ }^{(2)}}\right] \sigma_{I I}-\frac{1}{V_{o}}\left[\frac{1+\sin \phi_{e}{ }^{(2)}}{2 \sin \phi_{e}{ }^{(2)}}\right] \sigma_{I I I}=1$

with equation (74) describing the failure surface from $\theta=74.5^{\circ}$ to $90^{\circ}$ and equation (75) describing the failure surface from $\theta=30^{\circ}$ to $74.5^{\circ}$. From triaxial compression and extension testing of the Calcarenite specimens, $\phi_{c}{ }^{(2)}$ and $\phi_{e}{ }^{(1)}$ are known and the vertex was determined to be $V_{o}=-15.16 \mathrm{MPa}$. Using this vertex and the known relations to the deviator stress, the values of $C_{o}^{(1)}$ and $T_{o}^{(2)}$ can be determined using equations (72) and (73):

$$
\begin{gathered}
19.77=(\sqrt{3}(15.16 M P a+11.2 M P a)) \frac{\sqrt{2} C_{o}{ }^{(1)}}{45.48 M P a+C_{o}{ }^{(1)}} \\
C_{o}{ }^{(1)}=\frac{19.69 M P a}{.9812}=20.07 M P a \\
19.60=(\sqrt{3}(15.16 M P a+11.2 M P a)) \frac{\sqrt{2} T_{o}{ }^{(2)}}{45.48 M P a-T_{o}{ }^{(2)}} \\
T_{o}{ }^{(2)}=\frac{19.52 M P a}{1.84}=10.59 M P a
\end{gathered}
$$

The angle of internal friction associated with $C_{0}^{(1)}$ or $T_{0}^{(2)}$ can now be calculated from the values of $O A$ and $O B$ :

$$
\begin{aligned}
& O A=O V \tan \alpha=\frac{\sqrt{6}}{\frac{3}{C_{o}^{(1)}}+\frac{1}{V_{o}}}=\frac{\sqrt{6}}{\frac{3}{20.07 \mathrm{MPa}}+\frac{1}{15.16 \mathrm{MPa}}}=11.36 \mathrm{MPa} \\
& O B=O V \tan \beta=\frac{\sqrt{6}}{\frac{3}{T_{o}^{(2)}}-\frac{1}{V_{o}}}=\frac{\sqrt{6}}{\frac{3}{10.59 \mathrm{MPa}}-\frac{1}{15.16 \mathrm{MPa}}}=11.27 \mathrm{MPa}
\end{aligned}
$$

The angles of internal friction can be determined using equations (43) and (44):

$$
\begin{gathered}
11.36=\frac{2 \sqrt{6} V_{o} \sin \phi_{c}{ }^{(1)}}{3-\sin \phi_{c}{ }^{(1)}}=\frac{(74.27 M P a) \sin \phi_{c}{ }^{(1)}}{3-\sin \phi_{c}{ }^{(1)}} \\
\phi_{c}{ }^{(1)}=\sin ^{-1}\left(\frac{34.08 M P a}{62.91 M P a}\right)=23.45^{\circ}
\end{gathered}
$$




$$
\begin{gathered}
\text { 11.27 MPa }=\frac{2 \sqrt{6} V_{o} \sin \phi_{e}^{(2)}}{3+\sin \phi_{e}{ }^{(2)}}=\frac{(74.27 M P a) \sin \phi_{e}{ }^{(2)}}{3+\sin \phi_{e}{ }^{(2)}} \\
\phi_{e}{ }^{(2)}=\sin ^{-1}\left(\frac{33.81 M P a}{85.54 M P a}\right)=32.46^{\circ}
\end{gathered}
$$

Plugging the known values into equations (73) and (74), allows the equations of the planes to written as

$$
0.050 \sigma_{I}+0.01 \sigma_{I I}-0.126 \sigma_{I I I}=1
$$

and

$$
0.062 \sigma_{I}-0.034 \sigma_{I I}-0.094 \sigma_{I I I}=1
$$

2. The second approach assumes that each hexagon has its own vertex (Figure 38). Six constants are needed to define the failure surface; $C_{o}^{(1)}, C_{o}{ }^{(2)}, T_{o}^{(1)}, T_{o}^{(2)}, V_{o}^{(1)}$ and $V_{o}^{(2)}$. A convenient choice as to where the two vertices are located comes from the conventional triaxial compression and extension experiments. As shown in Figure 14, the original intercepts from the compression and extension best fit lines did not coincide. By taking these two intercepts as the vertices of the two hexagons a failure surface with two vertices can be formed. For the Calcarenite being tested, the vertex $V_{o}^{(1)}$ for $C_{o}^{(1)}$ and $T_{o}^{(1)}$ is $14.15 \mathrm{MPa}$ while the vertex $V_{o}^{(2)}$ for $C_{o}^{(2)}$ and $T_{o}^{(2)}$ is $15.93 \mathrm{MPa}$. 


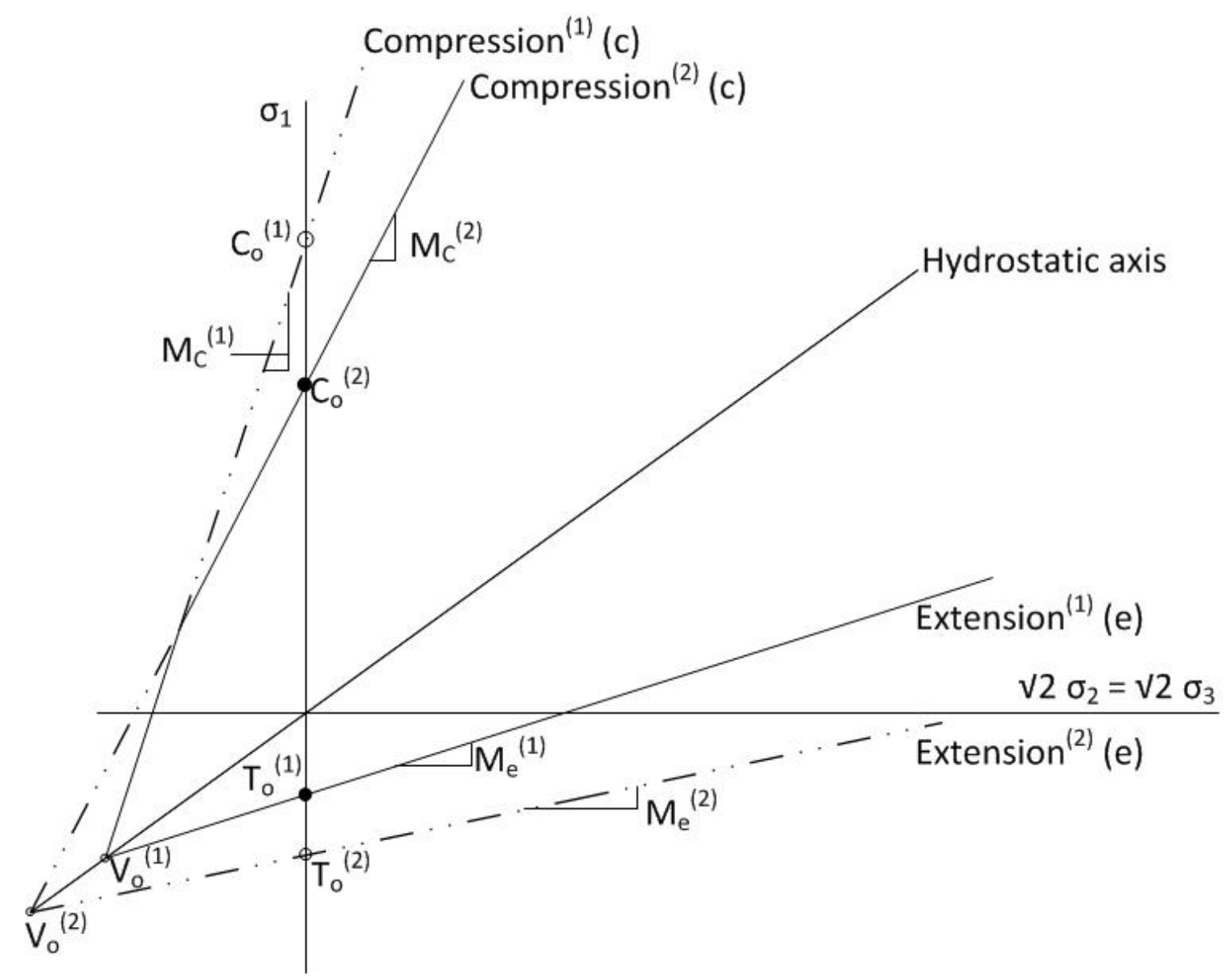

Figure 38. Cross-section of failure surface, developed assuming two vertices, along hydrostatic axis corresponding to $\sigma_{2}=\sigma_{3}$. Open circles are extrapolated from data.

The twelve-sided pyramidal failure surface in principal stress space with two vertices is shown in Figure 39. 


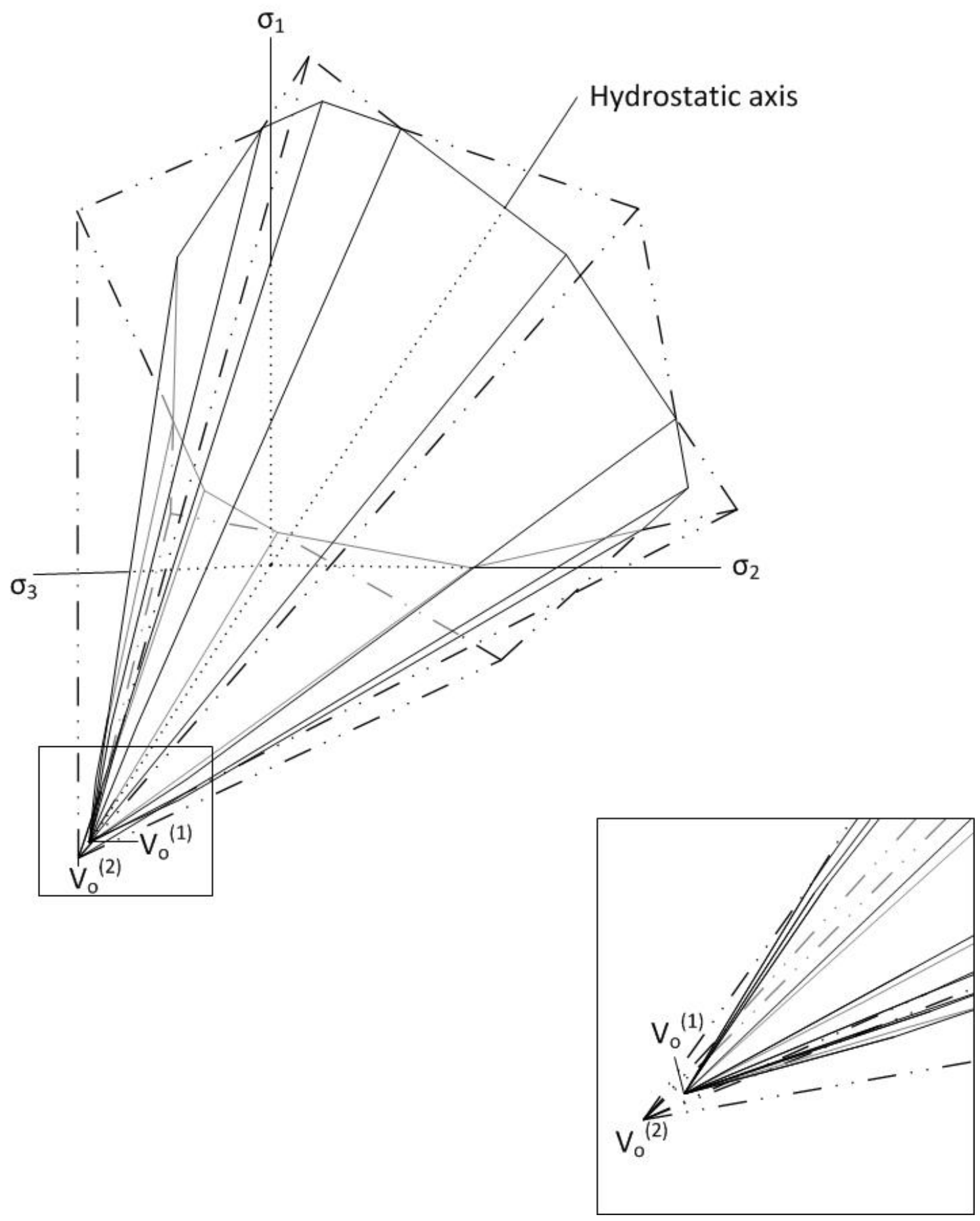

Figure 39. Twelve-sided pyramidal failure surface with two vertices. 
With the vertices known, the values of $C_{0}^{(1)}, C_{0}^{(2)}, T_{0}^{(1)}$ and $T_{0}^{(2)}$ can be determined using equation (72) and (73). Following the same steps as presented for a failure surface with one vertex, the angles of internal friction can be determined. Once all the angles of internal friction are determined, equations (63) and (64) can be written in terms of compression and extension friction angles and the vertices:

$$
\frac{1}{V_{o}^{(1)}}\left[\frac{1-\sin \phi_{c}^{(1)}}{2 \sin \phi_{c}^{(1)}}\right] \sigma_{I}+\frac{1}{V_{o}^{(1)}}\left[\frac{\sin \phi_{c}^{(1)}-\sin \phi_{e}^{(1)}}{2 \sin \phi_{c}^{(1)} \sin \phi_{e}^{(1)}}\right] \sigma_{I I}-\frac{1}{V_{o}^{(1)}}\left[\frac{1+\sin \phi_{e}^{(1)}}{2 \sin \phi_{e}^{(1)}}\right] \sigma_{I I I}=1
$$

and

$$
\frac{1}{V_{o}^{(2)}}\left[\frac{1-\sin \phi_{c}^{(2)}}{2 \sin \phi_{c}^{(2)}}\right] \sigma_{I}+\frac{1}{V_{o}^{(2)}}\left[\frac{\sin \phi_{c}^{(2)}-\sin \phi_{e}^{(2)}}{\sin \phi_{c}^{(2)} \sin \phi_{e}^{(2)}}\right] \sigma_{I I}-\frac{1}{V_{o}^{(2)}}\left[\frac{1+\sin \phi_{e}^{(2)}}{2 \sin \phi_{e}^{(2)}}\right] \sigma_{I I I}=1
$$

Again, considering $\mathrm{p}=11.2 \mathrm{MPa}$ and knowing the values of $V_{o}^{(1)}$ and $V_{o}^{(2)}$, the values of $C_{o}^{(1)}$, $C_{o}{ }^{(2)}, T_{o}^{(1)}$, and $T_{o}^{(2)}$ were determined to be $19.83 \mathrm{MPa}, 19.75 \mathrm{MPa}, 7.61 \mathrm{MPa}$, and 10.88 $\mathrm{MPa}$, respectively. Using equation (36), (37), (40), and (41) the angles of internal friction associated with these values were obtained: $\phi_{c}{ }^{(1)}=24.3^{\circ}, \phi_{c}{ }^{(2)}=19.8^{\circ}, \phi_{e}{ }^{(1)}=21.6^{\circ}$, and $\phi_{e}{ }^{(2)}=31.2^{\circ}$. By plugging these known values into equations (76) and (77), the failure surface equations can be written as

$$
0.050 \sigma_{I}+0.01 \sigma_{I I}-0.131 \sigma_{I I I}=1
$$

and

$$
0.062 \sigma_{I}-0.033 \sigma_{I I}-0.092 \sigma_{I I I}=1
$$

with equation (74) describing the failure surface from $\theta=74.5^{\circ}$ to $90^{\circ}$, and equation (75) describing the failure surface from $\theta=30^{\circ}$ to $74.5^{\circ}$. Similar to the six-sided pyramidal failure surface, the twelve-sided pyramidal failure surface can be constructed with a tension cut-off (Figure 40). The strength parameters of the three pyramidal surfaces developed (six-sided, twelve-sided with one vertex, and twelve-sided with two vertices) are presented in Table 9. 
Table 9. Material parameters determined from three different pyramidal failure surfaces.

\begin{tabular}{|c|c|c|c|c|c|c|c|c|c|c|}
\hline $\begin{array}{c}\text { Failure } \\
\text { surface }\end{array}$ & $\begin{array}{c}\phi_{\mathrm{c}}^{\left({ }^{(1)}\right.} \\
{\left[^{\circ}\right]}\end{array}$ & $\begin{array}{c}\phi_{\mathrm{e}}^{(1)} \\
{\left[{ }^{\circ}\right]}\end{array}$ & $\begin{array}{c}C_{o}^{(1)} \\
{[\mathrm{MPa}]}\end{array}$ & $\begin{array}{c}T_{0}^{(1)} \\
{[\mathrm{MPa}]}\end{array}$ & $\begin{array}{c}\left|V_{o}^{(1)}\right| \\
{[\mathrm{MPa}]}\end{array}$ & $\begin{array}{c}\phi_{\mathrm{c}}^{(2)} \\
{\left[^{\circ}\right]}\end{array}$ & $\begin{array}{c}\phi_{\mathrm{e}}^{(2)} \\
{\left[{ }^{\circ}\right]}\end{array}$ & $\begin{array}{c}C_{o}^{(2)} \\
{[\mathrm{MPa}]}\end{array}$ & $\begin{array}{c}T_{o}^{(2)} \\
{[\mathrm{MPa}]}\end{array}$ & $\begin{array}{c}\left|V_{o}^{(2)}\right| \\
{[\mathrm{MPa}]}\end{array}$ \\
\hline Six-sided & 20.2 & 21.0 & 16.10 & 7.91 & 15.16 & - & - & - & - & - \\
\hline $\begin{array}{c}\text { Twelve-sided } \\
\text { w/ one vertex }\end{array}$ & 23.4 & 21.0 & 20.07 & 7.91 & 15.16 & 20.2 & 32.5 & 16.10 & 10.59 & 15.16 \\
\hline $\begin{array}{c}\text { Twelve-sided } \\
\text { w/ two vertices }\end{array}$ & 24.3 & 21.6 & 19.83 & 7.61 & 14.15 & 19.8 & 31.2 & 19.75 & 10.88 & 15.93 \\
\hline
\end{tabular}

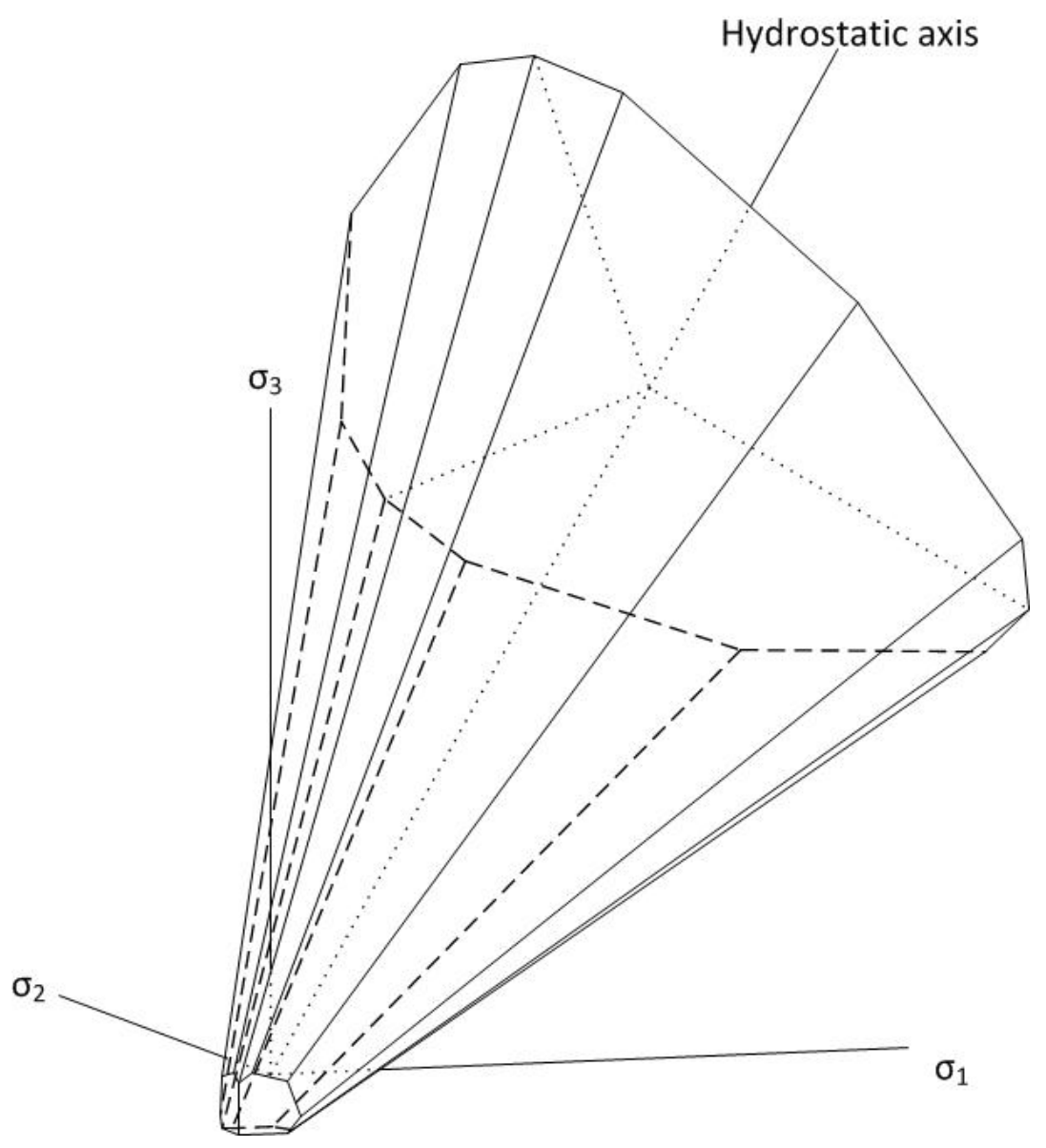

Figure 40. Twelve-sided failure surface with tension cut-offs. 


\subsection{Concluding remarks}

\subsection{Conclusions}

A series of experiments were performed on an isotropic Calcarenite sample to establish a linear failure criterion for rock that incorporates all three principal stresses. The linear failure criterion is described by three material parameters: the angles of internal friction in compression $\phi_{\mathrm{c}}$ and extension $\phi_{\mathrm{e}}$ as well as a common vertex $V_{o}$.

From conventional triaxial compression $\left(\sigma_{\|}=\sigma_{\| I}\right)$ and extension experiments $\left(\sigma_{l}=\sigma_{\|}\right)$performed with a Hoek-Franklin cell (or a similar testing apparatus), the intermediate stress can be evaluated and the angles of internal friction $\phi_{\mathrm{c}}$ and $\phi_{\mathrm{e}}$ can be determined. A convenient method to determine the values of $\phi_{\mathrm{c}}$ and $\phi_{\mathrm{e}}$ is to use the stress invariants, $p$ and $q$, where $p$ is the mean stress and $q$ is related to the second invariant of the stress deviator. However, the intersection of the compression and extension failure lines, on the $p$-axis, usually do not coincide. The calculated failure lines need to be adjusted (e.g. using a minimization of least-squares or the maximization of the coefficient of correlation $R^{2}$ ), with the constraint that the failure lines in compression and extension have the same intersection on the $p$-axis, known as the vertex $V_{o}$, which is the state of equal triaxial tension. After the adjustment, if angle of internal friction $\phi_{\mathrm{c}}$ and $\phi_{\mathrm{e}}$ are different, there is an intermediate stress effect. If $\phi_{\mathrm{c}}=\phi_{\mathrm{e}}$, it is still possible that an intermediate stress effect exists, but multi-axial testing is required to establish the relation.

Using the values of $V_{o}, \phi_{\mathrm{c}}$ and $\phi_{\mathrm{e}}$ determined from the adjusted $p-q$ diagram, a six-sided pyramidal surface that incorporates all three principal stresses can be constructed in principal stress space. The six sides of the failure surface can be described by a single equation:

$$
\frac{1}{V_{o}}\left[\frac{1-\sin \phi_{c}}{2 \sin \phi_{c}}\right] \sigma_{I}+\frac{1}{V_{o}}\left[\frac{\sin \phi_{c}-\sin \phi_{e}}{2 \sin \phi_{c} \sin \phi_{e}}\right] \sigma_{I I}-\frac{1}{V_{o}}\left[\frac{1+\sin \phi_{e}}{2 \sin \phi_{e}}\right] \sigma_{I I I}=1
$$

The advantage to this equation is that it contains the three principal stresses and allows for the description of the entire failure surface while knowing three material parameters, $V_{o}, \phi_{\mathrm{c}}$ and $\phi_{\mathrm{e}}$.

A uniaxial tension test was used to determine the tensile strength $T$, which allowed for a modification of the six-sided pyramidal surface. With the measured tensile strength, the six-sided pyramidal surface was modified to incorporate a tension cut-off. The tension cut-off is another pyramidal surface with three planes perpendicular to the principal stress axis at a value of $-T$ which intersects the six-sided pyramidal surface and acts as a limit to the tensile stress that can 
be applied to the material before failure will occur. The possible anisotropy in tensile strength was not investigated.

Another area of interest analyzed in this thesis was the intermediate stress effect at different stress states. In order to achieve a stress state where all three principal stresses were different $\left(\sigma_{1} \neq \sigma_{2} \neq \sigma_{3}\right)$, plane strain experiments were performed. By incorporating the data obtained during multi-axial experiments, an enhanced failure surface was developed. By examining the equipressure plane at a given mean stress, and by incorporating values from conventional triaxial compression and extension as well as a multi-axial experiments, it was determined that a twelvesided, piecewise linear failure surface would describe the material response better than the sixsided pyramidal surface. A twelve-sided pyramidal surface is a combination of two six-sided pyramidal surfaces, with the controlling pyramidal surface having the smaller deviator stress, at a particular stress state, which can be described using the Lode angle $\theta$.

To develop the twelve-sided pyramidal failure surface, two approaches can be used: (1) the two six-sided pyramidal failure surfaces have a common vertex; (2) the two six-sided pyramidal failure surfaces have independent vertices. It is important to determine the material parameters of each pyramidal surface, as once the material parameters are known, the equation for the six-sided surface can be used to describe the two pyramids that are associated with the twelve sided surface.

\subsection{Future work}

The suggested area of future work involves additional multi-axial testing, for both tensile and compressive normal stresses. Investigating the tensile strength of a material at different stress states would be appropriate to evaluate the tension cut-offs. In order to apply a tensile axial stress to a specimen under a confining stress, some slight modifications to the conventional triaxial testing apparatus would need to be made (Figure 41). Using the flexible cable method and the direct butt-jointing technique, a tensile stress could be applied to a specimen under confining stress. To avoid failure due to end effects, the specimen should have a reduced crosssection near its middle, but in order to apply a uniform confining stress the reduced cross-section would have to be filled in. A technique that could be used, would be to use a lathe to reduce the cross-sectional area of the specimen and then fill this reduced cross-sectional area in with hydrostone, labeled (5) in Figure 41. Another modification is to fabricate a stand to support the triaxial cell (6). 


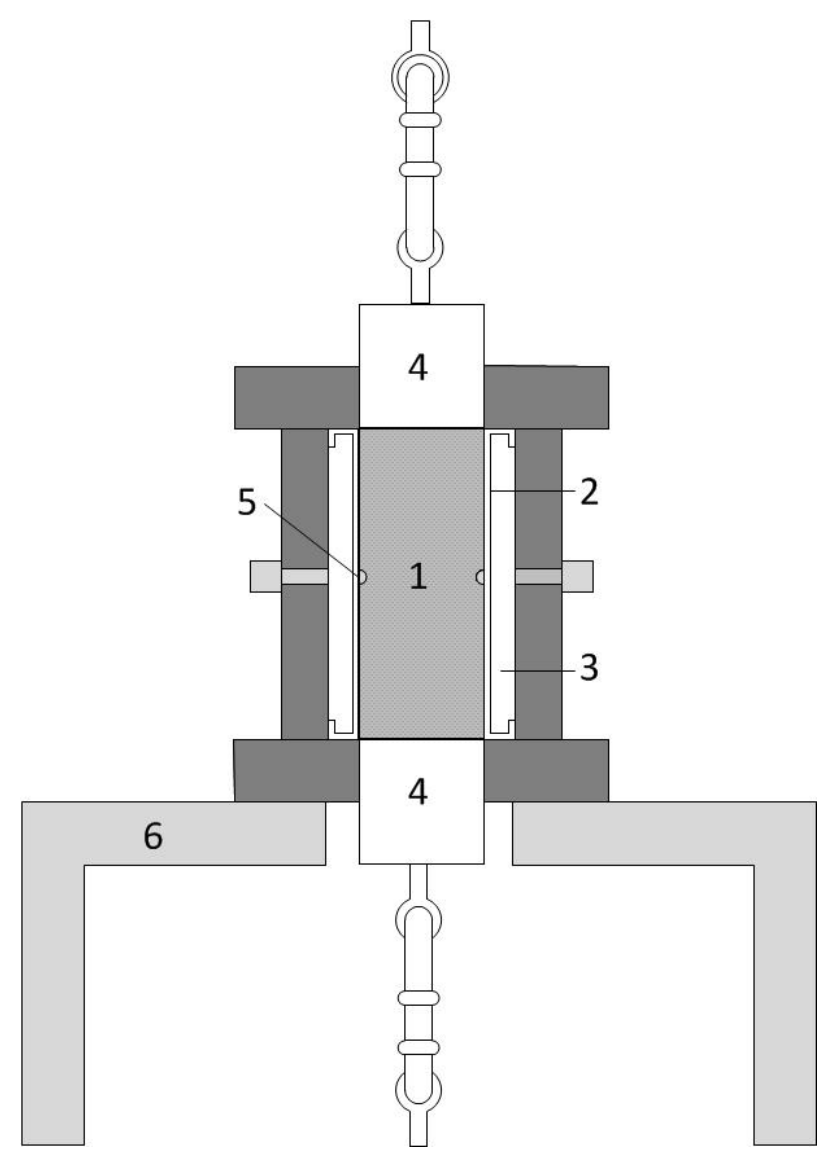

Figure 41. Modified conventional triaxial testing apparatus.

With this modified conventional triaxial apparatus, the predicted failure along the tension cut-off could be studied.

The multi-axial experiments were performed using the UMN plane-strain testing apparatus. An issue with this apparatus is that $\sigma_{\|}$cannot be controlled, which causes difficulties in developing specific stress states. A modification to the testing apparatus that would allow for $\sigma_{\|}$to be controlled can be made. By fabricating custom stainless steel flat jacks, labeled (5) in Figure 42, which would replace the flat and wedge, a specified value of $\sigma_{\|}$could be maintained. The recommended dimensions of the flat jacks would be $100.5 \mathrm{~mm}$ in height with a width of $44.0 \mathrm{~mm}$. The thickness of the flat jacks would be $4.0 \mathrm{~mm}$ with the capability of expanding to $4.30 \mathrm{~mm}$. It would be important to have bleeder valves on the flat jacks to ensure no air is in the system. 


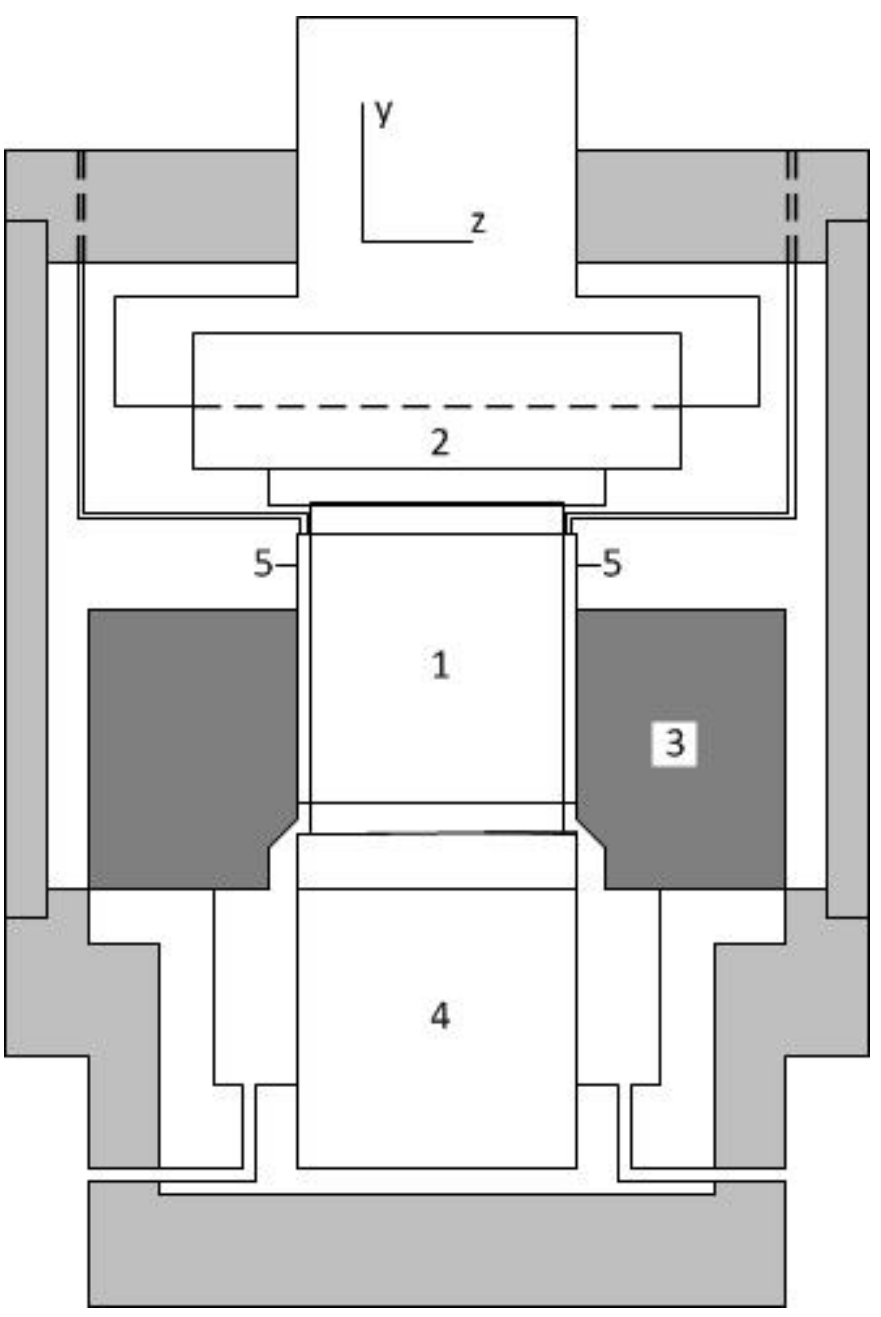

Figure 42. Modified UMN plane-strain apparatus. 


\subsection{References}

Borja, R. On the Numerical Integration of Three-invariant Elastoplastic Constitutive Models. Computer Methods in Applied Mechanics and Engineering 192.9-10 (2003): 1227-258.

Brown, E. T. Rock Characterization, Testing and Monitoring: ISRM Suggested Methods. New York: Published for the Commission on Testing Methods, International Society for Rock Mechanics by Pergamon, 1981.

Coulomb, C.A., In Memories de Mathematique et de Physique. Academie Royal des Sciences par divers sans, 7 (1773), pp. 343-382

Davis, R. O., and A. P. S. Selvadurai. Plasticity and Geomechanics. Cambridge, UK: Cambridge UP, 2002.

Dehler, W., and J. F. Labuz. Stress Path Testing of an Anisotropic Sandstone. Journal of Geotechnical and Geoenvironmental Engineering 133.1 (2007): 116.

Drucker, D. C. and Prager, W. (1952). Soil Mechanics and Plastic Analysis for Limit Design. Quarterly of Applied Mathematics, V. 10, N. 2 (1952), pp. 157-165.

Fairhurst, C. Laboratory Measurement of Some Physical Properties of Rock, in Proceedings of the $4^{\text {th }}$ Symposium on Rock Mechanics. (The Pennsylvania State University, November 1961), pp. 105-118. Bull. Mineral Industries Experiment Station, Pennsylvania State University, No. 76, (1961)

Franklin, John A., and Evert Hoeck. Developments in Triaxial Testing Technique. Rock Mechanics 2.4 (1970): 223-28.

Hawkes, I., and M. Mellor. Uniaxial Testing in Rock Mechanics Laboratories. Engineering Geology 4.3 (1970): 179-285.

Hoek, E., and E. T. Brown. Underground Excavations in Rock. London: Institution of Mining and Metallurgy, 1980.

Hoek E. and P. Marinos, A brief history of the development of the Hoek-Brown failure criterion. Soils and Rocks, No. 2 (2007)

Houlsby, G.T. A General Failure Criterion for Frictional and Cohesive Materials. Soils And Foundations 26.2 (1986): 97-101.

Jaeger, J. C., and N. G. W. Cook. Fundamentals of Rock Mechanics. London: Chapman and Hall, 1971.

Labuz, J., S. Dai, and E. Papamichos. Plane-strain Compression of Rock-like Materials. International Journal of Rock Mechanics and Mining Sciences \& Geomechanics Abstracts 33.6 (1996): 573-84.

Matsuoka, H. and Nakai, T. Stress-deformation and strength characteristics of soil under three different principal stresses, Proceedings of Japan Society of Civil Engineers, No. 232 (1974), pp. $59-70$. 
Mohr, O., Welche Umstande Bedingen die Elastizitatsgrenze und den Bruch eines Materials. Zeitschrift des Vereines Deutscher Ingenieure, 44 (1900), pp. 1524 - 1530.

Mogi, K. Effect of the Intermediate Principal Stress on Rock Failure. Journal of Geophysical Research 72.20 (1967): 5117.

Mogi, K. Fracture and Flow of Rocks under High Triaxial Compression. Journal of Geophysical Research 76.5 (1971): 1255-269.

Murrell, S. A. F. The Effect of Triaxial Stress Systems on the Strength of Rocks at Atmospheric Temperatures. Geophysical Journal International 10.3 (1965): 231-81.

Nadai, A. Theory of Flow and Fracture of Solids. New York, NY: McGraw-Hill, 1950.

Pariseau, W. Fitting Failure Criteria to Laboratory Strength Tests. International Journal of Rock Mechanics and Mining Sciences 44.4 (2007): 637-46.

Paul, B., A modification of the Coulomb-Mohr Theory of Fracture. Journal of Applied Mechanics 28 (1961), $259-268$.

Paul, B., Macroscopic Criteria for Plastic Flow and Brittle Fracture, Treatise on Fracture (ed. $\mathrm{H}$. Liebowitz), II (1968a), pp. 313 - 496. Academic Press, New York.

Paul, B., A three-dimensional stress Fracture Criterion based on Microscopic Flaws, IngersollRand Research, Inc. (1968b), Princeton, N. J.

Paul, B. Generalized Pyramidal Fracture and Yield Criteria. International Journal of Solids and Structures 4.2 (1968c): 175-96.

Pincus HJ. Closed-form/least-squares failure envelopes for rock strength. International Journal of Rock Mechanics and Mining Sciences and Geomechanics 37 (2000): 763-785

Wawersik, W., L. Carlson, D. Holcomb, and R. Williams. New Method for True-triaxial Rock Testing. International Journal of Rock Mechanics and Mining Sciences 34.3-4 (1997) 